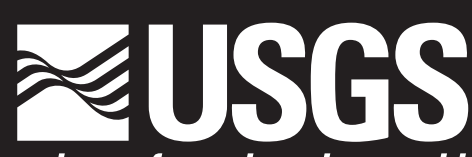

science for a changing world

Prepared in cooperation with the Minnesota Pollution Control Agency and St. Cloud State University

\title{
Alkylphenols, Other Endocrine-Active Chemicals, and Fish Responses in Three Streams in Minnesota- Study Design and Data, February-September 2007
}
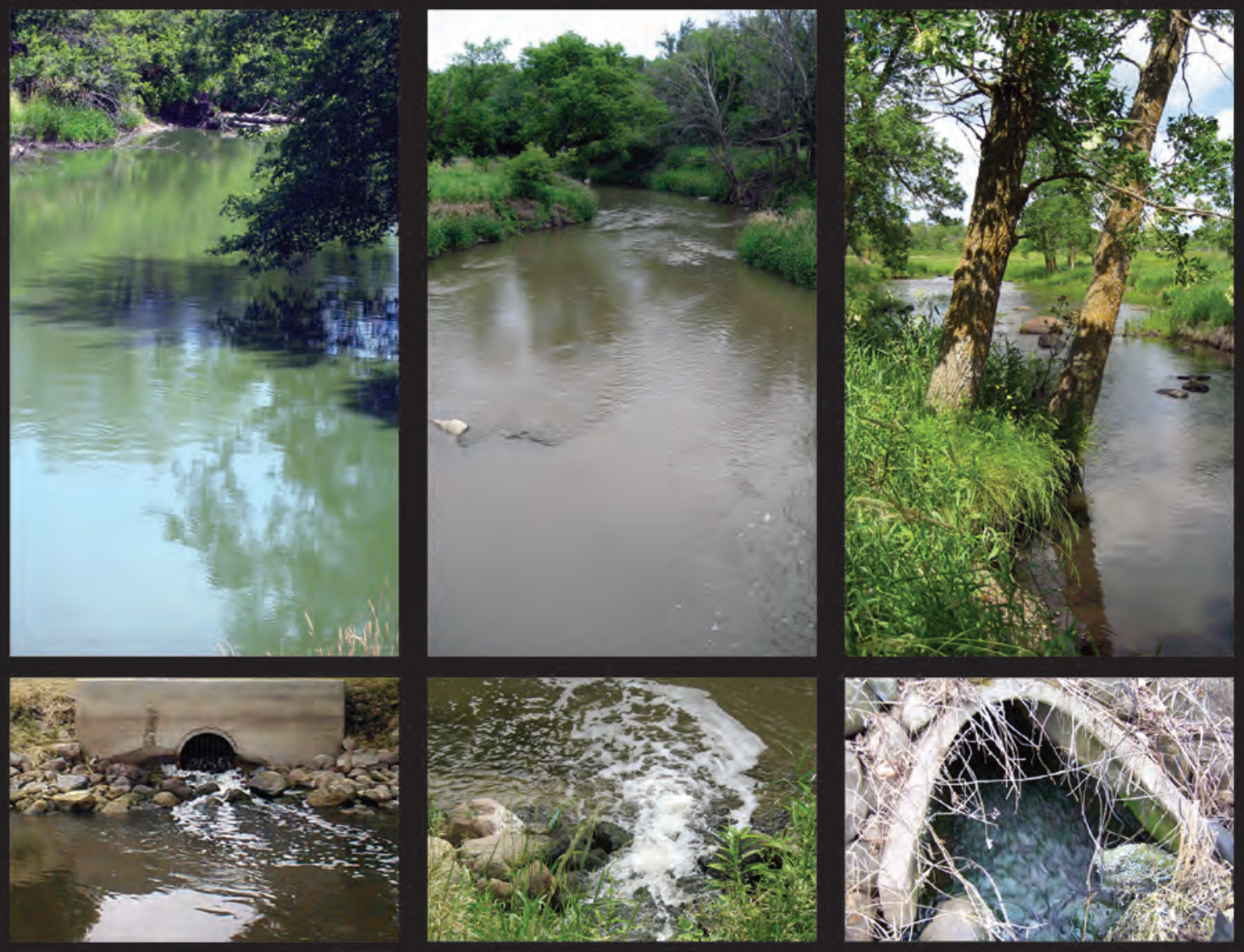

Data Series 405 
Cover photograph. Left top: South Fork Crow River near Hutchinson, Minn., 1999 (photograph by Kathy Lee, U.S. Geological Survey). Left bottom: Hutchinson, Minn., wastewater-treatment plant discharge, 1999 (photograph by Phil Talmage, U.S. Geological Survey). Center top: Redwood River near Marshall, Minn., 1999 (photograph by Kathy Lee, U.S. Geological Survey). Center bottom: Marshall, Minn., wastewater-treatment plant discharge, 1999 (photograph by Kathy Lee, U.S. Geological Survey). Right top: Grindstone River near Hinckley, Minn., 2007 (photograph by John Greene, U.S. Geological Survey). Right bottom: Hinckley, Minn., wastewater-treatment plant discharge, 2007 (photograph by John Greene, U.S. Geological Survey). 


\section{Alkylphenols, Other Endocrine-Active Chemicals, and Fish Responses in Three Streams in Minnesota-Study Design and Data, February-September 2007}

By Kathy E. Lee, Heiko L. Schoenfuss, Nathan D. Jahns, Greg K. Brown, and Larry B. Barber

Prepared in cooperation with the Minnesota Pollution Control Agency and St. Cloud State University

Data Series 405 


\title{
U.S. Department of the Interior DIRK KEMPTHORNE, Secretary
}

\author{
U.S. Geological Survey \\ Mark D. Myers, Director
}

U.S. Geological Survey, Reston, Virginia: 2008

This and other USGS information products are available at http://store.usgs.gov/
U.S. Geological Survey
Box 25286, Denver Federal Center
Denver, C0 80225
To learn more about the USGS and its information products visit http://www.usgs.gov/
1-888-ASK-USGS

Any use of trade, product, or firm names is for descriptive purposes only and does not imply endorsement by the U.S. Government.

Although this report is in the public domain, permission must be secured from the individual copyright owners to reproduce any copyrighted materials contained within this report.

Suggested citation:

Lee, K.E., Schoenfuss, H.L., Jahns, N.D., Brown, G.K., and Barber, L.B., 2008, Alkylphenols, other endocrine-active chemicals, and fish responses in three streams in Minnesota-Study design and data, February-September 2007: U.S. Geological Survey Data Series 405, 44 p. plus appendixes. 


\section{Contents}

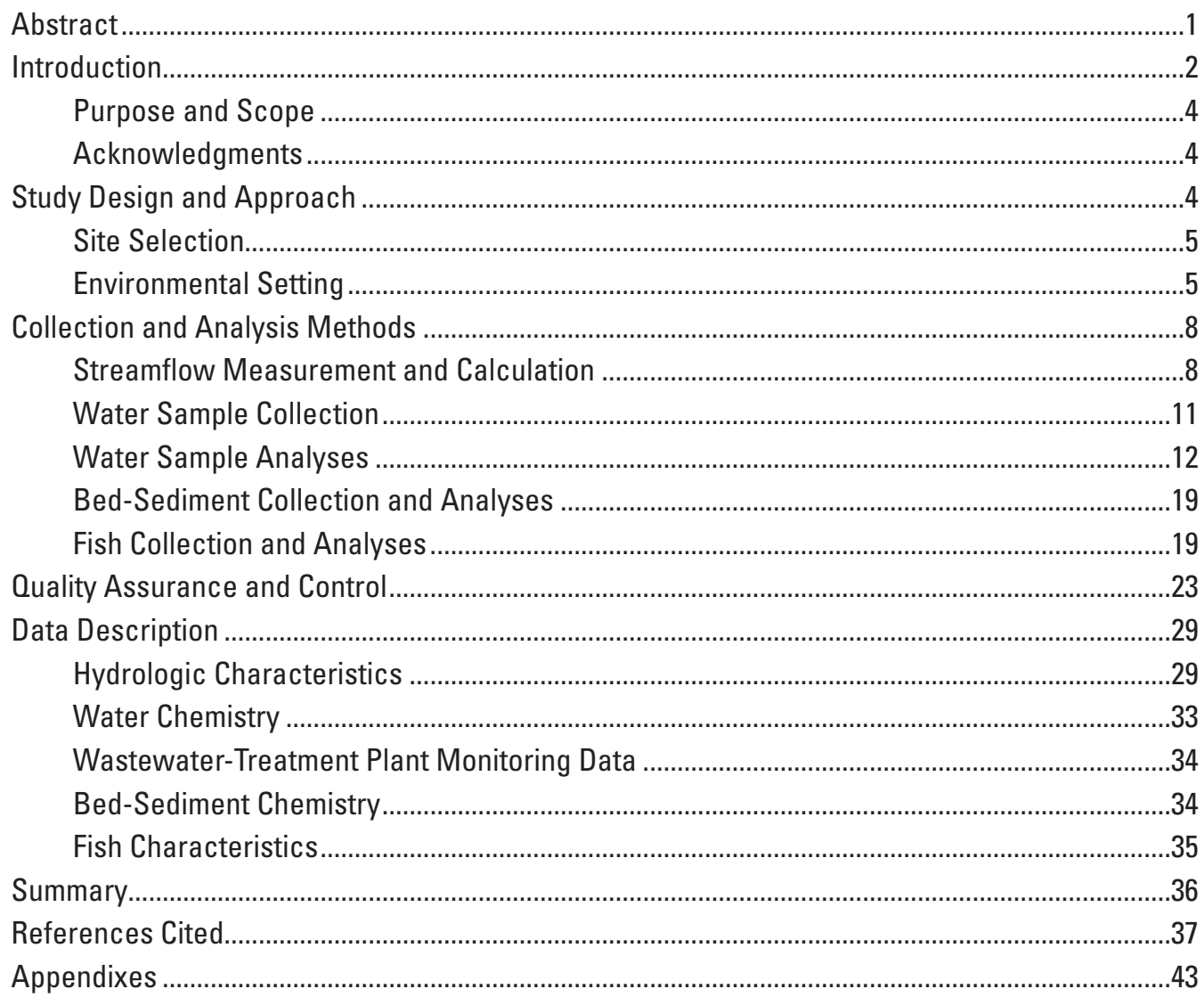

Appendix 1. Concentrations of organic chemicals in water analyzed at U.S.

Geological Survey National Research Program Laboratory........................................43

Appendix 2. Concentrations of organic chemicals in water samples analyzed using Laboratory Schedule 4433 at the U.S. Geological Survey National Water Quality Laboratory.

Appendix 3. Concentrations of nutrients in water samples analyzed at U.S. Geological Survey National Water Quality Laboratory.....

Appendix 4. Dissolved concentrations of major ions in water samples analyzed at the U.S. Geological Survey National Water Quality Laboratory ...............................43

Appendix 5. Concentrations of organic chemicals analyzed in bed-sediment samples at the U.S. Geological Survey National Research Program Laboratory ........................43

Appendix 6. Hydrologic characteristics of sampling sites ..................................................44

Appendix 7. Physical measurements of water quality ............................................................44

Appendix 8. Concentrations of total alkylphenols and atrazine in water analyzed at U.S. Geological Survey National Research Program Laboratory .................................44

Appendix 9. Chemical characteristics of wastewater-treatment plant effluents studied.

Appendix 10. Characteristics of fish collected and analyzed by St. Cloud State University 


\section{Figures}

1. Map showing location of wastewater-treatment plants that discharge to surface waters in Minnesota..

2. Schematic diagram showing relative locations of sampling sites.

3-5. Maps showing-

3. South Fork Crow River drainage basin, wastewater-treatment plants, and sampling sites along the sampling reach

4. Redwood River drainage basin, wastewater-treatment plants, and sampling sites along the sampling reach

5. Grindstone River drainage basin, wastewater-treatment plants, and sampling sites along the sampling reach

6-12. Photographs showing-

6. Streamflow measurements at the Grindstone River, September 2007, and the South Fork Crow River under ice, February 2007.

7. Water collection at the Redwood River near Marshall, Minnesota, February 2007

8. Collection bottles inside the auto sampler used to collect samples at the Redwood River near Marshall, Minnesota, 2007

9. Bed-sediment sample collection and processing at the Grindstone River, June 2007

10. Fish collection using electrofishing techniques.....................................................20

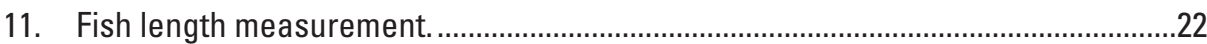

12. Histological cassette containing testes and liver tissue

13-15. Graphs showing-

13. Hydrologic characteristics of precipitation, continuous streamflow at nearby streamflow gaging stations, and instantaneous streamflow measured at the time of sampling for the South Fork Crow River...

14. Hydrologic characteristics of precipitation, continuous streamflow at nearby streamflow gaging stations, and instantaneous streamflow measured at the time of sampling for the Redwood River

15. Hydrologic characteristics of precipitation, continuous streamflow at nearby streamflow gaging stations, and instantaneous streamflow measured at the time of sampling for the Grindstone River 


\section{Tables}

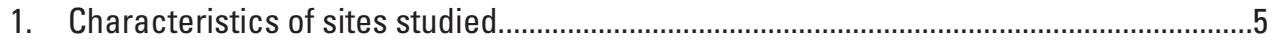

2. Land-use characteristics of drainage basins studied.......................................................

3. Characteristics of wastewater-treatment plants studied.................................................

4. Physical properties and chemicals analyzed for in water samples ................................13

5. Chemicals for which bed-sediment samples were analyzed at the U.S.

Geological Survey National Research Program Laboratory ............................................21

6. Numbers of fish collected by St. Cloud State University .................................................22

7. Summary of chemicals detected in field blank samples ...............................................25

8. Concentrations and detections of chemicals for which environmental and replicate samples were analyzed ...............................................................................26

\section{Conversion Factors and Datums}

SI to inch/pound (used for analytical measures and instruments)

\begin{tabular}{lll}
\hline \multicolumn{1}{c}{ Multiply } & By & \multicolumn{1}{c}{ To obtain } \\
\hline kilometer $(\mathrm{km})$ & Length & \\
meter $(\mathrm{m})$ & 0.6214 & mile $(\mathrm{mi})$ \\
\hline & 3.21 & foot $(\mathrm{ft})$ \\
\hline liter $(\mathrm{L})$ & Volume & \\
milliliter $(\mathrm{mL})$ & 0.2642 & gallon (gal) \\
cubic meter $\left(\mathrm{m}^{3}\right)$ & 0.0338 & ounce, fluid (fl. oz) \\
cubic meter $\left(\mathrm{m}^{3}\right)$ & 0.0002642 & million gallons $(\mathrm{Mgal})$ \\
& 35.31 & cubic foot $\left(\mathrm{ft}^{3}\right)$ \\
\hline centimeter per second $(\mathrm{cm} / \mathrm{s})$ & Flow rate & \\
\hline & 0.3937 & inch per second (in/s) \\
\hline gram $(\mathrm{g})$ & Mass & \\
\hline & 0.03527 & ounce, avoirdupois $(\mathrm{oz})$ \\
\hline kilopascal $(\mathrm{kPa})$ & Pressure & \\
\hline
\end{tabular}


Inch/Pound to SI

\begin{tabular}{lcl}
\hline \multicolumn{1}{c}{ Multiply } & By & \multicolumn{1}{c}{ To obtain } \\
\hline inch (in.) & Length & \\
foot (ft) & 2.54 & centimeter $(\mathrm{cm})$ \\
mile (mi) & 0.3048 & meter $(\mathrm{m})$ \\
& 1.609 & kilometer $(\mathrm{km})$ \\
\hline square mile $\left(\mathrm{mi}^{2}\right)$ & Area & \\
square mile $\left(\mathrm{mi}^{2}\right)$ & 259.0 & hectare $(\mathrm{ha})$ \\
& 2.590 & square kilometer $\left(\mathrm{km}^{2}\right)$ \\
\hline gallon $($ gal) & Volume & \\
million gallons $(\mathrm{Mgal})$ & 3.785 & liter $(\mathrm{L})$ \\
cubic foot $\left(\mathrm{ft}^{3}\right)$ & 3,785 & cubic meter $\left(\mathrm{m}^{3}\right)$ \\
& 0.02832 & cubic meter $\left(\mathrm{m}^{3}\right)$ \\
\hline gallon per day (gal/d) & Flow rate & \\
million gallons per day $(\mathrm{Mgal} / \mathrm{d})$ & 0.003785 & cubic meter per day $\left(\mathrm{m}^{3} / \mathrm{d}\right)$ \\
\hline
\end{tabular}

Temperature in degrees Celsius $\left({ }^{\circ} \mathrm{C}\right)$ may be converted to degrees Fahrenheit $\left({ }^{\circ} \mathrm{F}\right)$ as follows:

$$
{ }^{\circ} \mathrm{F}=\left(1.8 \times^{\circ} \mathrm{C}\right)+32
$$

Temperature in degrees Fahrenheit $\left({ }^{\circ} \mathrm{F}\right)$ may be converted to degrees Celsius $\left({ }^{\circ} \mathrm{C}\right)$ as follows:

$$
{ }^{\circ} \mathrm{C}=\left({ }^{\circ} \mathrm{F}-32\right) / 1.8
$$

Vertical coordinate information is referenced to the North American Vertical Datum of 1988 (NAVD 88).

Horizontal coordinate information is referenced to the North American Datum of 1983 (NAD 83).

Altitude, as used in this report, refers to distance above the vertical datum.

Specific conductance is given in microsiemens per centimeter at 25 degrees Celsius $(\mu \mathrm{S} / \mathrm{cm}$ at $\left.25^{\circ} \mathrm{C}\right)$.

Concentrations of chemical constituents in water are given in milligrams per liter (mg/L), micrograms per liter $(\mu \mathrm{g} / \mathrm{L})$, or nanograms per liter (ng/L). Concentrations of chemical constituents in bed sediment are given in nanograms per gram $(\mathrm{ng} / \mathrm{g})$. 


\section{Abbreviations and Acronyms}

\begin{tabular}{|c|c|}
\hline$\mu \mathrm{L}$ & microliter \\
\hline$\mu \mathrm{m}$ & micrometer \\
\hline amu & atomic mass units \\
\hline $\mathrm{ng} / \mathrm{g}$ & nanograms per gram \\
\hline $\mathrm{ng} / \mu \mathrm{L}$ & nanogram per microliter \\
\hline $\mathrm{nm}$ & nanometer \\
\hline AHTN & acetyl-hexamethyl-tetrahydronaphthalene \\
\hline AP & alkylphenol \\
\hline APEC & alkylphenol ethoxycarboxylate \\
\hline AP1E0 & alkylphenol monoethoxylate \\
\hline APEO & alkylphenol polyethoxylate \\
\hline BHA & 3-tert-butyl-4-hydoxyanisole \\
\hline CBOD5 & 5-day carbonaceous biochemical oxygen demand \\
\hline CCV & continuing calibration verification solution \\
\hline CLLE & continuous liquid-liquid extraction \\
\hline DEET & N,N-diethyl-meta-toluamide \\
\hline DO & dissolved oxygen \\
\hline DOC & dissolved organic carbon \\
\hline EAC & endocrine-active chemical \\
\hline EDTA & ethylenediaminetetraacetic acid \\
\hline ELISA & enzyme linked immunosorbent assay \\
\hline GSI & gonadosomatic index \\
\hline НHCB & hexahydrohexamethyl-cyclopenta-benzopyran \\
\hline HP & Hewlett Packard \\
\hline $\mathrm{HSI}$ & hepatosomatic index \\
\hline MDL & method detection limit \\
\hline MRL & minimum reporting level \\
\hline $\mathrm{n}$ & number sampled \\
\hline NP & 4-nonylphenol \\
\hline NP1EC & nonylphenolmonoethoxycarboxylate \\
\hline NP2EC & nonylphenoldiethoxycarboxylate \\
\hline NP3EC & nonlyphenoltriethoxycarboxylate \\
\hline
\end{tabular}




\begin{tabular}{|c|c|}
\hline NP4EC & nonlyphenoltetraethoxycarboxylate \\
\hline NPEC & nonylphenol ethoxycarboxylate \\
\hline NP1EO & 4-nonylphenol monoethoxylate \\
\hline NP2EO & 4-nonylphenol diethoxylate \\
\hline NPEO & nonylphenolpolyethoxylate \\
\hline NRP & National Research Program Laboratory \\
\hline NTA & nitrilotriacetic acid \\
\hline NWQL & National Water Quality Laboratory \\
\hline $\mathrm{OP}$ & octylphenol \\
\hline OP1EO & 4-octylphenol monoethoxylate \\
\hline OP2EO & 4-octylphenol diethoxylate \\
\hline OPEO & octylphenol polyethoxylate \\
\hline $\mathrm{R}^{2}$ & coefficient of determination \\
\hline RPD & relative percent difference \\
\hline SUVA $_{254}$ & specific ultraviolet absorbance at wavelength of 254 nanometers \\
\hline TOC & total organic carbon \\
\hline TOP & 4-tert-octylphenol \\
\hline USGS & U.S. Geological Survey \\
\hline UV & ultraviolet \\
\hline $\mathrm{UV}_{254}$ & ultraviolet absorbance at a wavelength of 254 nanometers \\
\hline $\operatorname{Vtg}$ & vitellogenin \\
\hline WWTP & wastewater-treatment plant \\
\hline
\end{tabular}




\title{
Alkylphenols, Other Endocrine-Active Chemicals, and Fish Responses in Three Streams in Minnesota-Study Design and Data, February-September 2007
}

\author{
By Kathy E. Lee', Heiko L. Schoenfuss ${ }^{2}$, Nathan D. Jahns², Greg Brown², and Larry Barber ${ }^{1}$
}

\section{Abstract}

This report presents the study design and environmental data for an integrated chemical and biological study of three streams (South Fork Crow River, Redwood River, and Grindstone River) that receive wastewater in Minnesota. The objective of the study was to identify distribution patterns of endocrine-active chemicals and other organic chemicals indicative of wastewater, and to identify fish responses in the same streams. Endocrine-active chemicals are a class of chemicals that interfere with the natural regulation of endocrine systems, and an understanding of their distribution in aquatic systems is important so that aquatic organism exposure can be evaluated.

This study was a cooperative effort of the U.S. Geological Survey (USGS), the Minnesota Pollution Control Agency, and St. Cloud State University (St. Cloud, Minn.). The USGS collected and analyzed water and quality-assurance samples and measured streamflow during six sampling events in each of three streams. Water samples were collected upstream from and at two successive points downstream from wastewatertreatment plant (WWTP) effluent discharge and from treated effluent from February through September 2007. Bedsediment samples were collected during one sampling period at each of the stream locations. Water and bed-sediment samples were analyzed for endocrine-active chemicals including alkylphenols, alkylphenol polyethoxylates, and nonylphenol ethoxycarboxlylates (NPECs). Water samples also were analyzed for major ions, nutrients, and organic carbon. In addition, as part of an intensive time-series investigation, the USGS staff collected daily water samples for 8 weeks from the Redwood River near Marshall, Minn., for analyses of total alkylphenols and atrazine. St. Cloud State University staff collected and analyzed fish to determine male fish responses at all water sampling sites and at an additional site near the discharge of wastewater-treatment plant effluent to these streams. Male fish responses included the presence and concentration of vitellogenin in plasma, gonadosomatic indices, and histological characterizations of liver and testes tissue.

\footnotetext{
${ }^{1}$ U.S. Geological Survey.

${ }^{2}$ St. Cloud State University, St. Cloud, Minn.
}

Hydrologic, chemical and biological characteristics were different among sites. The percentage of streamflow contributed by WWTP effluent (ranging from less than 1 to 79 percent) was greatest at the South Fork Crow River and least at the Grindstone River. WWTP effluent generally contributed the greatest percentage of streamflow during winter and late summer when streamflows were low.

A wide variety of chemicals were detected. More chemicals were detected in WWTP effluent samples than in stream samples during most time periods. The most commonly detected chemicals in samples collected monthly and analyzed at the USGS National Research Program Laboratory were 2,6-di-tert-butyl-1,4-benzoquinone, 2,6-di-tertbutyl-4-methylphenol, 3-beta-coprostanol, 4-methylphenol, 4-nonylphenol (NP), 4-tert-octylphenol, bisphenol A, cholesterol, ethylenediaminetetraacetic acid, and triclosan.

The chemicals 4-nonylphenolmonoethoxycarboxylate (NP1EC), 4-nonylphenoldiethoxycarboxylate (NP2EC), and 4-nonylphenoltriethoxycarboxylate (NP3EC) also were detected. Excluding nondetections, the sum of NP1EC through NP3EC concentrations ranged from 5.1 to $260 \mu \mathrm{g} / \mathrm{L}$ among all samples.

NP was detected in upstream, effluent, and downstream samples in each stream during at least one time period. NP was detected in 49 percent of environmental samples. Excluding nondetections, concentrations of NP ranged from 100 to 880 nanograms per liter among all samples. NP was also detected in more than one-half of the bed-sediment samples.

The most commonly detected wastewater indicator chemicals in samples analyzed by schedule 4433 at the USGS National Water Quality Laboratory were 3,4-dichlorophenyl isocyanate, acetyl-hexamethyl-tetrahydronaphthalene, benzophenone, cholesterol, hexahydrohexamethylcyclopenta-benzopyran, N,N-diethyl-meta-toluamide, and tri(dichloroisopropyl) phosphate.

Male fish responses were the focus of the fish analyses, and 508 male fish were collected among all sampling sites. Vitellogenin was detected in 57 percent of the 415 male fish analyzed; concentrations ranged from an estimated value of 0.1 to 330 micrograms per milliliter $(\mu \mathrm{g} / \mathrm{mL})$ (average of $17.1 \mu \mathrm{g} / \mathrm{mL}$ ). Intersex (the presence of oocytes in testes tissue) 
was observed in only one fathead minnow from an upstream site on the Grindstone River.

\section{Introduction}

Endocrine-active chemicals (EACs) have been identified in wastewater-treatment plant effluents and surface waters worldwide (Ahel and others, 1994a, b; Desbrow and others, 1998; Ternes, 1998; Kolpin and others, 2002), and more specifically in Minnesota (Barber and others, 2000, 2007; Lee and others, 2004; Martinovic and others, 2008). EACs are a class of chemicals that interfere with the natural regulation of fish endocrine systems and may mimic or block the function of natural hormones (Kime, 1998; National Research Council, 1999). This interference commonly is referred to as endocrine disruption. Although factors contributing to endocrine disruption are complex, laboratory studies have confirmed that certain classes of chemicals, such as hormones, alkylphenols (APs), plastic components, and pesticides, affect the endocrine systems of fish through biochemical, structural, and behavioral disruption (Jobling and Sumpter, 1993; Jobling and others, 1996; Ankley and others, 1998; Kime, 1998; Miles-Richardson and others, 1999; Harries and others, 2000; Bistodeau and others, 2006; Barber and others, 2007; Schoenfuss and others, 2008).

Wastewater-treatment plant (WWTP) effluent has been identified as a major source or pathway by which EACs travel to the aquatic environment (Ahel and others, 1994a, b; Desbrow and others, 1998; Ternes and others, 1999; Barber and others, 2000; Johnson and Sumpter, 2001; Lee and others, 2004; Martinovic and others, 2008). The almost ubiquitous presence of the APs and alkylphenol polyethoxylates (APEOs) in North American surface waters (Kolpin and others, 2002) indicates a potential role in causing endocrine disruption in exposed fish despite the fact that hormones such as 17ßestradiol are generally more potent as endocrine disruptors (as much as 100,000 times) than most EACs (Thorpe and others, 2001). APs are widely used surfactants (Talmage, 1994) and commonly are detected in WWTP effluent at microgramper-liter concentrations (Giger and others, 1981; Ahel and others, 1994a; Barber and others, 2000). Following wastewater treatment, APs are discharged to receiving waters in the form of 4-nonylphenol (NP), octylphenol (OP), nonylphenol polyethoxylates (NPEOs), octylphenol polyethoxylates (OPEOs), and nonylphenol ethoxycarboxylates (NPECs) (Giger and others, 1987; Naylor and others, 1992; Ahel and others, 1994b). The degradation of APEOs in the environment can result in the formation of APs and alkylphenol ethoxycarboxylates (APECs). Of particular concern is the degradation of APEOs within sewage treatment works into the shorter chained APs such as NP (Giger and others, 1981), which are more estrogenic (Jobling and others, 1996) than APEOs. The U.S. Environmental Protection Agency established final chronic aquatic-life criteria in streams for the protection of aquatic life for NP based on a 4-day average concentration not to exceed 6.6 micrograms per liter $(\mu \mathrm{g} / \mathrm{L})$ in lakes and streams for the protection of aquatic life (U.S. Environmental Protection Agency, 2005a).

In Minnesota, several studies have found that WWTP effluent can be a major source or pathway by which EACs travel to surface waters (Barber and others, 2000, 2007; Lee and others, 2004; Matinovic and others, 2008). Lee and others (2004) found that APs and APEOs composed the dominant EAC class in four WWTPs studied in Minnesota, and that $\mathrm{AP}$ and APEO concentrations varied temporally within any one WWTP as well as among WWTPs. Specifically, in the Minneapolis-St. Paul Metropolitan WWTP effluent, which is discharged to the Mississippi River, NP concentrations ranged from 0.9 to $2.3 \mu \mathrm{g} / \mathrm{L}$, and the sum of NP, 4-nonylphenol monoethoxylate (NP1EO), 4-nonylphenol diethoxylate (NP2EO), and total NPEC concentrations were as much as $150 \mu \mathrm{g} / \mathrm{L}$ (Barber and others, 2000; Lee and others, 2004). Lee and others $(2004,2008)$ also found numerous wastewater indicator chemicals such as cholesterol, caffeine, N,N-diethyl-metatoluamide (DEET), and EACs (3-tert-butyl-4-hydoxyanisole (BHA), 4-cumylphenol, acetyl-hexamethyl-tetrahydronapthalene (AHTN), hexahydrohexamethyl-cyclopenta-benzopyran (HHCB), benzo[a]pyrene, beta-sitosterol, and bisphenol-A) in WWTP effluent or surface waters in Minnesota. Exposure of aquatic organisms to EACs is likely on the basis of results of these studies and WWTP distribution in Minnesota (Barber and others, 2000; Lee and others, 2004; Martinovic and others, 2008).

In Minnesota, indications of endocrine disruption in male carp (Cyprinus carpio) and in male walleye (Stizostedion vitreum) have been documented in the effluent channel of the Minneapolis-St. Paul Metropolitan WWTP (Folmar and others, 1996, 2001; Lee and others, 2000) and in streams throughout Minnesota (Lee and others, 2000, 2008; Lee and Blazer, 2005). Vitellogenin (Vtg; an egg yolk protein normally present in female fish) in male fish plasma, and intersex occurrence (oocytes present in testes tissue) in male fish are commonly used indicators of endocrine disruption. Vtg was detected in male common carp (cyprinus carpio) in five Minnesota streams directly downstream from wastewater effluent and in four streams not directly downstream from wastewatertreatment-plant effluent (Lee and others, 2000). Intersex characteristics were documented in smallmouth bass (Micropterus dolomieu) at eight sites along the Mississippi River from the upper reaches of the River near Brainerd, Minn., downstream to Lake Pepin (Lee and Blazer, 2005). More recently, Lee and others (2008) documented Vtg in male fish collected from selected sites along the Mississippi River reach from the headwaters near Lake Itasca to Brownsville, Minn., at the Minnesota-Iowa State boundary.

Current research regarding the fate and occurrence of EACs and endocrine disruption has been focused on point sources of EACs such as WWTP and industrial effluent. However, other potential sources include nonpoint-source runoff and septic systems. Runoff from agricultural land may contain 
APs because they are used in pesticide formulations as wetting and spreading agents (U.S. Environmental Protection Agency, 2006a). Lee and others (2000) found indications of endocrine disruption in small streams draining agricultural land with no obvious source of WWTP effluent, which indicated an upstream source such as septic system discharge or nonpoint sources of EACs.

Many of the investigations into EACs and their associated effects are focused on large municipal WWTPs (Folmar and others, 1996, 2001; Barber and others, 2007; Martinovic and others, 2008; Vajda and others, 2008) and large river systems (Lee and others, 2008). Contributions from smaller WWTPs often are overlooked even though small WWTPs with flows less than 1 million gallons per day $(\mathrm{Mgal} / \mathrm{d})$ are more common in a given area than large WWTPs with flows greater than
$1 \mathrm{Mgal} /$ day. For example, more than 500 WWTPs in Minnesota have an average design flow of less than or equal to $1 \mathrm{Mgal} / \mathrm{d}$, but only about $60 \mathrm{WWTPs}$ are considered major dischargers (fig. 1) (Minnesota Pollution Control Agency, oral and written commun., July 14, 2006).

Although previous studies have indicated that WWTPs discharge EACs to Minnesota surface waters, information is lacking about the persistence and fate of EACs such as NP, NPEOs, and NPECs in the environment. It is important to determine how far EACs persist downstream from WWTP discharges and if EACs remain in the water column or partition onto bottom sediments. This information is critical to defining the potential risk to aquatic organisms from these chemicals.

Because there is little integrated information about the persistence, fate, and biological responses in field studies,

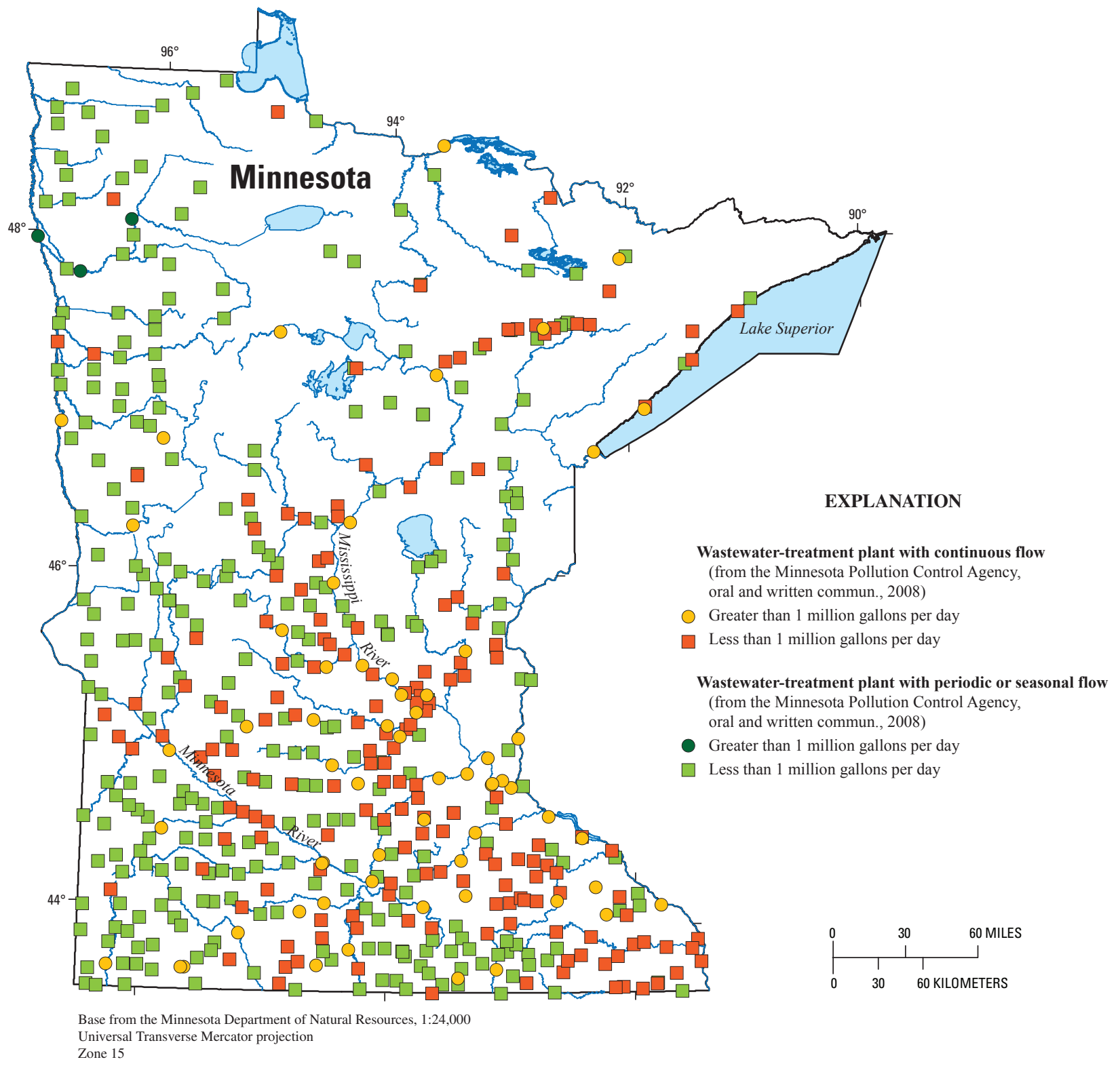

Figure 1. Location of wastewater-treatment plants that discharge to surface waters in Minnesota. 
the U.S. Geological Survey (USGS), in cooperation with the Minnesota Pollution Control Agency and St. Cloud State University, initiated an integrated biological and chemical study of three streams in Minnesota in 2007 to build on previous research from 1994 to 2006 . The objectives of the study were to identify spatial patterns and temporal variability of APs and other EACs and the responses of fish in three small streams receiving wastewater in Minnesota. This study was not designed to assess the efficacy of wastewater treatment practices.

\section{Purpose and Scope}

This report describes the study design and environmental data related to the occurrence of APs and other EACs and fish responses in three small streams receiving wastewater in Minnesota. Data were collected at 13 sites on three streams by the USGS staff and St. Cloud State University staff between February and September 2007. The study design includes site descriptions, sampling methods, and environmental conditions during sampling. Data on hydrologic characteristics, water chemistry, bed-sediment chemistry, and fish characteristics are presented in Microsoft Excel spreadsheets (appendixes 1-10).

\section{Acknowledgments}

The authors would like to thank numerous personnel from the Minnesota Pollution Control Agency, St. Cloud State University, U.S. Geological Survey, Minnesota Department of Natural Resources, and the Marshall, Hinckley, and Hutchinson wastewater-treatment plants, whose contributions made this study possible. Minnesota Pollution Control Agency personnel Angela Preimesberger, Mark Ferrey, David Fawcett, and Carol Sinden contributed to the study design, field sampling, data retrievals, and geographic information system and environmental data analyses. Paul Wingate, Don Pereira, and Jack Enblom at the Minnesota Department of Natural Resources assisted with study design and fish sampling permits and techniques. Personnel at the Marshall, Hinckley, and Hutchinson WWTPs provided site access and details of plant operations. St. Cloud State University personnel Luke A. Vierkant and Brandon P. Johnson assisted with intensive fish collection for the study; Cassandra D. Houdek and Meghan McGee assisted with laboratory analyses; Stephen E. Bartell analyzed fish plasma for Vtg; and Kent Grove, Tim Loes, and Matt Julius provided suggestions throughout the study. USGS personnel Chad Anderson, Christine Yaeger, John Greene, Katie Barnella, Janece Koelis, and Jeff Writer collected and analyzed environmental samples. The authors also thank Richard Kiesling, Janet Carter, and Erik Smith (USGS) for thorough technical reviews of this report, Rebecca Inman for graphic illustration, Mary-Margaret Coates for editorial review, and Felicia Dodd for layout.

\section{Study Design and Approach}

A multicomponent approach was used to accomplish the objectives of this study. The first component was a longitudinal study of three streams receiving WWTP effluent discharge. Stream sampling locations (for water and bed sediment) were established at one location upstream and at two successive locations downstream from WWTP effluent discharge locations on each stream (fig. 2). One exception to this array was that two upstream sampling locations were established on the Grindstone River. In addition, the WWTP effluent discharge pipe was established as a sampling location on each stream. Water samples were collected six times during the study period at all stream and WWTP locations. Bed-sediment samples were collected once during June at all stream locations. This component of the study can be used to characterize differences among streams and associated WWTP effluents, to characterize spatial patterns of selected chemicals in the water column and bed sediment, and to determine temporal variability of the selected chemicals. This component also can allow for investigation of chemicals from possible nonpoint sources through evaluation of the sites upstream from each WWTP.

The second component included a daily sampling (during a time period that coincided with early summer runoff)

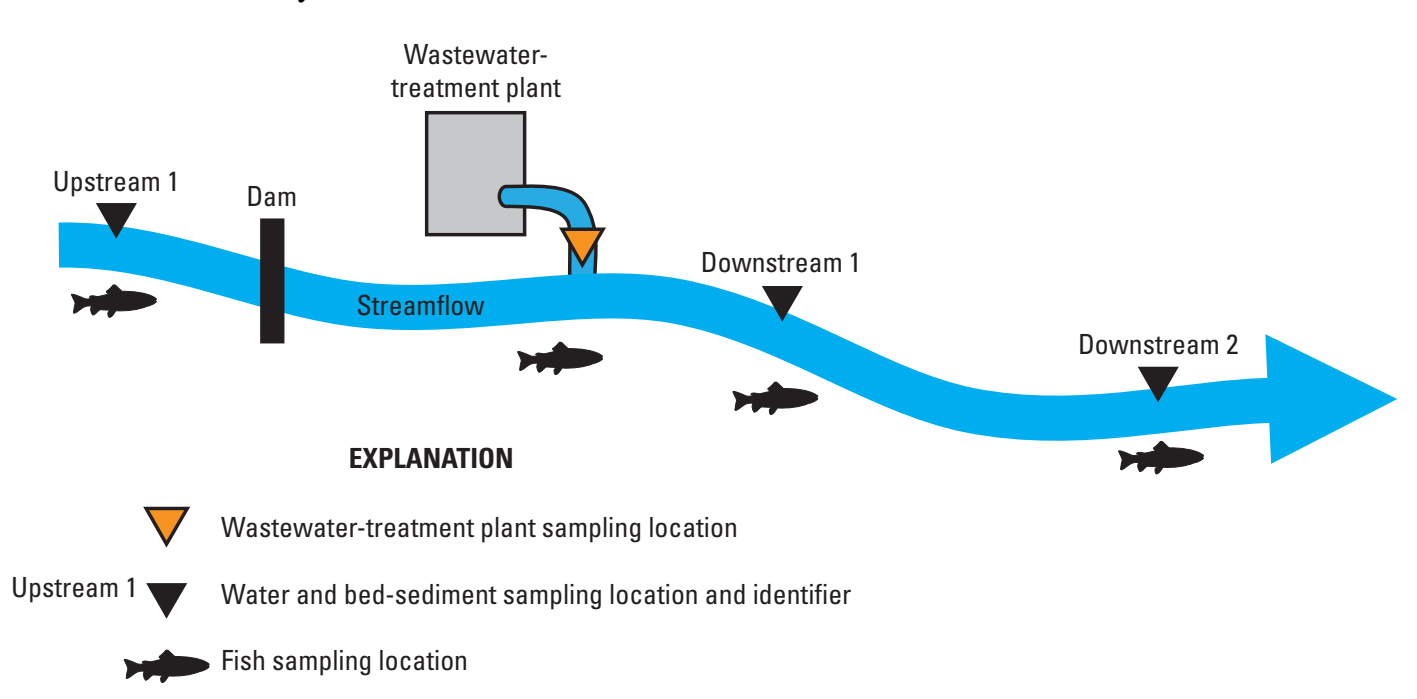

Figure 2. Schematic diagram showing relative locations of sampling sites. 
at the Redwood River near Marshall, Minn., to determine daily fluctuations in total APs and atrazine. The collection of water samples during six periods and the collection of daily samples at one location can improve understanding of the temporal variability in selected chemicals in water and may help to define potential changes in risk to aquatic communities throughout the year.

The third component, accomplished by St. Cloud State University, was collection of multiple fish species at each stream location sampled for water and bed sediment and at one additional location in the stream at the WWTP discharge location during one time period to determine fish responses (Jahns, 2008). Male fish responses were the focus of the third component, although female fish were collected incidentally and for which some biological characterizations were completed.

\section{Site Selection}

Sites were selected through a cooperative effort among USGS, Minnesota Pollution Control Agency, and St. Cloud State University on the basis of results from previous studies in Minnesota (Folmar and others, 1996, 2001; Lee and others, 2004, 2008; Barber and others, 2007). Small streams in Minnesota with homogeneous upstream land cover, at least 5 percent of streamflow contributed by WWTP effluent at the point of discharge, and a dam between the sites upstream and downstream from the WWTP that prevented fish migration were evaluated. Three tributaries in the Mississippi River Basin were selected: South Fork Crow River flowing through Hutchinson, Minn.; the Redwood River flowing through Marshall, Minn.; and the Grindstone River flowing through Hinckley, Minn. (table 1).

Two major environmental settings were targeted: dominant agricultural land use in the upstream basin, and dominant forested and wetland land cover in the upstream basin (table 2). By selecting streams with different upstream land uses, the contribution of selected chemicals from nonpoint sources in various land uses could be investigated.

In addition to the previously mentioned selection criteria, sites with no or few continuously flowing WWTPs upstream from the selected sampling reach and WWTP were desired. This criterion was not possible at all sites; WWTPs are present upstream from the sampling reach on South Fork Crow River and Redwood River.

\section{Environmental Setting}

The South Fork Crow River is a tributary to the Crow River in west-central Minnesota that flows through predomi-

Table 1. Characteristics of sites studied.

[USGS, U.S. Geological Survey; WWTP, wastewater-treatment plant; $\mathrm{mi}^{2}$, square miles; --, not assigned or not determined]

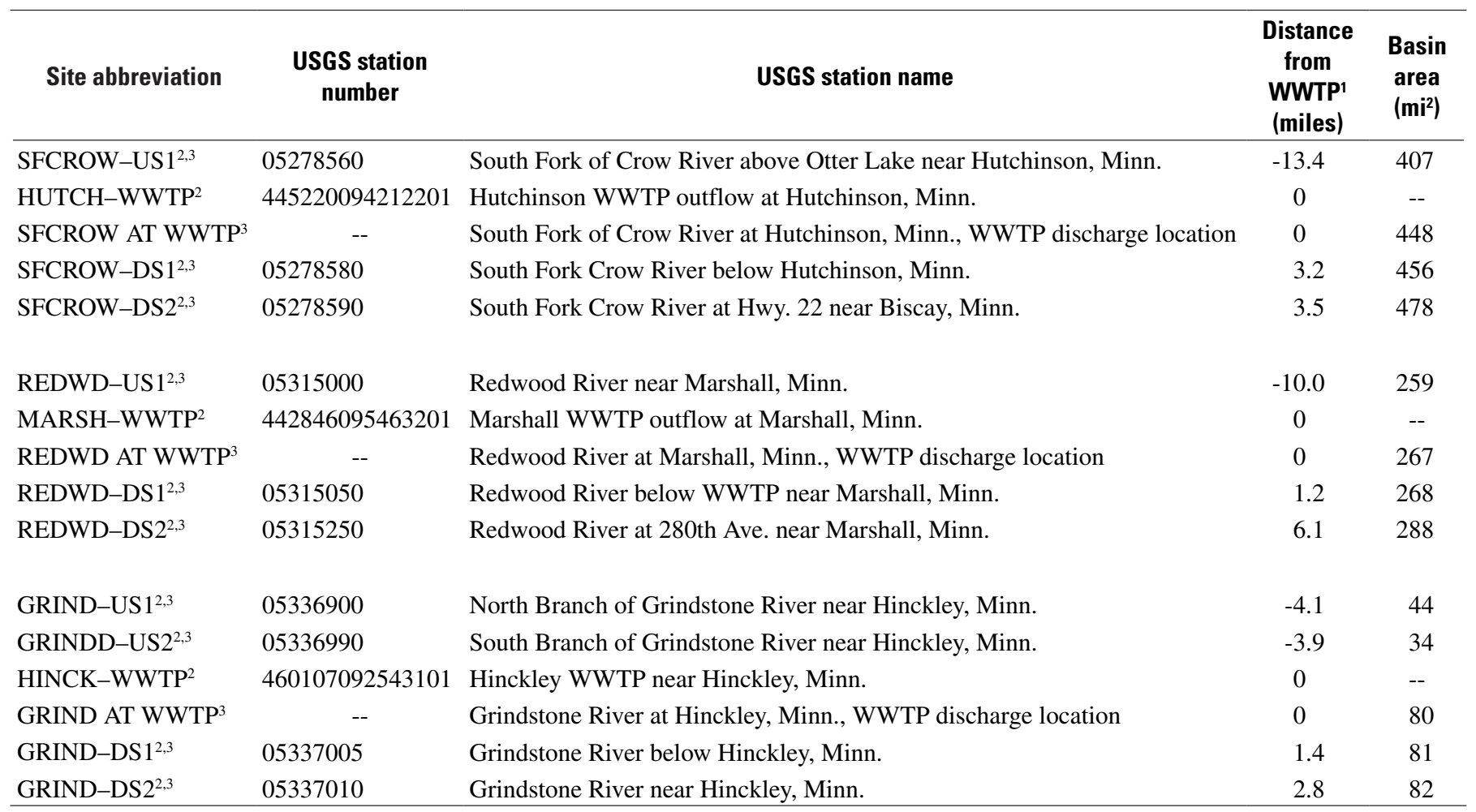

${ }^{1} \mathrm{~A}$ negative value indicates the distance upstream from the wastewater treatment plant discharge.

${ }^{2}$ Water and bed-sediment samples collected at this site.

${ }^{3}$ Fish samples collected at this site. 
Table 2. Land-use characteristics of drainage basins studied.

\begin{tabular}{lrcr}
\hline & \multicolumn{3}{c}{$\begin{array}{c}\text { Drainage basin land use } \\
\text { (percentage of the basin) }\end{array}$} \\
\cline { 2 - 4 } \multicolumn{1}{c}{ Land use $^{1}$} & $\begin{array}{c}\text { South Fork } \\
\text { Crow River }\end{array}$ & $\begin{array}{c}\text { Redwood } \\
\text { River }\end{array}$ & $\begin{array}{c}\text { Grindstone } \\
\text { River }\end{array}$ \\
\hline Urban and rural development & 3.4 & 3.1 & 1.4 \\
Cultivated land & 78.6 & 78.3 & 7.9 \\
Hay, pasture, grassland & 6.6 & 11.8 & 34.2 \\
Brushland & .1 & .1 & 2.7 \\
Forested & 3.5 & 3.0 & 38.4 \\
Water & 5.4 & 2.9 & 2.9 \\
Bog/marsh/fen & 2.3 & .7 & 12.3 \\
Mining/extraction & .1 & .1 & .2 \\
\hline
\end{tabular}

${ }^{1}$ Land use for the drainage basin upstream from the most downstream site on each stream was determined with geographic information system analyses from the Minnesota Department of Natural Resources Land Use and Cover Census of the 1990s raster data.

nantly agricultural land planted in corn, soybeans, alfalfa, spring wheat, and oats (Minnesota Pollution Control Agency, 2000; U.S. Department of Agriculture, 2006; fig. 3, table 2). This stream originates upstream of small headwater lakes. The upstream site on the South Fork Crow River is upstream from Lake Otter, which is formed behind a low-head dam in Hutchinson, Minn. The Hutchinson WWTP effluent discharge enters the South Fork Crow River downstream from the dam, and the two downstream sampling sites are downstream from the city of Hutchinson. Effluents from small WWTPs are discharged upstream from the sampling reach. The most upstream WWTP is the city of Kandiyohi WWTP; it has a continuousdischarge design flow of $0.112 \mathrm{Mgal} / \mathrm{d}$ (Minnesota Pollution Control Agency, written commun., 2008). The city of Lake Lillian has the next downstream WWTP discharge followed by discharges from Cosmos and Cedar Mills, both of which use controlled releases (treated wastewater is released periodically) of less than $0.09 \mathrm{Mgal} / \mathrm{d}$ (Minnesota Pollution Control Agency, written commun., 2008).

The South Fork Crow River Basin is developed on glacial deposits of the Des Moines Lobe of the Wisconsinage ice sheet; it consists primarily of calcareous deposits containing limestone and shale (Minnesota Pollution Control Agency, 2000). The basin is characterized by a nearly level to gently sloping, dissected glaciated plain (U.S. Department of Agriculture, 2006). Soils in the basin are primarily mollisols that typically form on prairies underlain by calcareous sediments (Minnesota Pollution Control Agency, 2000). The average annual precipitation is 27 to 29 inches (in.), and most of the precipitation falls during the growing season (Minnesota Pollution Control Agency, 2000). In most of the region, the average annual temperature is 46 to 54 degrees Fahrenheit $\left({ }^{\circ} \mathrm{F}\right)$.
The Hutchinson WWTP is a Class A facility with a continuous discharge to the South Fork Crow River (Minnesota Pollution Control Agency, 2007). A main lift station with four pumps and two mechanically cleaned bar screens provides raw wastewater to the WWTP. The WWTP is designed to treat and discharge an average wet-weather flow of $4.27 \mathrm{Mgal} / \mathrm{d}$ (table 3). The facility is designed to treat a 5-day carbonaceous biochemical oxygen demand (CBOD5) influent strength of 223 milligrams per liter (mg/L) (Minnesota Pollution Control Agency, 2007). The facility consists of a flow equalization basin, four static fine screens, three grit removal units, two oxidation ditches, two secondary clarifiers, a chlorination and dechlorination disinfection system, and two re-aeration units.

Similar to the South Fork Crow River, the Redwood River also drains predominantly agricultural land (table 2, fig. 4). Narrow wooded strips generally line streams and rivers or farmstead boundaries (U.S. Department of Agriculture, 2006). The area was originally covered by native prairie vegetation and wet prairies (Patterson, 1997). The Redwood River originates in the Prairie Coteau, which is a highland resulting from the last continental glaciation (U.S. Department of Agriculture, 2006). The Redwood River drops nearly 300 feet in 15 miles; however, as the river approaches Marshall, Minn., the gradient is reduced to 2-3 feet per mile (Minnesota Pollution Control Agency, 2008). Glacial drift covers most of the drainage basin (Minnesota Pollution Control Agency, 2008) and consists of mostly clay and till with local deposits of sand and gravel with a clay-loam texture (Patterson, 1997).

Average annual precipitation in the area ranges from 19 to 29 in. (U.S. Department of Agriculture, 2006). Similar to precipitation in the South Fork Crow River drainage basin, one-half or more of the precipitation in the Redwood River drainage basin falls during the growing season as highintensity thunderstorms during the summer; precipitation in winter is mostly snow (U.S. Department of Agriculture, 2006). The range in average annual temperature is 38 to $45^{\circ} \mathrm{F}$ (U.S. Department of Agriculture, 2006). Several small WWTPs with controlled discharges are upstream from the sampling reach: Ruthton WWTP (which has a wet-weather design flow of $0.05 \mathrm{Mgal} / \mathrm{d})$, Tyler WWTP (0.175 Mgal/d), Russell WWTP (0.084 Mgal/d), and Lynd WWTP (0.045 Mgal/d) (Minnesota Pollution Control Agency, written commun., 2008). These WWTPs released water from ponds during April and May of the 2007 study period.

The Marshall WWTP is a Class A facility that consists of two main lift stations, a grinder, a cyclone grit chamber, two primary clarifiers, two trickling filter towers, an intermediate clarifier, four extended aeration basins, two final clarifiers, a solids contact clarifier, two dual-media filters, ultraviolet (UV) disinfection facilities, an effluent aeration channel, two primary anaerobic digesters, one secondary anaerobic digester, three sludge storage tanks, sludge thickening facilities, and two equalization basins (Minnesota Pollution Control Agency, 2004). The treatment system has a continuous discharge to the Redwood River and is designed to treat an average wet- 

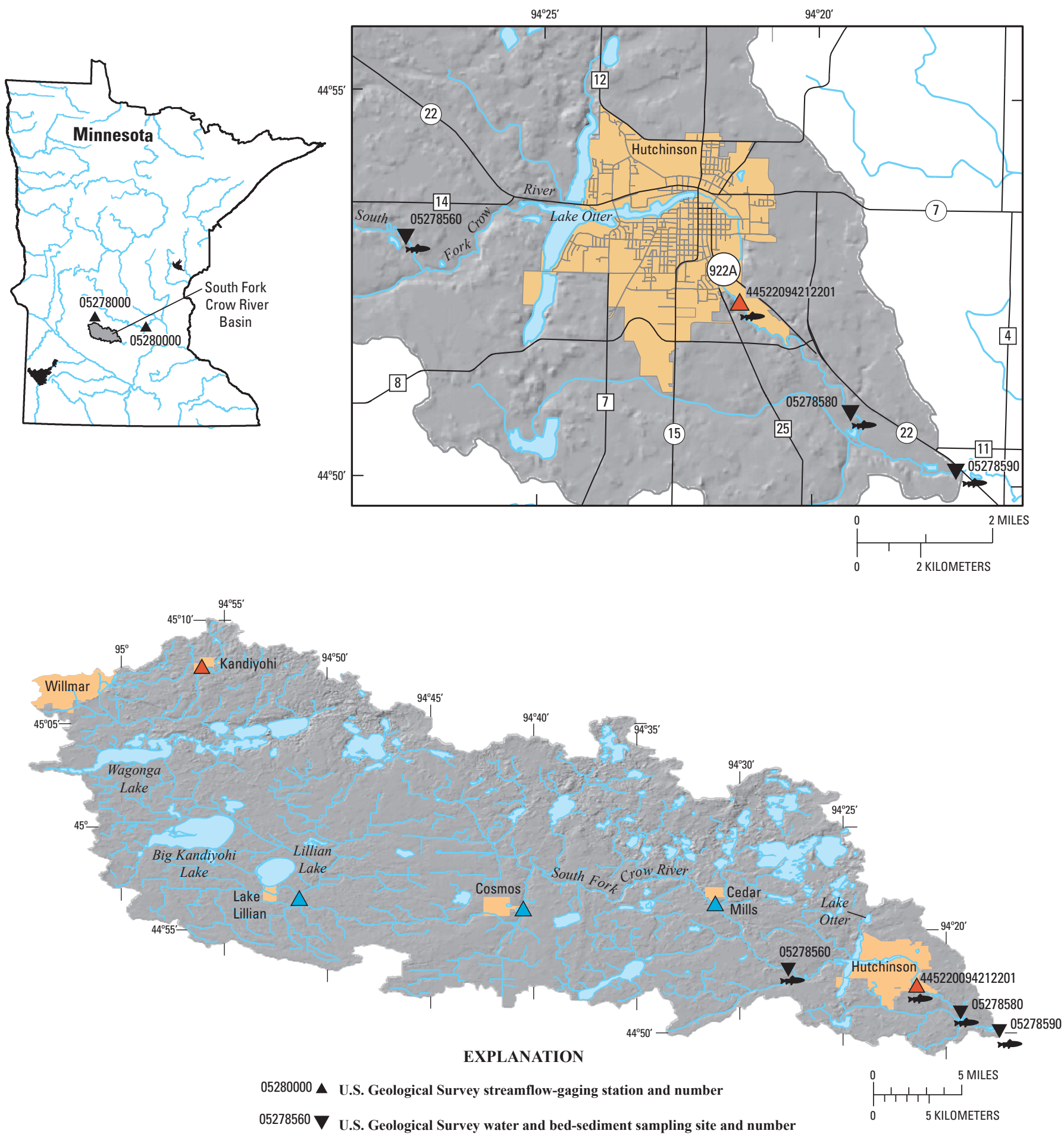

$44522094212201 \triangle$ Sampling site and number for wastewater-treatment plant with continuous flow (site without number was not sampled)

$\triangle$ Wastewater-treatment plant with periodic or seasonal flow (site not sampled)

St. Cloud State University fish sampling site

Figure 3. South Fork Crow River drainage basin, wastewater-treatment plants, and sampling sites along the sampling reach. 
Table 3. Characteristics of wastewater-treatment plants studied. Data are from Minnesota Pollution Control Agency, written commun., 2008.

[Mgal/d, million gallons per day; WWTP, wastewater-treatment plant; NPDES, National Pollutant and Discharge Elimination System]

\begin{tabular}{|c|c|c|c|c|c|c|c|}
\hline \multirow{2}{*}{$\begin{array}{l}\text { Wastewater- } \\
\text { treatment } \\
\text { plant }\end{array}$} & \multirow{2}{*}{$\begin{array}{c}\text { Population } \\
\text { served }\end{array}$} & \multicolumn{2}{|c|}{ Average WWTP design flow } & \multicolumn{2}{|c|}{ Inflow } & \multirow{2}{*}{$\begin{array}{c}\text { NPDES } \\
\text { identification } \\
\text { number }\end{array}$} & \multirow{2}{*}{$\begin{array}{c}\text { Major } \\
\text { industrial } \\
\text { inputs }\end{array}$} \\
\hline & & $\begin{array}{c}\text { Wet } \\
\text { (Mgal/d) }\end{array}$ & $\begin{array}{c}\text { Dry } \\
\text { (Mgal/d) }\end{array}$ & $\begin{array}{l}\text { Domestic } \\
\text { (percent) }\end{array}$ & $\begin{array}{l}\text { Industrial } \\
\text { (percent) }\end{array}$ & & \\
\hline Hutchinson & 13,977 & 4.27 & 2.22 & 68 & 32 & MN-0055832 & $\begin{array}{l}\text { Electrical and electronic compo- } \\
\text { nents, metal finishing, electro- } \\
\text { plating, and food processing. }\end{array}$ \\
\hline Marshall & 13,031 & 4.5 & 3.31 & 30 & 70 & MN-0022179 & Food processing, corn processing \\
\hline Hinckley & 1,438 & .68 & .57 & 60 & 40 & MN-0023701 & Casino, restaurants. \\
\hline
\end{tabular}

weather flow of as much as $4.5 \mathrm{Mgal} / \mathrm{d}$ (table 3) with CBOD5 strength of $319 \mathrm{mg} / \mathrm{L}$ (Minnesota Pollution Control Agency, 2004). The average dry-weather design flow is $3.31 \mathrm{Mgal} / \mathrm{d}$. An industrial wastewater discharge from a wet corn milling operation is located directly upstream from the WWTP discharge and had a daily average flow of $1.5 \mathrm{Mgal} / \mathrm{d}$ during this study (Minnesota Pollution Control Agency, 2004).

The Grindstone River is located in east-central Minnesota and is a tributary to the Kettle River, which is a tributary to the St. Croix River (fig. 5). The Grindstone River flows through predominantly forested (deciduous and coniferous) land with a substantial percentage of wetlands and some agriculture in the downstream portions of the basin (table 2). The drainage basin was previously covered by virgin white pine, which was removed through logging by 1887 , and currently is covered by the second-growth vegetation of maple basswood, aspen, birch, and oak (Waters, 1977; U.S. Department of Agriculture, 2006).

The two main tributaries to the Grindstone River are the North and South Branches of the Grindstone River. The North Branch flows from Grindstone Lake and has more forested and wetland land use than does the South Branch. The two branches converge in Hinckley and flow into a small lake within Hinckley that has a low-head dam. The main channel of the Grindstone River starts downstream from that dam.

During Wisconsian glaciation, bedrock in the area was covered with sediments deposited by the Superior Lobe; the deposits are primarily siliceous (U.S. Department of Agriculture, 2006). The glacial deposits formed an almost continuous blanket as much as several hundred feet thick in many areas. The area is characterized by outwash plains, dunes, lake plains, swamps, bogs, and marshes. Lakes are common, and streams generally form a dendritic pattern. The average annual precipitation in this area is 26 to $34 \mathrm{in}$. (U.S. Department of Agriculture, 2006). Precipitation commonly occurs as convective thunderstorms during the growing season; snow generally falls from October through April. The average annual temperature is 38 to $45^{\circ} \mathrm{F}$ (U.S. Department of Agriculture, 2006). No WWTPs are upstream from the sampling locations.

The Hinckley WWTP is a Class B facility treatment system consisting of a mechanical fine screen with a backup manual bar screen, two extended aeration-activated sludge ponds totaling 700,000 gallons, based on a 24 -hour hydraulic detention time, one 45-foot-diameter final clarifier, one 28,000-cubic-foot aerated digester basin, a biosolids storage lagoon, chemical phosphorus removal, and UV disinfection (Minnesota Pollution Control Agency, 2005). One of the old aeration ponds functions as an equalization basin for the UV disinfection system. During periods of heavy precipitation, water can be retained in the pond to avoid exceeding the capacity of the UV system. The aerated pond also may be used as a clarifier if the final clarifier needs to be temporarily removed from service. The facility has a continuous discharge to the Grindstone River with an average wet-weather design flow of $0.68 \mathrm{Mgal} / \mathrm{d}$, an average dry weather design flow of $0.57 \mathrm{Mgal} / \mathrm{d}$, and an influent design CBOD5 strength of 229 mg/L (Minnesota Pollution Control Agency, 2005).

\section{Collection and Analysis Methods}

This section describes the methods used to collect and analyze water and bed-sediment samples. Collection methods for the characterization of fish also are described.

\section{Streamflow Measurement and Calculation}

Streamflow was measured at each site during each sampling event following USGS protocols (Rantz and others, 1982a, b; Morlock and others, 2002) (fig. 6). WWTP daily discharge values were obtained from each WWTP or from the Minnesota Pollution Control Agency. Streamflow at the WWTP effluent discharge location was calculated. The calculations for the streamflow at the WWTP effluent discharge location for the South Fork Crow and Redwood Rivers were determined through linear interpolation between the upstream 

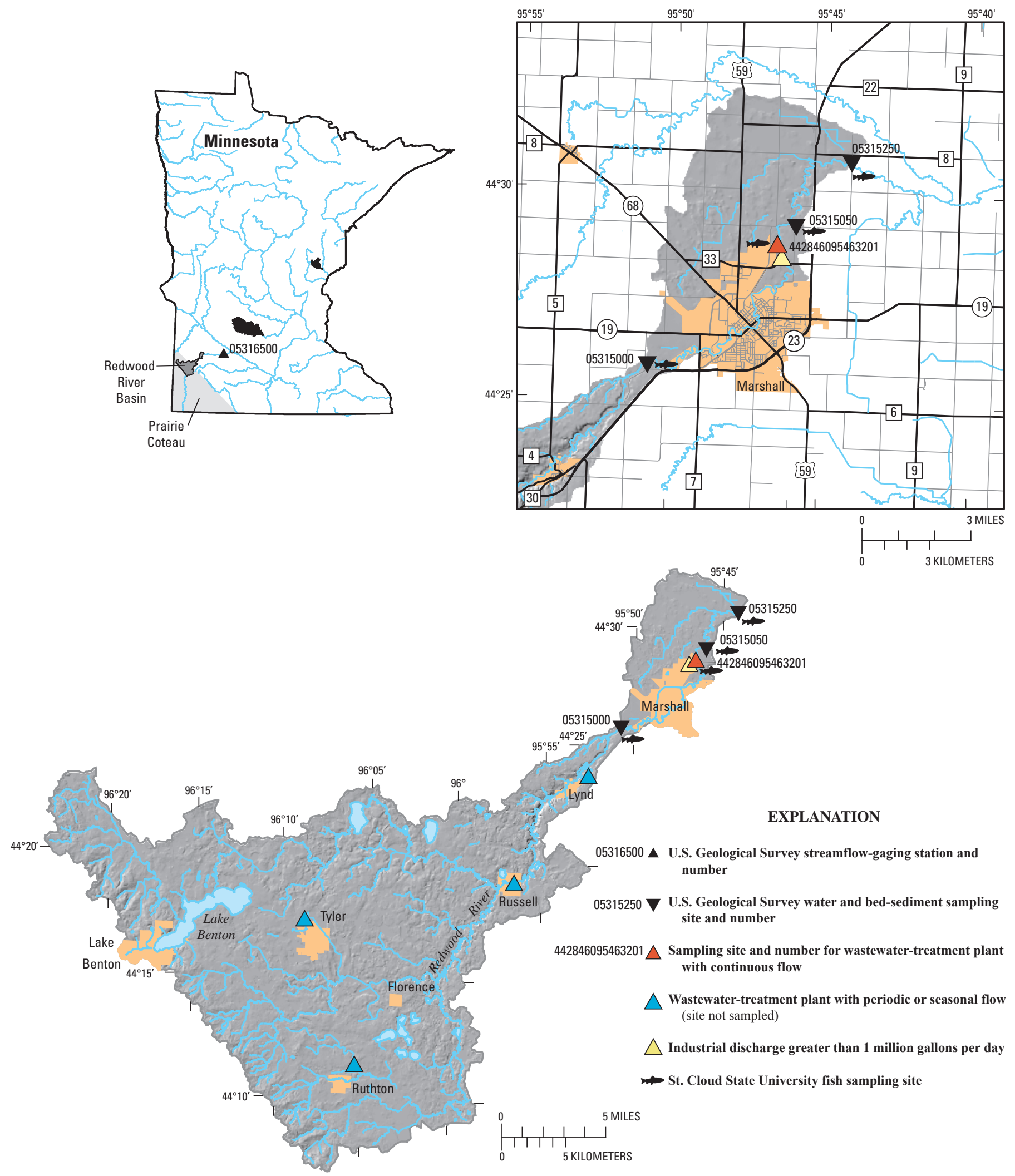

Figure 4. Redwood River drainage basin, wastewater-treatment plants, and sampling sites along the sampling reach. 


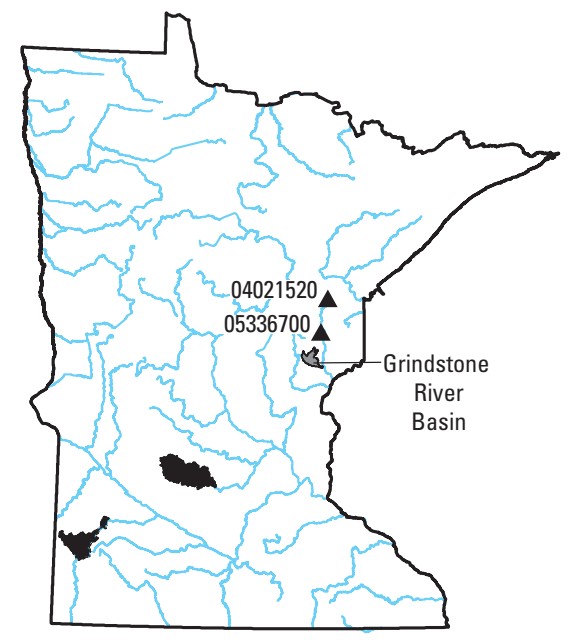

Minnesota
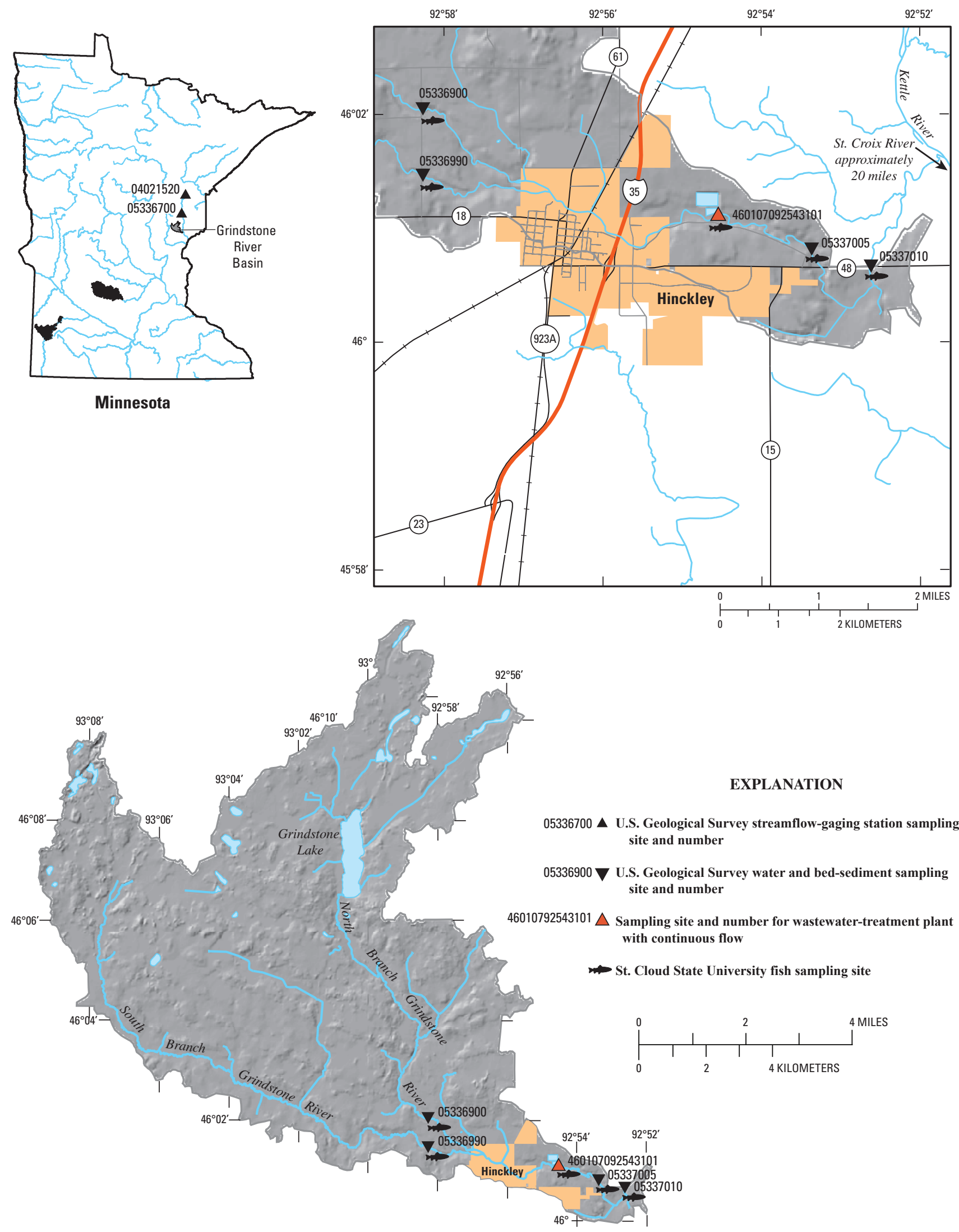

Figure 5. Grindstone River drainage basin, wastewater-treatment plants, and sampling sites along the sampling reach. 

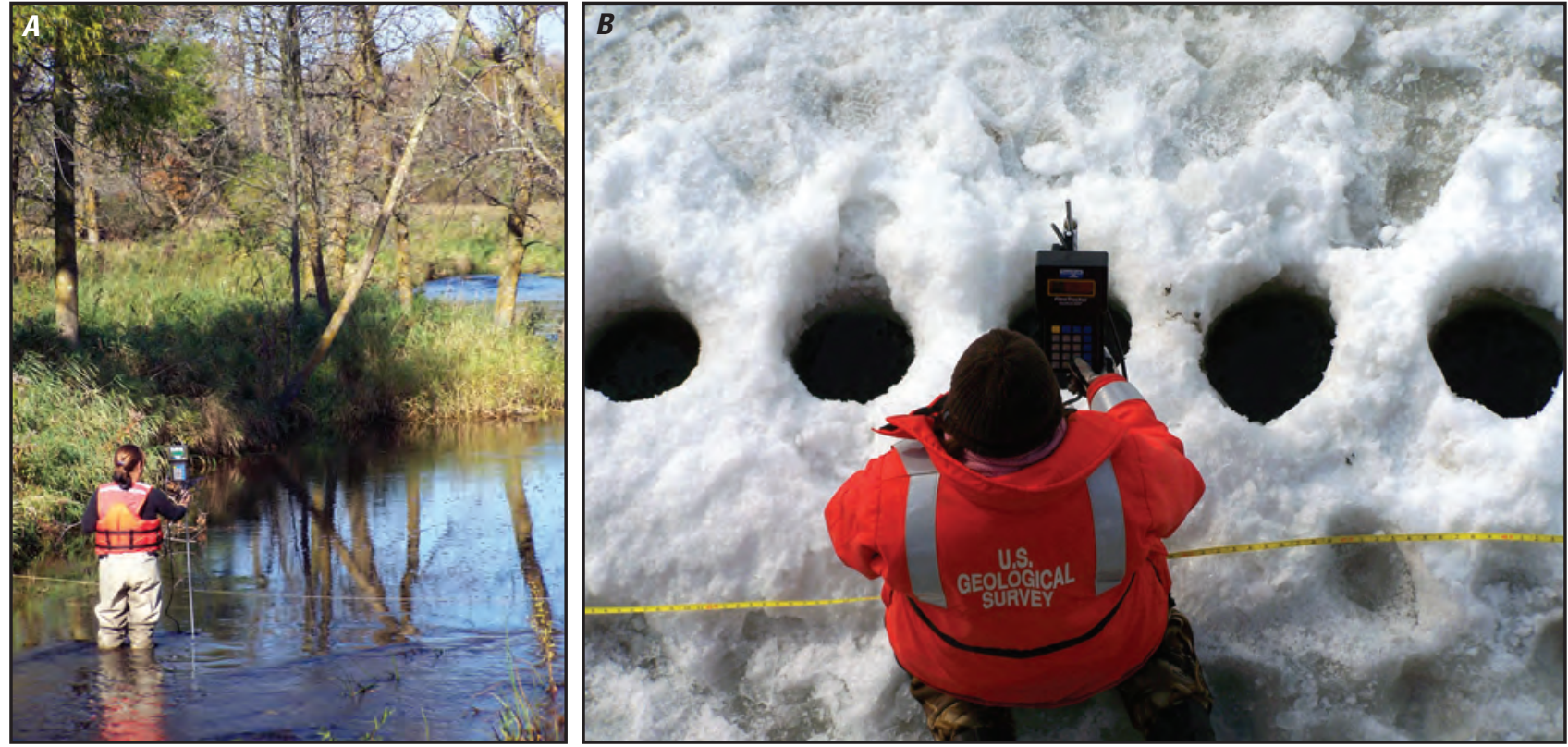

Figure 6. Streamflow measurements at $A$, the Grindstone River, September 2007, and $B$, the South Fork Crow River under ice, February 2007.

and the first downstream sampling location by using the following equation:

$$
Q_{\text {WWTP }}=Q_{\text {upstream }}+\left[\frac{\left(D A_{\text {WWTP }}-D A_{\text {upstream }}\right)}{\left(D A_{\text {downstream }}-D A_{\text {upstream }}\right)} \times\left(Q_{\text {dowsstream }}-Q_{\text {upstream }}\right)\right]
$$

where $Q$ is streamflow in cubic feet per second, and $D A$ is the drainage area in square miles. The discharge from the WWTP was subtracted from the discharge at the downstream sampling location for these calculations. The Grindstone River streamflow at the WWTP discharge location was determined by subtracting the WWTP discharge from the discharge at the first site downstream from the WWTP; the difference in drainage basin area between those two locations was less than 1 square mile, and no tributaries intervene.

\section{Water Sample Collection}

The USGS collected water samples for routine sampling six times from February through September 2007 to capture a variety of seasons and streamflow conditions. Samples were collected at the upstream site (or at the two upstream sites for the Grindstone River), at the wastewater treatment effluent discharge pipe, and at two downstream sites during each sampling period. The stream was first sampled before snowmelt; samples were collected under ice at all sites (fig. 7). The stream was sampled a second time during the spring snowmelt at most sites, and the remaining sampling events occurred during summer and autumn. Water was sampled more frequently at the Redwood River near Marshall, Minn., during spring and summer runoff to determine temporal variability in the occurrence and concentrations of total alkylphenols and atrazine.
Water samples were acquired by use of an automatic sampler (fig. 8), which collected daily samples for a period of 8 weeks (April through July 2007).

To ensure that the samples collected were representative of water flowing in the entire stream cross section, water samples were collected using integrated width-and-depth sampling techniques (Edwards and Glysson, 1988; U.S. Geological Survey, 2003). WWTP samples were collected directly from effluent outflows. Physical measurements of water quality (dissolved oxygen, $\mathrm{pH}$, specific conductance, and water temperature) were made using a submersible YSI data sonde. The sonde was calibrated according to U.S. Geological Survey

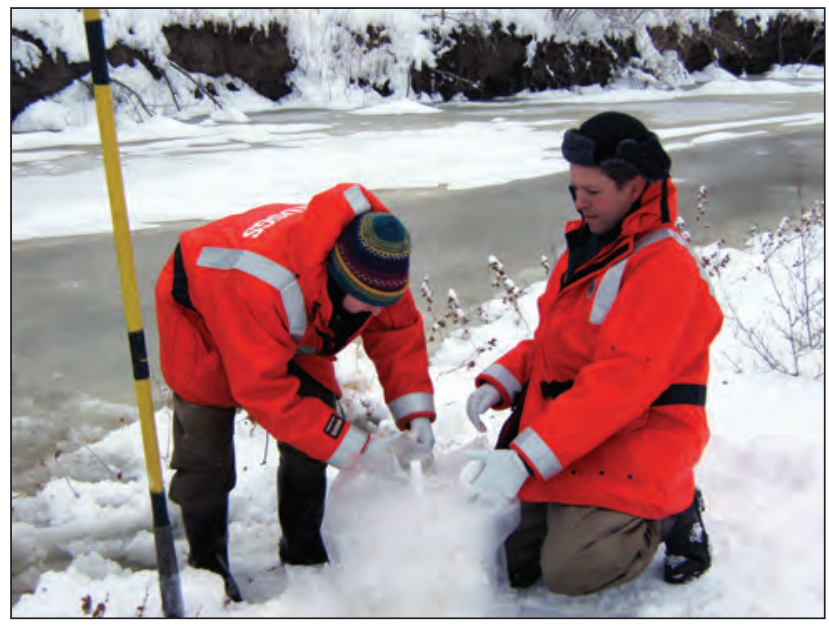

Figure 7. Water collection at the Redwood River near Marshall, Minnesota, February 2007. 


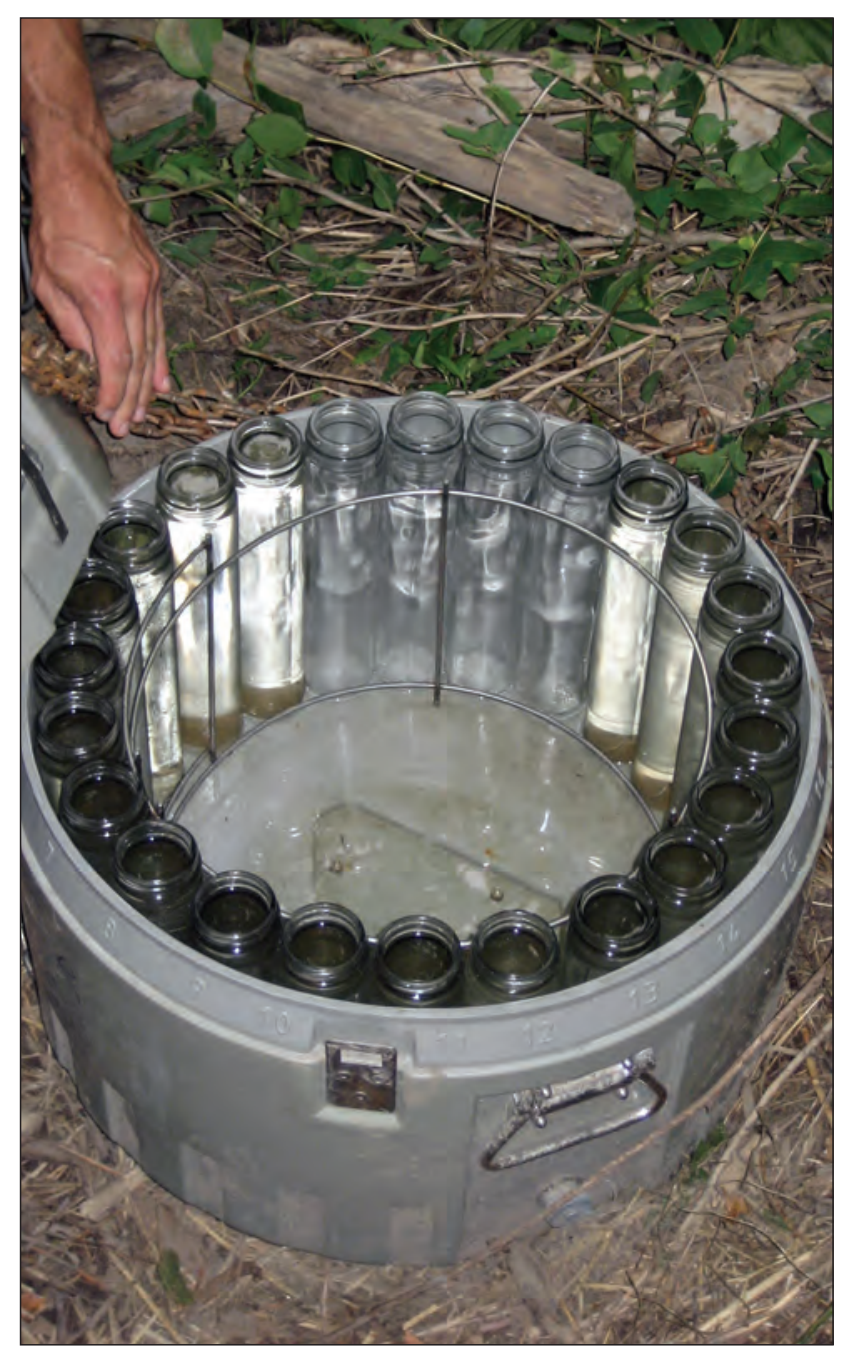

Figure 8. Collection bottles inside the auto sampler used to collect samples at the Redwood River near Marshall, Minnesota, 2007.

(2003) and manufacturer's specifications before and after sampling to ensure accurate measurements.

To avoid contamination of samples, personnel who collected and processed samples avoided use of personal-care items such as insect repellent, sunscreen, cologne, aftershave, and perfume; they did not consume caffeinated products or tobacco products during (or immediately before) collection or processing of samples; and they wore powderless, disposable gloves during sample collection. All samples were collected with inert materials such as Teflon, glass, or stainless steel. All collection and processing equipment was cleaned between samples with a succession of native water, soapy tap water, tap water, deionized water, methanol, and organic-free water rinses. Following collection, chilled samples were processed within 1 to 2 hours after collection before they were shipped to the laboratory.

\section{Water Sample Analyses}

A wide variety of chemicals including major ions, nutrients, organic carbon, and wastewater-derived chemicals (such as fragrances, pesticides, metal complexing agents, surfactant degradation products, plastic components, fire retardants, antioxidants, caffeine, antimicrobials, and steroids) were evaluated in this study (table 4). Major ions occur naturally in water as a result of geochemical weathering of rocks, surface runoff, and atmospheric deposition, and as a result of human activities (Hem, 1985). Water from different geologic settings commonly will have distinctly different major-ion signatures that can be used to infer the source of water (Hem, 1985). Nutrients are compounds that contain nitrogen $(\mathrm{N})$ or phosphorus (P). Natural organic matter in streams comes from internal sources such as primary productivity and from external sources including runoff that carries soils and plants into surface water and point-source inputs such as WWTP discharge to streams. Organic matter is important in streams because it can control the fate of contaminants, light penetration, and $\mathrm{pH}$ (Thurman, 1985; Weishaar and others, 2003). Total organic carbon (TOC) is the sum of dissolved organic carbon and particulate organic carbon. Dissolved organic carbon (DOC) is the organic carbon that passes through a 0.7 micrometer $(\mu \mathrm{m})$ pore-size glass fiber filter. DOC composes most of the organic matter in stream samples, and concentrations range from $0.5 \mathrm{mg} / \mathrm{L}$ in ground water to more than $30 \mathrm{mg} / \mathrm{L}$ in wetlands, whereas DOC in rivers typically ranges from 2 to $10 \mathrm{mg} / \mathrm{L}$ (Thurman, 1985). Specific organic contaminants compose less than 5 percent of the DOC of most waters and typically less than 1 percent (Thurman, 1985; Barber and others, 2001).

A broad suite of organic chemicals that are indicators of industrial, domestic, and agricultural wastewaters (wastewater indicator chemicals) were selected for this study on the basis of usage, toxicity, potential estrogenic activity, and persistence in the environment (Barnes and others, 2002; Kolpin and others, 2002). For example, ethylenediaminetetraacetic acid (EDTA) was included in the analyses because it is a high-production-volume chemical used to form water-soluble complexes with trace metals (Barber, Keefe, and others, 2003). EDTA occurs at relatively high concentrations and can persist in the aquatic environment (Barber and others, 1996, 2000; Leenheer and others, 2001; Barber, Keefe, and others, 2003). Additional chemicals such as plastic components, fire retardants, caffeine, triclosan, and synthetic musk compounds are included because they are commonly detected in or downstream from WWTPs, which makes them indicators of municipal wastewater effluent (Glassmeyer and others, 2005).

USGS research and production methods were used to analyze the chemicals in water and bed sediment for this study. Two USGS laboratories analyzed water and bedsediment samples for this study. The USGS National Water Quality Laboratory (NWQL) in Denver, Colo., analyzed water samples for major ions, nutrients, and organic wastewater indicators by using official production methods. The USGS National Research Program Laboratory (USGS-NRP) in 
Table 4. Physical properties and chemicals analyzed for in water samples.

[MRL, minimum reporting level; EDP, endocrine-disrupting potential; $K_{o w}$, octanol-water partition coefficient; CASRN, Chemical Abstracts Services Registry Number; $\mu$ S/cm, microsiemens per centimeter at 25 degrees Celsius ( $\left.{ }^{\circ} \mathrm{C}\right)$; FIELD, field sample; mg/L, milligrams per liter; --, not available; NWQL, National Water Quality Laboratory; USGS-NRP, U.S. Geological Survey National Research Program (laboratory); EDTA, ethylenediaminetetraacetic acid; NTA; nitrilotriacetic acid; NPEC, nonlyphenol ethoxycarboxylate; $\mu \mathrm{g} / \mathrm{L}$, micrograms per liter; ng/L, nanograms per liter; S, suspected; K, known; E or e, remark code estimated concentration reported; F, fungicide; H, herbicide; I, insecticide; GUP, general-use pesticide; FR, flame retardant; WW, wastewater; manuf., manufacturing; \%, percent; >, greater than; CP, combustion product; PAH, polycyclic aromatic hydrocarbon; UV, ultraviolet; NA, not applicable]

\begin{tabular}{|c|c|c|c|c|c|c|}
\hline Property/chemical name & Abbreviation & MRL'1 & EDP2 $^{2}$ & $\log K_{\mathrm{ow}}{ }^{3}$ & CASRN $^{4}$ & Possible compound uses or sources ${ }^{5}$ \\
\hline \multicolumn{7}{|c|}{ Field measurements } \\
\hline Specific conductance $(\mu \mathrm{S} / \mathrm{cm})$ & $\mathrm{SC}$ & -- & -- & -- & -- & Field measurement. \\
\hline Dissolved oxygen $(\mathrm{mg} / \mathrm{L})$ & DO & -- & -- & -- & -- & Field measurement. \\
\hline $\mathrm{pH}$ & $\mathrm{pH}$ & -- & -- & -- & -- & Field measurement. \\
\hline Water temperature $\left({ }^{\circ} \mathrm{C}\right)$ & TEMP & -- & -- & -- & -- & Field measurement. \\
\hline \multicolumn{7}{|c|}{ USGS-NWQL major ions and nutrients (mg/L) } \\
\hline Calcium & $\mathrm{CA}$ & 0.04 & -- & -- & $7440-70-2$ & Mineral. \\
\hline Magnesium & MG & .02 & -- & -- & $7439-95-4$ & Mineral. \\
\hline Potassium & K & .02 & -- & -- & $7440-09-7$ & Mineral. \\
\hline Sodium & NA & .12 & -- & -- & $7440-23-5$ & Mineral. \\
\hline Chloride & CL & .12 & -- & -- & $16887-00-6$ & Mineral. \\
\hline Fluoride & FL & .12 & -- & -- & $16984-48-8$ & Mineral. \\
\hline Silica & SI & .018 & -- & -- & $7631-86-9$ & Mineral. \\
\hline Sulfate & SU & .18 & -- & -- & $14808-79-8$ & Mineral. \\
\hline Iron & FE & 8 & -- & -- & $7439-89-6$ & Mineral. \\
\hline Manganese & MN & .4 & -- & -- & $7439-96-5$ & Mineral. \\
\hline Ammonia as nitrogen & $\mathrm{NH}_{3}$ & .02 & -- & -- & $7664-41-7$ & Nutrient. \\
\hline Nitrite plus nitrate & $\mathrm{NO}_{2}+\mathrm{NO}_{3}$ & .004 & -- & -- & -- & Nutrient. \\
\hline Nitrogen, nitrite & $\mathrm{NO}_{2}$ & .002 & -- & -- & $14797-65-0$ & Nutrient. \\
\hline Total nitrogen $\left(\mathrm{NH}_{3}+\mathrm{NO}_{2}+\mathrm{NO}_{3}+\right.$ organic $)$ & $\mathrm{TN}$ & .06 & -- & -- & $17778-88-0$ & Nutrient. \\
\hline Orthophosphate & ORTHOP & .006 & -- & -- & $14265-44-2$ & Nutrient. \\
\hline Total phosphorus & $\mathrm{TP}$ & & & -- & 7723-14-0 & Nutrient. \\
\hline \multicolumn{7}{|c|}{ USGS-NRP organic carbon } \\
\hline Total organic carbon $(\mathrm{mg} / \mathrm{L})$ & TOC & 0.5 & -- & -- & -- & Natural organic matter. \\
\hline Ultraviolet absorbance at $254 \mathrm{~nm}$ & $\mathrm{UV}_{254}$ & .001 & -- & -- & -- & Natural organic matter. \\
\hline Specific ultraviolet absorbance at $254 \mathrm{~nm}$ & SUVA $_{254}$ & -- & -- & -- & -- & Natural organic matter. \\
\hline \multicolumn{7}{|c|}{ USGS-NRP EDTA, NTA, NPEC ( $\mu \mathrm{g} / \mathrm{L})$} \\
\hline Ethylenediaminetetraacetic acid & EDTA & 0.5 & -- & -3.86 & $60-00-4$ & Metal complexing agent. \\
\hline Nitrilotriacetic acid & NTA & .5 & -- & -3.81 & $139-13-9$ & Metal complexing agent. \\
\hline 4-Nonylphenolmonoethoxycarboxylate & NP1EC & 5 & -- & 5.80 & $3115-49-9$ & Surfactant metabolite. \\
\hline 4-Nonylphenoldiethoxycarboxylate & NP2EC & 5 & -- & 5.53 & $106807-78-7$ & Surfactant metabolite. \\
\hline 4-Nonylphenoltriethoxycarboxylate & NP3EC & 5 & -- & -- & $108149-59-3$ & Surfactant metabolite. \\
\hline 4-Nonylphenoltetraethoxycarboxylate & NP4EC & 5 & -- & -- & -- & Surfactant metabolite. \\
\hline 4- $n$-Nonylphenolmonoethoxycarboxylate & nNP1EC & -- & -- & -- & -- & Surrogate standard. \\
\hline
\end{tabular}


Table 4. Physical properties and chemicals analyzed for in water samples.-Continued

[MRL, minimum reporting level; EDP, endocrine-disrupting potential; $K$, octanol-water partition coefficient; CASRN, Chemical Abstracts Services Registry Number; $\mu$ S/cm, microsiemens per centimeter at 25 degrees Celsius ( $\left.{ }^{\circ} \mathrm{C}\right)$; FIELD, field sample; mg/L, milligrams per liter; --, not available; NWQL, National Water Quality Laboratory; USGS-NRP, U.S. Geological Survey National Research Program (laboratory); EDTA, ethylenediaminetetraacetic acid; NTA; nitrilotriacetic acid; NPEC, nonlyphenol ethoxycarboxylate; $\mu \mathrm{g} / \mathrm{L}$, micrograms per liter; ng/L, nanograms per liter; S, suspected; K, known; E or e, remark code estimated concentration reported; F, fungicide; H, herbicide; I, insecticide; GUP, general-use pesticide; FR, flame retardant; WW, wastewater; manuf., manufacturing; \%, percent; >, greater than; CP, combustion product; PAH, polycyclic aromatic hydrocarbon; UV, ultraviolet; NA, not applicable]

\begin{tabular}{|c|c|c|c|c|c|c|}
\hline Property/chemical name & Abbreviation & MRL'1 $^{1}$ & EDP $^{2}$ & $\log K_{o w}{ }^{3}$ & CASRN $^{4}$ & Possible compound uses or sources ${ }^{5}$ \\
\hline \multicolumn{7}{|c|}{ USGS-NRP wastewater indicators (ng/L) } \\
\hline 1,2-Dichlorobenzene & $1,2 \mathrm{DCB}$ & 5 & -- & 3.28 & $95-50-1$ & Fumigant. \\
\hline 1,3-Dichlorobenzene & $1,3 \mathrm{DCB}$ & 5 & -- & 3.28 & $541-73-1$ & Fumigant. \\
\hline 1,4-Dichlorobenzene ${ }^{6}$ & $1,4 \mathrm{DCB}$ & 5 & S & 3.28 & $106-46-7$ & Moth repellent, fumigant, deodorant. \\
\hline 2,6-Di-tert-butyl-1,4-benzoquinone & DTBB & 5 & -- & 4.07 & $719-22-2$ & Antioxidant by-product. \\
\hline 2,6-Di-tert-butyl-4-methylphenol & BHT & 5 & -- & 5.03 & $128-37-0$ & Antioxidant. \\
\hline 2,6-Di-tert-butylphenol & DTBP & 5 & -- & 4.48 & $128-39-2$ & Antioxidant. \\
\hline 3-beta-Coprostanol ${ }^{6}$ & COP & 5 & -- & 8.82 & $360-68-9$ & Animal fecal steroid. \\
\hline 3-tert-Butyl-4-hydroxyanisole ${ }^{6}$ & BHA & 5 & S & 3.50 & $25013-16-5$ & Antioxidant. \\
\hline 4-Ethylphenol & EP & 5 & -- & 2.55 & $123-07-9$ & Plasticizer \\
\hline 4-Methylphenol & MP & 50 & -- & 2.06 & $106-44-5$ & Disinfectant. \\
\hline 4-Nonylphenol ${ }^{6}$ & NP & 100 & K & 5.99 & $84852-15-3$ & Surfactant metabolite. \\
\hline 4-Nonylphenolmonoethoxylate ${ }^{6}$ & NP1EO & 50 & $\mathrm{~K}$ & -- & -- & Surfactant metabolite. \\
\hline 4-Nonylphenoldiethoxylate ${ }^{6}$ & NP2EO & 50 & $\mathrm{~K}$ & -- & -- & Surfactant metabolite. \\
\hline 4-Nonylphenoltriethoxylate & NP3EO & 50 & $\mathrm{~K}$ & -- & -- & Surfactant metabolite. \\
\hline 4-Nonylphenoltetraethoxylate & NP4EO & 50 & $\mathrm{~K}$ & -- & -- & Surfactant metabolite. \\
\hline 4-n-Octylphenol ${ }^{6}$ & NOP & 5 & $\mathrm{~K}$ & 5.50 & $1806-26-4$ & Plasticizer. \\
\hline 4-tert-Butylphenol & ТВP & 5 & -- & 3.42 & $98-54-4$ & Antioxidant. \\
\hline 4-tert-Octylphenol ${ }^{6}$ & TOP & 5 & $\mathrm{~K}$ & 5.28 & $140-66-9$ & Surfactant metabolite. \\
\hline 4-tert-Octylphenolmonoethoxylate ${ }^{6}$ & OP1EO & 5 & $\mathrm{~K}$ & -- & -- & Surfactant metabolite. \\
\hline 4-tert-Octylphenoldiethoxylate ${ }^{6}$ & OP2EO & 5 & $\mathrm{~K}$ & & -- & Surfactant metabolite. \\
\hline 4-tert-Octylphenoltriethoxylate & OP3EO & 5 & $\mathrm{~K}$ & -- & -- & Surfactant metabolite. \\
\hline 4-tert-Octylphenoltetraethoxylate & OP4EO & 5 & $\mathrm{~K}$ & -- & -- & Surfactant metabolite. \\
\hline 4-tert-Octylphenolpentaethoxylate & OP5EO & 5 & $\mathrm{~K}$ & -- & -- & Surfactant metabolite. \\
\hline 4-tert-Pentylphenol & TPP & 5 & -- & 3.91 & $80-46-6$ & Plasticizer. \\
\hline 4-Propylphenol & $\mathrm{PP}$ & 5 & -- & 3.04 & $645-56-7$ & Plasticizer. \\
\hline 5-methyl-1H-benzotriazole ${ }^{6}$ & $5, \mathrm{MBNZ}$ & 5 & -- & 1.71 & $136-85-6$ & Antioxidant in antifreeze and deicers \\
\hline Bisphenol A ${ }^{6}$ & BPA & 5 & K & 3.64 & $80-05-7$ & FR, manuf. polycarbonate resins, antioxidant. \\
\hline Caffeine $^{6}$ & CAFF & 50 & -- & .16 & $58-08-2$ & Stimulant. \\
\hline Cholesterol $^{6}$ & $\mathrm{CHO}$ & 5 & -- & 8.74 & $57-88-5$ & Animal steroid \\
\hline Diphenhydramine & DIPH & 5 & -- & -- & -- & Pharmaceutical \\
\hline $\mathrm{N}, \mathrm{N}$, diethyl-meta-toluamide ${ }^{6}$ & DEET & 50 & -- & 2.26 & $134-62-3$ & I, urban uses, mosquito repellent. \\
\hline Triclosan $^{6}$ & TRIC & 5 & $S$ & 4.66 & $3380-34-5$ & Disinfectant, antimicrobial. \\
\hline D6-Bisphenol A (percent) & D6 BPA & -- & -- & -- & $86588-58-1$ & Surrogate standard. \\
\hline
\end{tabular}


Table 4. Physical properties and chemicals analyzed for in water samples.-Continued

[MRL, minimum reporting level; EDP, endocrine-disrupting potential; $K_{o w}$, octanol-water partition coefficient; CASRN, Chemical Abstracts Services Registry Number; $\mu$ S/cm, microsiemens per centimeter at 25 degrees Celsius $\left({ }^{\circ} \mathrm{C}\right)$; FIELD, field sample; mg/L, milligrams per liter; --, not available; NWQL, National Water Quality Laboratory; USGS-NRP, U.S. Geological Survey National Research Program (laboratory); EDTA, ethylenediaminetetraacetic acid; NTA; nitrilotriacetic acid; NPEC, nonlyphenol ethoxycarboxylate; $\mu \mathrm{g} / \mathrm{L}$, micrograms per liter; ng/L, nanograms per liter; S, suspected; K, known; E or e, remark code estimated concentration reported; F, fungicide; H, herbicide; I, insecticide; GUP, general-use pesticide; FR, flame retardant; WW, wastewater; manuf., manufacturing; \%, percent; >, greater than; CP, combustion product; PAH, polycyclic aromatic hydrocarbon; UV, ultraviolet; NA, not applicable]

\begin{tabular}{|c|c|c|c|c|c|c|}
\hline Property/chemical name & Abbreviation & MRL' & EDP2 $^{2}$ & $\log K_{o w}{ }^{3}$ & CASRN $^{4}$ & Possible compound uses or sources ${ }^{5}$ \\
\hline \multicolumn{7}{|c|}{ USGS-NRP wastewater indicators (ng/L)—Continued } \\
\hline D21-2,6-Di-tert-butyl-4-methylphenol (percent) & D21BHT & -- & -- & -- & $64502-99-4$ & Surrogate standard. \\
\hline 4- $n$-Nonylphenol (percent) & $\mathrm{nNP}$ & -- & -- & -- & $84852-15-3$ & Surrogate standard. \\
\hline 4- $n$-Nonylphenolmonoethoxylate (percent) & nNP1EO & -- & -- & -- & -- & Surrogate standard. \\
\hline 4- $n$-Nonylphenoldiethoxylate (percent) & nNP2EO & -- & -- & -- & -- & Surrogate standard. \\
\hline D4-17- $\beta$-Estradiol (percent) & D4 E2 & -- & -- & -- & $66789-03-5$ & Surrogate standard. \\
\hline D7-Cholesterol (percent) & D7 CHO & -- & -- & -- & -- & Surrogate standard. \\
\hline \multicolumn{7}{|c|}{ USGS-NWQL schedule 4433 wastewater indicators $(\mu \mathrm{g} / \mathrm{L})$} \\
\hline 1,4-Dichlorobenzene ${ }^{6}$ & $1,4 \mathrm{DCB}$ & $0.5 \mathrm{E}$ & $\mathrm{S}$ & 3.28 & $106-46-7$ & Moth repellent, fumigant, deodorant. \\
\hline 1-Methylnaphthalene & -- & $.5 \mathrm{E}$ & -- & 3.72 & $90-12-0$ & $2-5 \%$ of gasoline, diesel fuel, or crude oil. \\
\hline $2,2^{\prime}, 4,4^{\prime}$-Tetrabromodiphenyl ether & -- & .5 & -- & 6.77 & $5436-43-1$ & Widely used brominated flame retardant. \\
\hline 2,6-Dimethylnaphthalene & -- & $.5 \mathrm{E}$ & -- & 4.26 & $581-42-0$ & Percent in diesel/kerosene (trace in gasoline). \\
\hline 2-Methylnaphthalene & -- & $.5 \mathrm{E}$ & -- & 3.72 & $91-57-6$ & $2-5 \%$ of gasoline, diesel fuel, or crude oil. \\
\hline 3,4-Dichlorophenyl isocyanate & -- & $1 \mathrm{E}$ & -- & 3.88 & $102-36-3$ & Degradate of diuron, a noncrop herbicide. \\
\hline 3-beta-Coprostanol ${ }^{6}$ & $\mathrm{COP}$ & $2 \mathrm{e}$ & -- & 8.82 & $360-68-9$ & Animal fecal steroid. \\
\hline 3-Methyl-1H-indole (skatol) & -- & $1 \mathrm{e}$ & -- & 2.60 & $83-34-1$ & Fragrance, stench in feces, and coal tar. \\
\hline 3-tert-Butyl-4-hydroxyanisole ${ }^{6}$ & BHA & $5 \mathrm{E}$ & $\mathrm{S}$ & 3.50 & $25013-16-5$ & Antioxidant. \\
\hline 4-Cumylphenol & -- & 1.0 & $\mathrm{~K}$ & 4.12 & $599-64-4$ & Surfactant metabolite. \\
\hline 4-Methylphenol & MP & 1.0 & -- & 2.06 & $106-44-5$ & Disinfectant. \\
\hline 4-Nonylphenol ${ }^{6}$ & $\mathrm{NP}$ & $5 \mathrm{E}$ & $\mathrm{K}$ & 5.99 & $84852-15-3$ & Surfactant metabolite. \\
\hline 4-Nonylphenolmonoethoxylate ${ }^{6}$ & NP1EO & $2 \mathrm{E}$ & $\mathrm{K}$ & -- & -- & Surfactant metabolite. \\
\hline 4-Nonylphenoldiethoxylate ${ }^{6}$ & NP2EO & $5 \mathrm{E}$ & $\mathrm{K}$ & -- & -- & Surfactant metabolite. \\
\hline 4- $n$-Octylphenol ${ }^{6}$ & NOP & 1 & $\mathrm{~K}$ & 5.50 & $1806-26-4$ & Surfactant metabolite. \\
\hline 4-tert-Octylphenol ${ }^{6}$ & TOP & 1 & $\mathrm{~K}$ & 5.28 & $140-66-9$ & Surfactant metabolite. \\
\hline 4-tert-Octylphenolmonoethoxylate ${ }^{6}$ & OP1EO & $1 \mathrm{E}$ & $\mathrm{K}$ & -- & -- & Surfactant metabolite. \\
\hline 4-tert-Octylphenoldiethoxylate ${ }^{6}$ & OP2EO & $1 \mathrm{E}$ & $\mathrm{K}$ & -- & -- & Surfactant metabolite. \\
\hline 5-Methyl-1H-benzotriazole ${ }^{6}$ & $5, \mathrm{MBNZ}$ & $2 \mathrm{e}$ & -- & 1.71 & $136-85-6$ & Antioxidant in antifreeze and deicers. \\
\hline Acetophenone & -- & .5 & -- & 1.67 & $98-86-2$ & Fragrance and flavor. \\
\hline $\begin{array}{l}\text { Acetyl-hexamethyl-tetrahydronaphthalene } \\
\text { (tonalide) }\end{array}$ & AHTN & .5 & -- & 6.35 & $21145-77-7$ & Musk fragrance. \\
\hline Anthracene & & .5 & -- & 4.35 & $120-12-7$ & $\mathrm{CP}$, component of tar, diesel, or crude oil. \\
\hline Anthraquinone & -- & .5 & -- & 3.34 & $84-65-1$ & Manuf. dye/textiles, seed treatment, bird repellent. \\
\hline Atrazine & -- & 1e & $\mathrm{K}$ & 2.82 & $1912-24-9$ & Selective triazine herbicide. \\
\hline Benzo[a]pyrene & -- & .5 & $\mathrm{~K}$ & 6.11 & $50-32-8$ & $\mathrm{CP}$, regulated $\mathrm{PAH}$. \\
\hline
\end{tabular}


Table 4. Physical properties and chemicals analyzed for in water samples.-Continued

[MRL, minimum reporting level; EDP, endocrine-disrupting potential; $K$, octanol-water partition coefficient; CASRN, Chemical Abstracts Services Registry Number; $\mu$ S/cm, microsiemens per centimeter at 25 degrees Celsius ( $\left.{ }^{\circ} \mathrm{C}\right)$; FIELD, field sample; mg/L, milligrams per liter; --, not available; NWQL, National Water Quality Laboratory; USGS-NRP, U.S. Geological Survey National Research Program (laboratory); EDTA, ethylenediaminetetraacetic acid; NTA; nitrilotriacetic acid; NPEC, nonlyphenol ethoxycarboxylate; $\mu \mathrm{g} / \mathrm{L}$, micrograms per liter; ng/L, nanograms per liter; S, suspected; K, known; E or e, remark code estimated concentration reported; F, fungicide; H, herbicide; I, insecticide; GUP, general-use pesticide; FR, flame retardant; WW, wastewater; manuf., manufacturing; \%, percent; >, greater than; CP, combustion product; PAH, polycyclic aromatic hydrocarbon; UV, ultraviolet; NA, not applicable]

\begin{tabular}{|c|c|c|c|c|c|c|}
\hline Property/chemical name & Abbreviation & MRL'1 & EDP2 $^{2}$ & $\log K_{o w}{ }^{3}$ & CASRN $^{4}$ & Possible compound uses or sources ${ }^{5}$ \\
\hline \multicolumn{7}{|c|}{ USGS-NWQL schedule 4433 wastewater indicators $(\mu \mathrm{g} / \mathrm{L})$-Continued } \\
\hline Benzophenone & -- & 0.5 & $\mathrm{~S}$ & 3.15 & $119-61-9$ & Fixative for perfumes and soaps. \\
\hline beta-Sitosterol & -- & $2 \mathrm{E}$ & -- & 9.65 & $83-46-5$ & Plant sterol. \\
\hline beta-Stigmastanol & -- & $2 \mathrm{e}$ & -- & 9.73 & $19466-47-8$ & Herbivore fecal indicator (digestion of sitosterol). \\
\hline Bisphenol A ${ }^{6}$ & BPA & 1e & $\mathrm{K}$ & 3.64 & $80-05-7$ & FR, manuf. polycarbonate resins, antioxidant. \\
\hline Bromacil & -- & .5 & -- & 1.68 & $314-40-9$ & H (GUP), $>80 \%$ noncrop usage on grass/brush. \\
\hline Bromoform & -- & $.5 \mathrm{E}$ & -- & 1.79 & $75-25-2$ & WW ozination byproduct, military/explosives. \\
\hline Caffeine $^{6}$ & CAFF & .5 & -- & .16 & $58-08-2$ & Stimulant. \\
\hline Camphor & -- & .5 & -- & 3.04 & $76-22-2$ & Flavor, odorant, ointments. \\
\hline Carbaryl & -- & $.5 \mathrm{E}$ & $\mathrm{K}$ & 2.35 & $63-25-2$ & I, crop and garden uses, low persistence. \\
\hline Carbazole & -- & .5 & -- & 3.23 & $86-74-8$ & I, manuf. dyes, explosives, and lubricants. \\
\hline Chlorpyrifos & -- & .5 & $\mathrm{~K}$ & 4.66 & $2921-88-2$ & I, historically for domestic pest and termite control. \\
\hline Cholesterol $^{6}$ & $\mathrm{CHO}$ & $2 \mathrm{e}$ & -- & 8.74 & $57-88-5$ & Animal steroid. \\
\hline Cotinine & -- & $1 \mathrm{E}$ & -- & .34 & $486-56-6$ & Primary nicotine metabolite. \\
\hline Diazinon & -- & .5 & $\mathrm{~K}$ & 3.86 & $333-41-5$ & I, $>40 \%$ nonagricultural usage, ants, flies. \\
\hline Dichlorvos & -- & 1 & $\mathrm{~S}$ & .609 & $62-73-7$ & I, pet collars; naled or trichlofon degradate. \\
\hline Diethyl phthalate & DEP & .5 & $\mathrm{~K}$ & 2.65 & $84-66-2$ & Plasticizer for polymers and resins. \\
\hline Diethylhexyl phthalate & DEHP & 2 & $\mathrm{~K}$ & 8.39 & $117-81-7$ & Plasticizer for polymers and resins, pesticide inert. \\
\hline$d$-Limonene & -- & $.5 \mathrm{E}$ & -- & 4.83 & $5989-27-5$ & F, antimicrobial, antiviral, fragrance in aerosols. \\
\hline Fluoranthene & -- & .5 & -- & 4.93 & $206-44-0$ & $\mathrm{CP}$, in coal tar, asphalt (traces in gasoline or diesel fuel). \\
\hline $\begin{array}{l}\text { Hexahydrohexamethyl-cyclopenta-benzopyran } \\
\text { (galaxolide) }\end{array}$ & HHCB & $.5 \mathrm{e}$ & -- & 6.26 & $1222-05-5$ & Musk fragrance. \\
\hline Indole & -- & .5 & -- & 2.05 & $120-72-9$ & Pesticide inert ingredient, fragrance in coffee. \\
\hline Isoborneol & -- & .5 & -- & 2.85 & $124-76-5$ & Fragrance in perfumery, in disinfectants. \\
\hline Isophorone & -- & .5 & -- & 2.62 & $78-59-1$ & Solvent for lacquer, plastic, oil, silicon, resin. \\
\hline Isopropylbenzene (cumene) & -- & $.5 \mathrm{E}$ & -- & 3.45 & $98-82-8$ & Manuf. phenol/acetone, fuels and paint thinner. \\
\hline Isoquinoline & -- & .5 & -- & 2.14 & $119-65-3$ & Flavors and fragrances. \\
\hline Menthol & -- & .5 & -- & 3.38 & 89-78-1 & Cigarettes, cough drops, liniment, mouthwash. \\
\hline Metalaxyl & -- & .5 & -- & 1.70 & $57837-19-1$ & H, F (GUP), mildew, blight, pathogens, golf/turf. \\
\hline Methyl salicylate & -- & .5 & -- & 2.60 & $119-36-8$ & Liniment, food, beverage, UV-absorbing lotion. \\
\hline Metolachlor & -- & .5 & -- & 3.24 & $51218-45-2$ & H (GUP), indicator of agricultural drainage. \\
\hline $\mathrm{N}, \mathrm{N}$-diethyl-meta-toluamide ${ }^{6}$ & DEET & .5 & -- & 2.26 & $134-62-3$ & I, urban uses, mosquito repellent. \\
\hline Naphthalene & -- & $.5 \mathrm{E}$ & -- & 3.17 & $91-20-3$ & Fumigant, moth repellent, component (10\%) of gasoline. \\
\hline Pentachlorophenol & -- & $2.0 \mathrm{E}$ & S & 4.74 & $87-86-5$ & $\mathrm{H}, \mathrm{F}$, wood preservative, termite control. \\
\hline Phenanthrene & -- & .5 & -- & 4.35 & $85-01-8$ & $\mathrm{CP}$, manuf. explosives, in tar, diesel fuel, or crude oil. \\
\hline
\end{tabular}


Table 4. Physical properties and chemicals analyzed for in water samples.-Continued

[MRL, minimum reporting level; EDP, endocrine-disrupting potential; $K_{o w}$, octanol-water partition coefficient; CASRN, Chemical Abstracts Services Registry Number; $\mu$ S/cm, microsiemens per centimeter at 25 degrees Celsius $\left({ }^{\circ} \mathrm{C}\right)$; FIELD, field sample; mg/L, milligrams per liter; --, not available; NWQL, National Water Quality Laboratory; USGS-NRP, U.S. Geological Survey National Research Program (laboratory); EDTA, ethylenediaminetetraacetic acid; NTA; nitrilotriacetic acid; NPEC, nonlyphenol ethoxycarboxylate; $\mu \mathrm{g} / \mathrm{L}$, micrograms per liter; ng/L, nanograms per liter; S, suspected; K, known; E or e, remark code estimated concentration reported; F, fungicide; H, herbicide; I, insecticide; GUP, general-use pesticide; FR, flame retardant; WW, wastewater; manuf., manufacturing; \%, percent; >, greater than; CP, combustion product; PAH, polycyclic aromatic hydrocarbon; UV, ultraviolet; NA, not applicable]

\begin{tabular}{|c|c|c|c|c|c|c|}
\hline Property/chemical name & Abbreviation & MRL' & EDP $^{2}$ & $\log K_{o w}{ }^{3}$ & CASRN $^{4}$ & Possible compound uses or sources ${ }^{5}$ \\
\hline \multicolumn{7}{|c|}{ USGS-NWQL schedule 4433 wastewater indicators $(\mu \mathrm{g} / \mathrm{L})$-Continued } \\
\hline Phenol & -- & 0.5 & -- & 1.51 & $108-95-2$ & Disinfectant, manuf several products, leachate. \\
\hline Prometon & -- & .5 & -- & 3.57 & $1610-18-0$ & H (non-crop only), applied prior to blacktop. \\
\hline Pyrene & -- & .5 & -- & 4.93 & $129-00-0$ & $\mathrm{CP}$, In coal tar, asphalt (traces in gasoline or diesel fuel). \\
\hline Tetrachloroethene & PCE & $.5 \mathrm{E}$ & -- & 2.97 & $127-18-4$ & Solvent, degreaser, veterinary anthelmintic. \\
\hline Tri(2-butoxyethyl) phosphate & -- & $.5 \mathrm{e}$ & -- & 3.00 & $78-51-3$ & Flame retardant. \\
\hline Tri(2-chloroethyl) phosphate & -- & .5 & S & 1.63 & $115-96-8$ & Plasticizer, flame retardant. \\
\hline Tri(dichloroisopropyl) phosphate & -- & .5 & S & 3.65 & $13674-87-8$ & Flame retardant. \\
\hline Tributyl phosphate & -- & .5 & -- & 3.82 & $126-73-8$ & Antifoaming agent, flame retardant. \\
\hline Triclosan $^{6}$ & TRIC & 1 & $\mathrm{~S}$ & 4.66 & $3380-34-5$ & Disinfectant, antimicrobial. \\
\hline Triethyl citrate (ethyl citrate) & -- & .5 & -- & .33 & $77-93-0$ & Cosmetics, pharmaceuticals. \\
\hline Triphenyl phosphate & -- & .5 & -- & 4.70 & $115-86-6$ & FR, plasticizer, resin, wax, finish, roofing paper. \\
\hline Bisphenol A-d3 (percent) & & & & -- & -- & Surrogate standard. \\
\hline Caffeine-13C (percent) & -- & -- & & -- & -- & Surrogate standard. \\
\hline Decafluorobiphenyl (percent) & -- & -- & & -- & -- & Surrogate standard. \\
\hline Fluoranthene-d10 (percent) & -- & -- & & -- & -- & Surrogate standard. \\
\hline
\end{tabular}

${ }^{1}$ Chemicals with and "E" following the number indicate a compound with low recovery, unstable instrument response, or reference standard prepared from a technical mixture for water analyses (Zaugg and others, 2006). Chemicals with an "e" following the number are estimated if the spike recovery or expected continuing calibration verification concentrations for each set of samples are not within control limits (Zaugg and others, 2006).

${ }^{2}$ Endocrine disrupting potential (EDP) from the following sources: Kime, 1998; Tremblay and Van der Kraak, 1998; EC-BKH, 2000; Nishihara and others, 2000; Zaugg and others, 2002; Global Water Research Coalition, 2003; Versonnen and others, 2003; Institute of Environmental Health, 2005; Korner and others, 2005; and Terasaki and others, 2005.

${ }^{3} \log K_{o w}$ is the octanol-water partition coefficient and is a measure of the equilibrium concentration of a compound between octanol and water. A high value indicates a compound that will preferentially partition into soil organic matter rather than water. It was calculated using the U.S. Environmental Protection Agency's exposure assessment tools and models (EPI-suite software, WSKOWWINTM version 1.40; U.S. Environmental Protection Agency, 2005b).

${ }^{4}$ This report contains Chemical Abstracts Services Registry Numbers (CASRN) ${ }^{\oplus}$, which is a Registered Trademark of the American Chemical Society. A CASRN is a numeric identifier that can contain up to nine digits, divided by dashes into three parts. For example, 58-08-2 is the CASRN for caffeine. The online database provides a source for the latest registry number information: http://www.cas.org/. Chemical Abstracts Services recommends the verification of the CASRNs through CAS Client Services ${ }^{\mathrm{SM}}$.

${ }^{5}$ Sources are Kime, 1998; Tremblay and Van der Kraak, 1998; EC-BKH, 2000; Nishihara and others, 2000; Zaugg and others, 2002; Versonnen and others, 2003; Barber, Furlong, and others, 2003; Global Water Research Coalition, 2003; Institute of Environmental Health, 2005; Korner and others, 2005; Terasaki and others, 2005.

${ }^{6}$ Analyzed using both the USGS-NWQL and USGS-NRP. 
Boulder, Colo., used research methods to analyze water and bed-sediment samples for APs and other wastewater indicator chemicals.

Major ions and nutrients were analyzed at the USGSNWQL using standard analytical techniques described in Fishman and Friedman (1989), Patton and Truitt (1992), Fishman (1993), U.S. Environmental Protection Agency (1993), and Fishman and others (1994). Samples analyzed for dissolved major-ion and nutrient concentrations were filtered using 0.45 - $\mu \mathrm{m}$-pore-size encapsulated filters. Nutrient samples were preserved and maintained at $4^{\circ} \mathrm{C}$ until analyzed. Samples analyzed to determine total nutrient concentrations were not filtered. Dissolved phosphorus was analyzed using U.S. Environmental Protection Agency method 365.1, low-level persulfate digestion (U.S. Environmental Protection Agency, 1993).

Water samples collected during each of the six sampling periods were analyzed for three measures of organic carbon, 6 EDTA-NTA-NPEC chemicals, 32 wastewater indicator chemicals (including APs, APEOs, antimicrobials, plastic components, and sterols), and 8 surrogate standards (table 4) at the USGS-NRP as described in Barber and others (2000); Barber, Furlong, and others (2003); and Barber, Keefe, and others (2003). Three measures of organic carbon were used for this study: (1) TOC, (2) UV absorbance at a wavelength of 254 nanometers $(\mathrm{nm})\left(\mathrm{UV}_{254}\right)$, and (3) specific $\mathrm{UV}$ absorbance at a wavelength of $254 \mathrm{~nm}\left(\mathrm{SUVA}_{254}\right)$. $\mathrm{UV}_{254}$ is a surrogate measure of organic content and is the absorbance of UV light at a wavelength of $254 \mathrm{~nm}$. SUVA 254 is the UV absorbance of a water sample at a wavelength of $254 \mathrm{~nm}$ normalized for TOC; it is useful for estimating aromatic carbon content in aquatic environments (Barber and others, 2001; Weishaar and others, 2003). Details of the organic carbon analytical methods are reported elsewhere (Barber and others, 2001; Barber, Furlong, and others 2003; Barber, Keefe and others, 2003). Briefly, TOC was measured by persulfate oxidation, with infrared detection by using an Oceangraphics International model 700 carbon analyzer. UV light absorbance of the filtered samples was measured at $254 \mathrm{~nm}$ in a 1-centimeter (cm)-light-path quartz cell using a Spectronics/Unicam Genesys model 10UVspectrometer.

EDTA, nitrilotriacetic acid (NTA), and nonylphenolmonoethoxycarboxylate to nonylphenoltetraethoxycarboxylate (NP1EC-NP4EC) were measured using a modification (Barber and others, 2000; Barber, Furlong, and others 2003; Barber, Keefe, and others, 2003) of the method of Schaffner and Giger (1984). Samples (100 milliliters (mL)) were acidified with $5 \mathrm{~mL}$ of 50 percent (volume/volume) formic acid/distilled water, and evaporated to dryness. Acetyl chloride/propanol (10 percent volume/volume) was added, the sample was heated at $90^{\circ} \mathrm{C}$ for 1 hour to form the propyl esters, and the propyl esters were extracted into chloroform. The chloroform extracts were evaporated to dryness and redissolved in toluene for analysis by gas chromatography-mass spectrometry as described in the following.

NP and other wastewater indicator chemicals were measured as described in Barber and others (2000), Barber,
Furlong, and others (2003), and Barber, Keefe, and others (2003). This method uses continuous liquid-liquid extraction (CLLE) with methylene chloride at $\mathrm{pH}$ 2. The CLLE exposes the sample to methylene chloride by refluxing and dispersing the solvent through a coarse glass frit, thus forming microdroplets that travel an extended path through the sample matrix allowing effective partitioning of the wastewater compounds into the solvent. After extraction, the solvent was dried over sodium sulfate and the volume reduced to 500 microliters $(\mu \mathrm{L})$ under a stream of nitrogen for gas chromatography-mass spectrometry analysis.

The propyl-ester and CLLE extracts were analyzed by electron impact gas chromatography-mass spectrometry in both the full-scan and selected-ion-monitoring modes. The general gas chromatography conditions were as follows: Hewlett Packard (HP) 6890 gas chromatography; columnHP Ultra II (5 percent phenylmethyl silicone), 25 meters (m) by 0.2 millimeters (mm), 33- $\mu \mathrm{m}$ film thickness; carrier gas, ultra-high-purity helium with a linear flow velocity of 27 centimeters per second $(\mathrm{cm} / \mathrm{s})$; injection port temperature, $300^{\circ} \mathrm{C}$; initial oven temperature, $50^{\circ} \mathrm{C}$; split vent open, 0.75 minutes; ramp rate, $6^{\circ} \mathrm{C} /$ minute to $300^{\circ} \mathrm{C}$; and hold time, 15 minutes at $300^{\circ} \mathrm{C}$. The mass spectrometer conditions were as follows: HP 5973 Mass Selective Detector; tune with perflurotributylamine; ionization energy, 70 electrovolts; source pressure, $1 \times 10^{-5}$ torr; source temperature, $250^{\circ} \mathrm{C}$; interface temperature, $280^{\circ} \mathrm{C}$; full scan, 40 to 550 atomic mass units (amu) at 1 scan per second.

Concentrations were calculated on the basis of selectedion-monitoring data using diagnostic ions for each compound. Each compound was identified on the basis of matching of retention times (plus or minus 0.02 minutes) and ion ratios (plus or minus 20 percent) determined from analysis of authentic standards. An 8-point calibration curve (typically ranging from 0.01 to $50 \mathrm{ng} / \mu \mathrm{L}$ ) and internal standard (deuterated polycyclic aromatic hydrocarbons) procedures were used for calculating concentrations. Surrogate standards were added to the samples before extraction and derivatization to evaluate compound recovery and whole method performance. Detection limits were established using multiple qualityassurance data including the lowest concentration detectable on a standard curve, spike and recovery data, and occurrence in laboratory blanks.

Water samples collected during two time periods (May and August 2007) were analyzed at the USGS-NWQL for 69 wastewater indicator chemicals typically found in domestic and industrial wastewater and nonpoint sources and 4 surrogate chemicals by using the laboratory production schedule 4433 (Zaugg and others, 2006; table 4). These chemicals include the alkylphenol ethoxylate nonionic surfactants, food additives, fragrances, antioxidants, flame retardants, plasticizers, industrial solvents, disinfectants, fecal sterols, polycyclic aromatic hydrocarbons, and high-use domestic pesticides. Whole-water samples were extracted using CLLE with methylene chloride solvent and measured by gas chromatographymass spectrometry. Samples were preserved by adding 
60 grams $(\mathrm{g})$ of sodium chloride and stored at $4{ }^{\circ} \mathrm{C}$. The holding-time limit prior to sample extraction was 14 days from the date of collection. The alkylphenol monoethoxylates (AP1EOs) are represented in the method by 4-octylphenolmonoethoxylate (OP1EO) and NP1EO (total of numerous isomers). The alkylphenol diethoxylates are represented in the method by 4-octylphenoldiethoxylate (OP2EO) and NP2EO (total of numerous isomers). The APs are represented in the method by 4-cumylphenol, 4- $n$-octylphenol, NP (total), and 4-tert-octylphenol (TOP).

The analytical method for NWQL schedule 4433 is considered to be information rich because chemical identifications are determined by mass spectrometry; consequently, results are not censored at the minimum reporting level (MRL) (Childress and others, 1999). The MRL is the smallest measured concentration of a constituent that may be reliably reported by using a given analytical method (Timme, 1995). The intention is to produce as much information as possible for complex samples, but it is difficult to consistently report concentrations near the method detection limit (MDL). The MDL is the minimum concentration that can be measured and reported with a 99-percent confidence that the concentration is greater than zero (U.S. Environmental Protection Agency, 2002). The MRLs are set higher than the calculated MDLs as a precaution to reduce the risk of reporting false positives (Zaugg and others, 2006). Chemical concentrations for NWQL schedule 4433 analyses are reported as follows. If concentrations are equal to or greater than the MRL or the lowest calibration standard $(0.2 \mu \mathrm{g} / \mathrm{L})$, then results are reported to three significant figures. If the concentrations are less than the MRL or the lowest calibration standard, then the results are reported as estimated, using an "E" code. Reporting chemical results as estimated because their concentrations are less than the MRL does not decrease confidence in qualitative identification of a chemical. However, there is more uncertainty for concentrations reported near or less than the MDL (Zaugg and others, 2006).

Water samples collected daily from the Redwood River near Marshall, Minn. (USGS station 05315000) for a timeseries investigation were analyzed at the USGS-NRP for total APs and atrazine by enzyme-linked immunosorbent assay (ELISA) kits developed by Japan EnviroChemicals (APs) and Abraxis (atrazine). The total AP ELISA method is specific for NP and octylphenol, and it shows less than a 5 percent response to the other AP homologues (alkylphenolethoxylates and APECs). Approximately $100 \mathrm{~mL}$ of the water sample was concentrated by solid-phase extraction on a Supelco ENVI-18 column (glass, $500 \mathrm{mg}$ ). The column was first conditioned with $3 \mathrm{~mL}$ of dichloromethane followed by $6 \mathrm{~mL}$ of methanol. The sample was then applied to the column at a rate of 1-5 $\mathrm{mL}$ per minute, dried under vacuum for 30 minutes, and eluted with $6 \mathrm{~mL}$ of methanol. The methanol was evaporated under a stream of nitrogen gas to a final volume of $1.0 \mathrm{~mL}$. Following procedures outlined by the manufacturers of each kit, concentrations of total APs and atrazine were determined by ELISA on the concentrated $1.0 \mathrm{~mL}$ extracts. The approximate detection limit was $50 \mathrm{ng} / \mathrm{L}$ for the AP method and $1 \mathrm{ng} / \mathrm{L}$ for the atrazine method.

\section{Bed-Sediment Collection and Analyses}

Bed-sediment samples were collected during one sampling period at each of the stream locations according to established USGS protocols (U.S. Geological Survey, 2003). Samples were collected with stainless-steel sampling equipment from the top $20 \mathrm{~cm}$ of bed sediment at 5 depositional areas at each sampling location (fig. 9). The bed-sediment sample was discarded if it contained a large amount of vegetation or appeared to be disturbed; in this case, a new sample was then collected. Bed-sediment samples were sieved using a 2-mm stainless-steel sieve and transferred to a glass container and homogenized for 5 minutes. Two-hundred grams of sieved wet material was placed in wide-mouth glass containers and chilled prior to transfer to the USGS-NRP to be analyzed for selected chemicals (table 5).

Bed-sediment samples were analyzed for 29 chemicals and 7 surrogate standards using the methods described in Burkhardt and others (2006) for sample extraction and cleanup, followed by gas chromatography-mass spectrometry analysis (Barber and others, 2000; Barber, Furlong and others, 2003) to identify and quantify compounds. Approximately 40 grams of sediment were extracted twice by accelerated solvent extraction, first for 10 minutes by $25 \mathrm{~mL}$ of 50:50 water/isopropanol at a temperature of $120^{\circ} \mathrm{C}$ and a pressure of 13,800 kilopascals $(\mathrm{kPa} ; 2,000$ pounds per square inch (lb/in. $\left.{ }^{2}\right)$ ), followed by $25 \mathrm{~mL}$ of 20:50 water/isopropanol at a temperature of $200^{\circ} \mathrm{C}$ and a pressure of $13,800 \mathrm{kPa}$ $\left(2,000 \mathrm{lb} / \mathrm{in}^{2}\right)$. The extracts were combined and diluted with $100 \mathrm{~mL}$ of $\mathrm{pH} 7.0$ phosphate buffer, passed through an Oasis PSDVB solid-phase extraction cartridge, and eluted with $25 \mathrm{~mL}$ of 80:20 dichloromethane/diethyl ether. The Oasis extract was then cleaned by passing it through a Fluorisil solid-phase extraction cartridge containing sodium sulfate. The final extract was reduced in volume to $1.0 \mathrm{~mL}$ and then analyzed by gas chromatography-mass spectrometry in the full-scan and selected-ion-monitoring modes as described previously for the CLLE extracts. Quality assurance for the sediment analysis consisted of laboratory blanks, clean sand matrix spikes, duplicate environmental sample analysis, and surrogate standards.

\section{Fish Collection and Analyses}

Common carp (Cyprinus carpio), common shiner (Luxilus cornutus), creek chub (Semotilus atromaculatus), fathead minnow (Pimephales promelas), redhorse (Moxostoma ssp.), and white sucker (Catostomus commersonii) were selected for sampling on the basis of their known occurrence in the rivers studied, the variety of feeding and reproductive strategies filled by these species, and use in previous research. All fish in this study are considered opportunistic omnivores (Eddy 

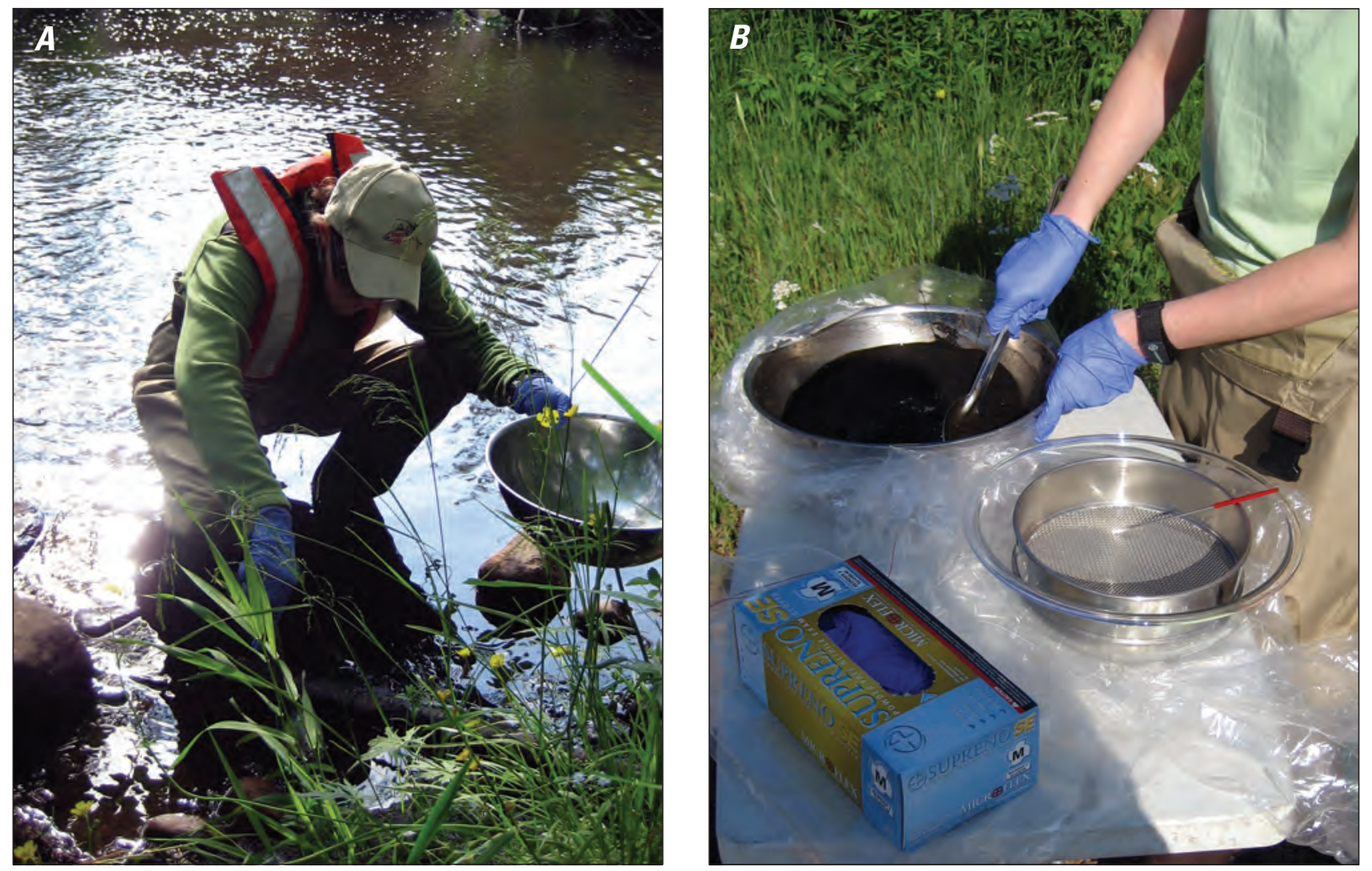

Figure 9. Bed-sediment sample A, collection, and B, processing at the Grindstone River, June 2007.

and Underhill, 1974; Becker, 1983). Common carp, redhorse, and white sucker are benthic feeders, whereas common shiner, creek chub, and fathead minnow are pelagic feeders (Eddy and Underhill; 1974; Becker, 1983).

Fish that migrate may not indicate local conditions. However, all three streams had small dams located upstream from the WWTP, which should have precluded migration from the WWTP sites to the upstream sites in all streams in this study. Large fish in this study tend to migrate greater distances than the smaller fish. Carp generally do not migrate distances greater than 2 miles (although some have been known to travel hundreds of miles) (Funk, 1955; Becker, 1983). Redhorse and carp have migrated as much as 10 miles (Olson and Scidmore, 1963; Hackney and others, 1968) during spawning. Creek chub (Becker, 1983) but do not appear to migrate distances greater than 100 feet to spawn (Copes, 1978). Small downstream migration of fathead minnow may occur during high water (Schlosser, 1995), whereas common shiner do not appear to migrate large distances (Miller, 1964).

All fish were collected between May 17 and July 6, 2007, by using electrofishing techniques (fig. 10, table 6). Fish were netted and kept alive in an aerated cooler for approximately 2 hours until they were anesthetized in 1 percent clove oil. Fish were weighed and total and standard lengths were recorded (fig. 11). Condition factors were calculated as

$$
\frac{\text { Weight }(\text { in grams })}{\text { Total length }^{3}(\text { in cubic millimeters })} \times 100,000 .
$$

Blood was drawn with a needle and syringe from the caudal vasculature in large fish and by severing the tail and applying a capillary tube to the caudal vein in small fish. Blood was stored in hematocrit vials (Phoenix Research Products, Hayward, Calif.) on ice and was returned to the laboratory within

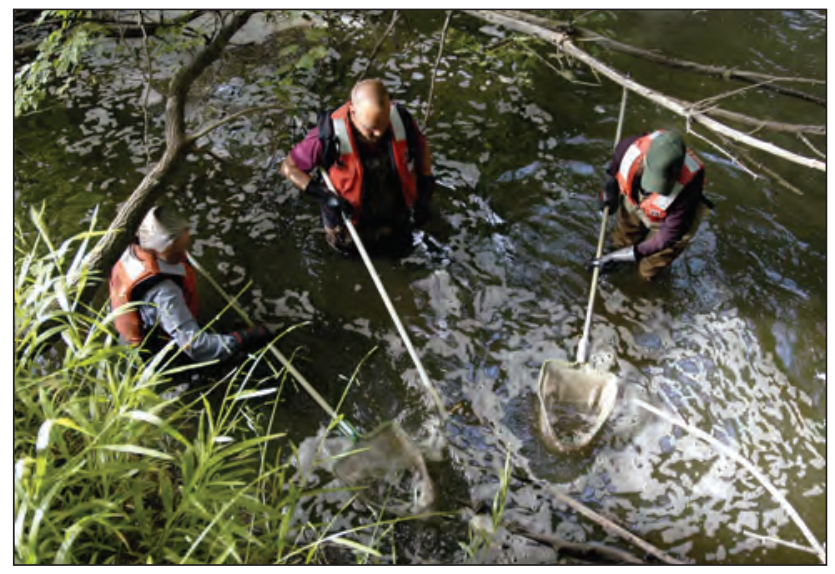

Figure 10. Fish collection using electrofishing techniques. 
Table 5. Chemicals for which bed-sediment samples were analyzed at the U.S. Geological Survey National Research Program Laboratory.

[MRL, minimum reporting level; ng/g, nanogram per gram; $K_{o w}$, octanol-water partition coefficient; CASRN, Chemical Abstracts Services Registry Number; --, no data].

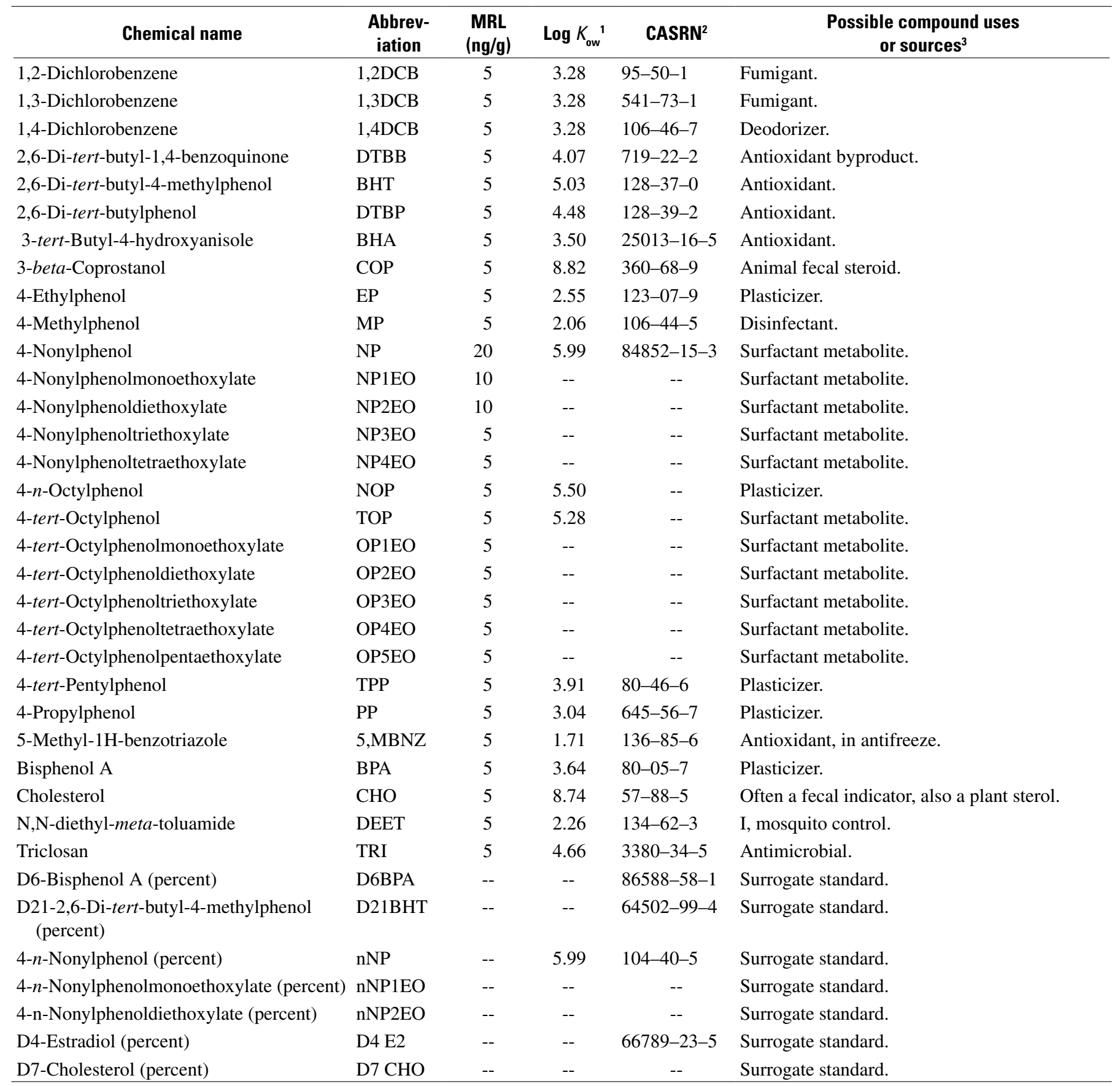

${ }^{1} \log K_{o w}$ is the octanol-water partition coefficient and is a measure of the equilibrium concentration of a compound between octanol and water. A high value indicates a compound that will preferentially partition into soil organic matter rather than water. It was calculated using U.S. Environmental Protection Agency's exposure assessment tools and models (EPI-suite software, WSKOWWINTM version 1.40; U.S. Environmental Protection Agency, 2005b).

${ }^{2}$ This report contains Chemical Abstracts Services Registry Numbers (CASRN) ${ }^{\circledR}$, which is a Registered Trademark of the American Chemical Society. A CASRN is a numeric identifier that can contain up to nine digits, divided by dashes into three parts. For example, 58-08-2 is the CASRN for caffeine. The online database provides a source for the latest registry number information: $h t t p: / / w w w . c a s . o r g /$. Chemical Abstracts Services recommends the verification of the CASRNs through CAS Client Services ${ }^{\mathrm{SM}}$.

${ }^{3}$ Sources are Kime, 1998; Tremblay and Van der Kraak, 1998; EC-BKH, 2000; Nishihara and others, 2000; Zaugg and others, 2002; Versonnen and others, 2003; Barber, Furlong, and others, 2003; Global Water Research Coalition, 2003; Institute of Environmental Health, 2005; Korner and others, 2005; Terasaki and others, 2005. 
Table 6. Numbers of fish collected by St. Cloud State University.

\begin{tabular}{|c|c|c|c|c|c|c|c|}
\hline \multirow[b]{2}{*}{$\begin{array}{c}\text { Site } \\
\text { abbreviation }\end{array}$} & \multirow[b]{2}{*}{$\begin{array}{c}\text { Date } \\
\text { sampled }\end{array}$} & \multicolumn{6}{|c|}{ Number of fish collected ${ }^{1}$} \\
\hline & & $\begin{array}{l}\text { Common carp } \\
\text { (Cyprinus } \\
\text { carpio) }\end{array}$ & $\begin{array}{c}\text { Common shiner } \\
\text { (Luxilus } \\
\text { cornutus) }\end{array}$ & $\begin{array}{c}\text { Creek chub } \\
\text { (Semotilus } \\
\text { atromaculatus) }\end{array}$ & $\begin{array}{c}\text { Fathead minnow } \\
\text { (Pimephales } \\
\text { promelas) }\end{array}$ & $\begin{array}{l}\text { Redhorse ssp. } \\
\text { (Moxostoma) }\end{array}$ & $\begin{array}{l}\text { White sucker } \\
\text { (Catostomus } \\
\text { commersonit) }\end{array}$ \\
\hline SFCROW-US1 & $6 / 8 / 2007,6 / 22 / 2007$ & $7(2)$ & $0(0)$ & $0(0)$ & $29(3)$ & $0(0)$ & $1(2)$ \\
\hline $\begin{array}{l}\text { SFCROW AT } \\
\text { WWTP }\end{array}$ & $6 / 11 / 2007,7 / 2 / 2007$ & $14(5)$ & $9(3)$ & $0(0)$ & $22(2)$ & $7(7)$ & $3(2)$ \\
\hline REDWD-US1 & $6 / 5 / 2007,7 / 5 / 2007$ & $0(0)$ & $15(9)$ & $13(12)$ & $7(1)$ & $6(11)$ & $4(6)$ \\
\hline $\begin{array}{l}\text { REDWD AT } \\
\text { WWTP }\end{array}$ & $6 / 18 / 2007,7 / 5 / 2007$ & $1(2)$ & $21(12)$ & $6(12)$ & $10(3)$ & $10(12)$ & $6(9)$ \\
\hline REDWD-DS1 & 6/19/2007, 7/6/2007 & $3(7)$ & $19(9)$ & $9(9)$ & $0(0)$ & $9(17)$ & $0(9)$ \\
\hline REDWD-DS2 & 6/19/2007, 7/6/2007 & $6(9)$ & $14(7)$ & $5(4)$ & $2(1)$ & $7(12)$ & $4(3)$ \\
\hline GRIND-DS1 & $5 / 29 / 2070,6 / 27 / 2007$ & $0(0)$ & $9(25)$ & $1(0)$ & $0(1)$ & $1(1)$ & $6(1)$ \\
\hline GRIND-DS2 & $5 / 30 / 2007$ & $0(0)$ & $8(16)$ & $4(1)$ & $1(0)$ & $1(0)$ & $4(6)$ \\
\hline
\end{tabular}

${ }^{1}$ Number of male fish shown outside parenthesis; number of female fish shown inside parenthesis.

24 hours for plasma separation (centrifuged at 3,500 revolutions per minute for 5 minutes and stored at $\left.-80^{\circ} \mathrm{C}\right)$. After blood sampling, fish were sacrificed with clove oil. Livers were removed from all fish and gonads were removed from male fish as male fish responses were the focus of this study. Gonad and liver samples from each individual fish were placed in histocassettes and stored in 10 percent buffered formalin (fig. 12). Livers and gonads of larger fish (greater than approximately $200 \mathrm{~g}$ ) were weighed.

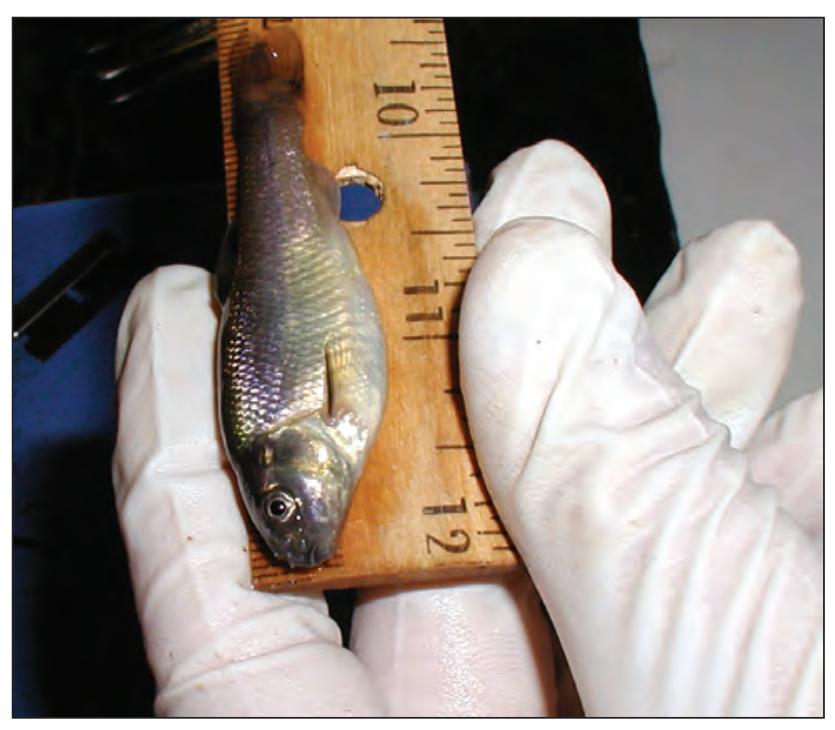

Figure 11. Fish length measurement.
The gonads and livers of small fish (less than 7 ounces or $200 \mathrm{~g}$ ) were not weighed because accurate measurements were not possible in the field. Gonadosomatic indexes (GSIs) were calculated by the following (Allen and others, 1999):

$$
G S I=\frac{\text { Gonad weight }(\text { in grams })}{\text { Body weight }(\text { in grams })} \times 100 .
$$

Hepatosomatic indexes (HSIs) were calculated by the following (Allen and others, 1999):

$$
H S I=\frac{\text { Liver weight }(\text { in grams })}{\text { Body weight }(\text { in grams })} \times 100 .
$$

All samples were transported to the Aquatic Toxicology Laboratory at St. Cloud State University within 24 hours for analyses following established and previously published protocols (Bistodeau and others, 2006; Barber and others, 2007; Schoenfuss and others, 2008) and guidelines established by the U.S. Environmental Protection Agency (2006b).

An indirect competitive fathead minnow immunoassay was used to assess Vtg in all male fish following Zhang and others (2004). Wells in each plate were coated with fathead minnow Vtg at an approximate concentration of $4 \mu \mathrm{g} / \mathrm{mL}$ (stock Vtg $3.2 \mathrm{mg} / \mathrm{mL}$ diluted 1:800) in coating buffer (0.5 molar carbonate buffer, $\mathrm{pH}$ 9.6). The dilution of the $\mathrm{Vtg}$ standards and both the primary and secondary antibody dilutions were optimized through titration prior to assay performance. One well on each plate was coated with 5 percent bovine serum albumin in coating buffer for a nonspecific binding subtraction. Samples and standards were diluted and 


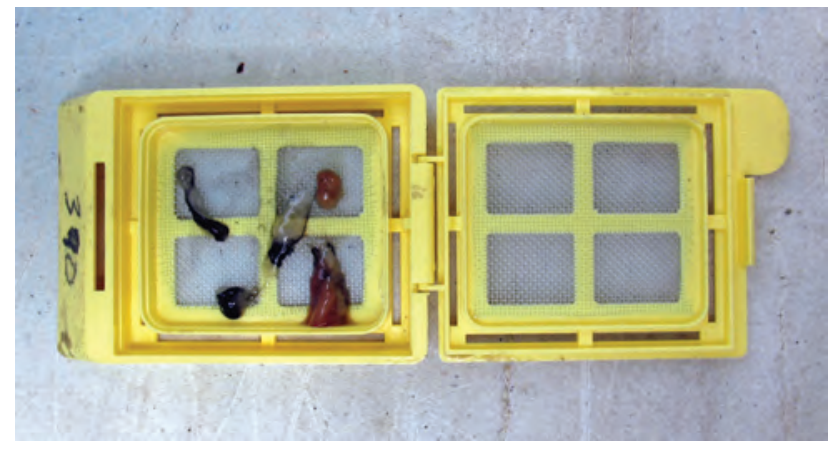

Figure 12. Histological cassette containing testes and liver tissue.

mixed 1:1 with a polyclonal antifathead minnow Vtg antibody (final dilution 1:20,000) (provided by Gerald LeBlanc, North Carolina State University) and incubated at $37^{\circ} \mathrm{C}$ for 2 hours. Plates were washed three times in an automated plate washer, standards were added to the wells $(200 \mu \mathrm{L})$, and plates were incubated for 1 hour at room temperature. Following this preincubation period, plates were triple washed and probed with a horseradish peroxidase labeled antirabbit secondary antibody (Sigma-Aldrich, St. Louis, Mo.) at a dilution of 1:10,000 for 1 hour at room temperature. Plates were washed with $200 \mu \mathrm{L}$ of 0.3 ',5.5'-tetramethylbenzidine substrate and incubated at room temperature in the dark for 20 minutes. Absorbencies were read at 620 nm on a Thermo Multiskan EX microplate reader (Vantaa, Finland). Ascent Software version 2.6 was used for logistic regression of the standards and calculation of the sample Vtg quantities.

The polyclonal antifathead minnow Vtg antibody used in the preincubation step was used to bind Vtg in either the sample or standard to decrease the likelihood that Vtg would be available to bind to the coating in the wells. A concern with this type of assay is the possibility of bias due to affinity of the antibody for the coated Vtg instead of the sample Vtg. Because ELISA generally is an equilibrium assay and antibody binding is reversible, higher affinity of the antibody for one type of Vtg rather than another would influence results. The antibody is a rabbit polyclonal antiserum produced against semipurified fathead minnow Vtg. Because the antifathead minnow $\mathrm{Vtg}$ assay is polyclonal, many recognized epitopes are likely. It is possible that the binding affinity of Vtg varies among certain fish. However, fish species selected in this study are within the two families of cyprinidae and catostomidae (all in order Cypriniformes), which may raise the probability that the identical recognizable epitopes of $\mathrm{Vtg}$ are present in all species of fish tested, thereby negating large affinity differences. The standard curves generated from Vtg standards did not contain large zero-slope areas indicative of maximal binding to the coated antigen. This indicates that the polyclonal antifathead minnow Vtg antibody is capable of recognizing the Vtg epitopes in selected fish and remain-

ing bound (that is, not pulled off by higher affinity for the coated antigen). The assay is very robust, and $\mathrm{R}^{2}$ (coefficient of determination) values of diluted fathead minnow Vtg routinely are in the 0.97-0.99 range. Plasma samples were analyzed in triplicate for $\mathrm{Vtg}$ concentrations. A majority of the standard curves for these samples had $\mathrm{R}^{2}$ values greater than 0.99. Standard curves were calculated on the basis of 5 to 7 dilution points (after removing the highest and lowest dilution points). The method detection limit for $\mathrm{Vtg}$ is $0.54 \mu \mathrm{g} / \mathrm{mL}$.

Gonads and livers of all male fish were removed and processed for histological analysis. Histological cassettes were processed in a Jung TP1050 automated tissue processor (Leica, Wetzlar, Germany) according to an established histological protocol of dehydration and embedding in paraffin wax (Gabe, 1976). Once embedded, histological sections (three per histological cassette) were produced and stained with hematoxylin and eosin (two sections) and reticular stains (one section). Paraffin-embedded tissue blocks were sectioned to 5- $\mu \mathrm{m}$ thickness on a Reichert-Jung 2030 microtome. Gonad and liver tissues were graded on a five-point scale according to U.S. Environmental Protection Agency (2006b). Briefly, a grade of 0 indicates absence of a cell type; a score of 1 indicates minimal presence (less than 2 percent) of a cell type; a score of 2 indicates apparent but not extensive occurrence (less than 25 percent) of a cell type; a score of three indicates a significant presence (25-50 percent) of a cell type; and a score of 5 indicates an abundant presence of a cell type (greater than 50 percent) of the entire tissue section. Testicular endpoints assessed were abundance of spermatagonia (immature sperm) and spermatozoa (intermediate sperm), Sertoli and Leydig cell hypertrophy (support cells for sperm production), and degradation of seminiferous tubules (testicular structures containing sperm). Liver stress and functionality also were assessed on a five-point scale in the form of liver adipocyte abundance using the grading scale described for the gonad and liver tissues. The slides also were examined for intersex (oocytes in testes tissue).

\section{Quality Assurance and Control}

The USGS National Field Manual (U.S. Geological Survey, 2003) was used to guide water and bed-sediment data collection. A quality-assurance plan for this study was established to evaluate field sampling and laboratory techniques for water and bed sediment, to assess possible sources of contamination, and to assure that samples representative of streamflow conditions were collected. All field personnel were familiar with study design and sampling protocols before sampling or sample processing to ensure sample integrity. Laboratory quality-control samples were used to validate analytical data, and field quality-control samples were used to validate collection and processing methods. 
Laboratory quality-control samples at the USGS-NRP included laboratory blanks, replicates, and surrogates as outlined in Barber and others $(2000,2007)$ and Barber, Keefe, and others (2003); the method is briefly described in this section. Laboratory reagent blanks were used to assess potential sample contamination in the laboratory. None of the chemicals analyzed were detected in any of the laboratory reagent blank samples.

Surrogate standards are chemicals that have properties similar to those of the analytes of interest, but they do not interfere with quantification of the chemicals of interest. One surrogate standard (4- $n$-NP1EC) was added to environmental samples analyzed for EDTA, NTA, and NPECs at the USGS-NRP (table 4). Seven surrogate standards were added to environmental samples for analyses of APs and other wastewater indicator chemicals analyzed at the USGSNRP: D6-bisphenol A, D21-2,6-di-tert-butyl-4-methylphenol, 4-n-nonylphenol, 4- $n$-NP1EO, 4- $n$-NP2EO, D4-estradiol, and D7-cholesterol (table 4).

The average percent recovery of the surrogate 4- $n$ NP1EC, which was analyzed at the USGS-NRP with environmental samples for EDTA, NTA, and NPEC, was 119 percent with a standard deviation of plus or minus 49 percent and a range of 66 to 357 percent (appendix 1). Most of the samples with surrogate recoveries greater than 150 percent were either from effluent samples or samples collected downstream from effluent discharges with the exception of two samples collected at the upstream site on the South Fork Crow River (SFCROW-US1) at two different times (February 28 and May 9, 2007).

The average percent recoveries for D21-2,6-di-tert-butyl4-methylphenol and D7-cholesterol were consistently lower (averages of 13 and 16 percent, respectively) than the other five surrogate standards analyzed (average of 62 percent) at the USGS-NRP analyzed with study samples for APs and other wastewater indicator chemicals. A few environmental samples had low surrogate recoveries for multiple surrogates including the downstream-1 and downstream-2 sites on the South Fork Crow River (SFCROW-DS1, and SFCROW-DS2) collected February 28, 2007; the downstream-2 site on the Redwood River (REDWD-DS2) collected on May 10, 2007; the upstream site on the Redwood River (REDWD-DS1) collected on June 21, 2007; and the upstream-1 site on the Grindstone River (GRIND-US1) collected on March 7, 2007 (appendix 1).

Laboratory quality-control samples for analyses of wastewater indicators in water using schedule 4433 at the USGS-NWQL included laboratory blanks, reagent spikes, and surrogates. Full details of the method can be found in Zaugg and others (2006); the method is briefly described in this section. At least one fortified laboratory spike and one laboratory blank were analyzed with each set of 10 to 16 field samples. Laboratory reagent blanks were used to assess potential sample contamination. Laboratory recoveries for four surrogate standards analyzed in reagent-water samples fortified at $0.5 \mu \mathrm{g} / \mathrm{L}$ at the USGS-NWQL during method development averaged 72 percent plus or minus 8 percent relative standard deviation (Zaugg and others, 2006). The average recoveries for the surrogate standards of bisphenol A-d3, caffeine-13C, decafluorobiphenyl, and fluoranthene-d10 that were analyzed concurrently with study samples were $67,82,72$, and 83 percent, respectively. Surrogate recoveries were low (less than 50 percent) for a few environmental samples (appendix 2): the downstream-1 site on the South Fork Crow River (SFCROWDS1) on May 9, 2007; the upstream site on the Redwood River (REDWD-US1) on May 10, 2007; and the downstream-1 site on the Redwood River (REDWD-DS1) on August 28, 2007.

The concentrations of 20 chemicals for analyses at the USGS-NWQL using schedule 4433 (coded with an "E" in table 4) are reported as estimated for one of three reasons: unacceptably low-biased recovery (less than 60 percent) or highly variable method performance (greater than 25 percent relative standard deviation), unstable instrument response, or reference standards prepared from technical mixtures. Nine additional chemicals (coded with an "e" in table 4) had variable performance during the initial method validation. The concentration of these chemicals is reported as estimated if the spike recovery or expected continuing calibration verification concentrations for each set of samples were not within control limits (Zaugg and others, 2006).

Field quality-assurance samples were used to assess collection and processing techniques. Field quality-assurance samples included blanks and replicates. Potential contamination of water samples during collection and sample processing was assessed with field-blank samples. Field blanks were prepared at the sampling sites before the collection of the corresponding environmental sample. Blank samples were prepared by processing high-performance liquid-chromatography-grade organic-free water (Baker Analyzed, J.T. Baker Co.) through the same equipment used to collect and process field samples. Six sets of field blank samples were collected and analyzed to assess contamination introduced during sample collection and processing for water samples. Four blank samples were analyzed for chloride and sulfate, and one sample was analyzed for nutrients and wastewater indicator chemicals at the USGS-NWQL by using schedule 4433. All six blank samples were analyzed for organic carbon, EDTA/NTA/NPECs, and wastewater indicator chemicals at the USGS-NRP.

Concentrations of chemicals in the environmental samples that were nearly the same as concentrations found in the blank samples may be the result of field or laboratory contamination. Eight chemicals were detected among the six blank samples analyzed (table 7). Ammonia was detected in one blank sample at an estimated concentration of $0.015 \mathrm{mg} / \mathrm{L}$ (appendix 3), which was less than the MRL. More than 90 percent of the environmental samples had ammonia concentrations greater than $0.015 \mathrm{mg} / \mathrm{L}$ (table 7). However, ammonia detections in two samples that were collected from the North Branch of the Grindstone River (GRIND-US1) and from the Grindstone River below Hinckley (GRIND-DS1) on August 22, 2007, had concentrations that were estimated below the MRL and in the same concentration range as the 
field blank. Less than 50 percent of the environmental samples had concentrations of 2,6-di-tert-butyl-4-methylphenol and cholesterol that were greater than concentrations measured in the field blank samples (table 7). The chemicals 2,6-di-tertbutyl-4-methylphenol and OP2EO were detected in only one field blank sample, whereas cholesterol was detected in four blank samples (appendix 1).

Field replicate samples were used to determine variability of detection and concentration that result from sample processing techniques (sample splitting, filtration, and transport). Replicate samples consist of a split of the environmental sample, so the environmental and replicate samples should be nearly equal in composition. Four replicate pairs were analyzed for chloride and sulfate at the USGS-NWQL (appendix 4). One replicate and environmental sample pair was analyzed for nutrients at the NWQL (appendix 3). Five replicate pairs were analyzed for organic carbon at the USGS-NRP (appendix 1). Four replicate pairs were analyzed for EDTA, NTA, and NP1EC-NP4EC at the USGS-NRP (appendix 1). Five replicate pairs were analyzed for wastewater indicator chemicals at the USGS-NRP (appendix 1). Two replicate pairs were analyzed for laboratory schedule 4433 at the USGS-NWQL (appendix 2).

The variability between environmental and field replicate sample pairs was assessed with a comparison of a calculation of the relative percent difference $(R P D)$ between the two samples as follows:

$$
R P D=\frac{(E N V-R E P)}{\text { Average }(E N V+R P D)} \times 100
$$

where $E N V$ is the concentration the environmental sample, and $R E P$ is the concentration in the replicate sample. In addition, the detection consistency (percentage of times a chemical was consistently detected or not detected in the replicate and the environmental sample) was calculated.

The average RPD for the replicate and corresponding environmental samples for all major ions and nutrients was 1 percent (table 8). Average RPDs for the four pairs were 0.3 percent for chloride and 1 percent for sulfate. The RPDs for individual nutrients were all less than 4 percent. Average RPDs for TOC, $\mathrm{UV}_{254}$, and $\mathrm{SUVA}_{254}$ analyzed at the USGSNRP also were low, ranging from 9 to 13 percent.

Twenty-eight chemicals (excluding TOC, $\mathrm{UV}_{254}$, and $\mathrm{SUVA}_{254}$ ) were detected among all replicate and environmental samples analyzed at the USGS-NRP (table 8; appendix 1). Twenty-one chemicals were detected in both the replicate and corresponding environmental samples among all pairs. RPDs for those 21 chemicals ranged from 0 to 193 percent; the average was 31 percent. The average detection consistency was 88 percent among all chemicals analyzed.

Four replicate pairs were analyzed for EDTA, NTA, and NPECs. EDTA, NTA, NP1EC, and 4-nonylphenoldiethoxycarboxylate (NP2EC) were detected in at least one of the four replicate sample pairs analyzed at the USGS-NRP; the aver-

Table 7. Summary of chemicals detected in field blank samples.

[MRL, minimum reporting level; NWQL, National Water Quality Laboratory; USGS-NRP, U.S. Geological Survey National Research Program Laboratory; $\mathrm{mg} / \mathrm{L}$, milligrams per liter; $\mathrm{ng} / \mathrm{L}$, nanograms per liter; E, estimated; only chemicals with at least one detection are listed. Concentration ranges are for detected chemicals]

\begin{tabular}{|c|c|c|c|c|c|c|}
\hline $\begin{array}{l}\text { Chemical name } \\
\text { (unit of measure) }\end{array}$ & MRL & $\begin{array}{l}\text { Number } \\
\text { of blank } \\
\text { samples } \\
\text { analyzed }\end{array}$ & $\begin{array}{c}\text { Number } \\
\text { of } \\
\text { detections }\end{array}$ & $\begin{array}{l}\text { Concentration } \\
\text { range } \\
\text { in } \\
\text { blank } \\
\text { samples }\end{array}$ & $\begin{array}{l}\text { Concentration } \\
\text { range } \\
\text { in } \\
\text { environmental } \\
\text { samples }\end{array}$ & $\begin{array}{c}\text { Percent of } \\
\text { environmental } \\
\text { samples with } \\
\text { concentrations } \\
\text { greater than } \\
\text { blank-sample } \\
\text { concentrations }\end{array}$ \\
\hline \multicolumn{7}{|c|}{ NWQL major lons and nutrients (mg/L) } \\
\hline Sulfate & 0.18 & 1 & 1 & E0.114 & $0.91-1,380$ & 100 \\
\hline Nitrogen, ammonia as nitrogen & .02 & 1 & 1 & E0.015 & $0.013-3.99$ & 94 \\
\hline \multicolumn{7}{|c|}{ USGS-NRP wastewater indicators (ng/L) } \\
\hline 2,6-Di-tert-butyl-1,4-benzoquinone & 5 & 6 & 1 & 110.0 & $6.10-800.2$ & 65 \\
\hline 2,6-Di-tert-butyl-4-methylphenol & 5 & 6 & 1 & 13.2 & $5.14-210$ & 28 \\
\hline 3-beta-Coprostanol & 5 & 6 & 1 & 25.8 & $17.00-3,600$ & 95 \\
\hline $\begin{array}{l}\text { 4-Nonylphenolmonoethoxylate } \\
\text { (NP1EO) }\end{array}$ & 50 & 6 & 2 & $79.7-82.1$ & $58.0-660.5$ & 68 \\
\hline $\begin{array}{l}\text { 4-tert-Octylphenoldiethoxylate } \\
\text { (OP2EO) }\end{array}$ & 5 & 6 & 1 & 9.9 & $10.7-130.0$ & 100 \\
\hline Cholesterol & 5 & 6 & 4 & $77.8-330.0$ & $10.7-2,200.0$ & 44 \\
\hline
\end{tabular}


Table 8. Concentrations and detections of chemicals for which environmental and replicate samples were analyzed.

[ENV, environmental sample; REP, replicate sample; RPD, relative percent difference; USGS-NRP, U.S. Geological Survey National Research Program Laboratory; NWQL, National Water Quality Laboratory; mg/L, milligrams per liter; $\mu \mathrm{g} / \mathrm{L}$, micrograms per liter; ng/L, nanograms per liter; --, not determined]

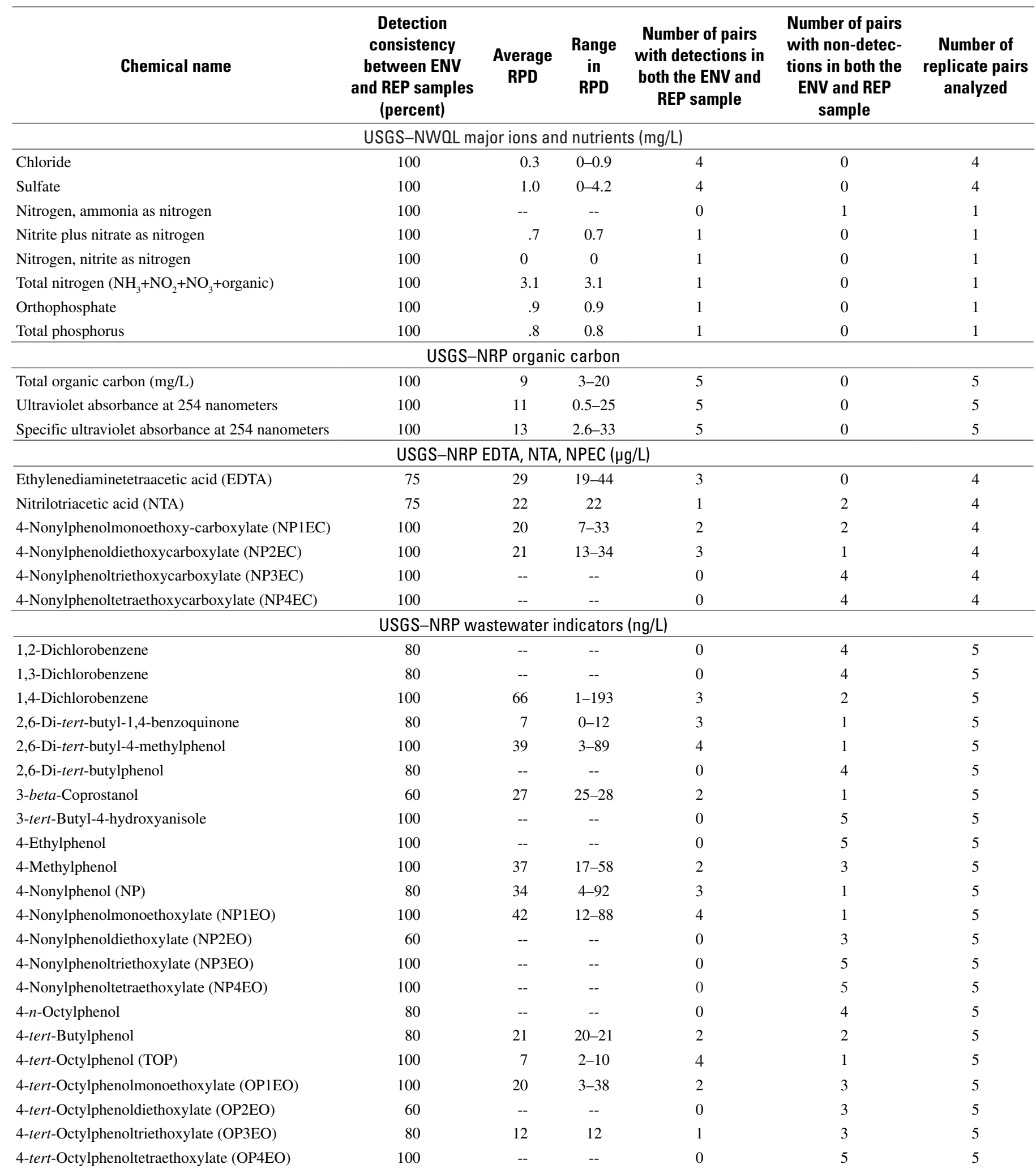


Table 8. Concentrations and detections of chemicals for which environmental and replicate samples were analyzed.—Continued

[ENV, environmental sample; REP, replicate sample; RPD, relative percent difference; USGS-NRP, U.S. Geological Survey National Research Program Laboratory; NWQL, National Water Quality Laboratory; mg/L, milligrams per liter; $\mu \mathrm{g} / \mathrm{L}$, micrograms per liter; ng/L, nanograms per liter; --, not determined]

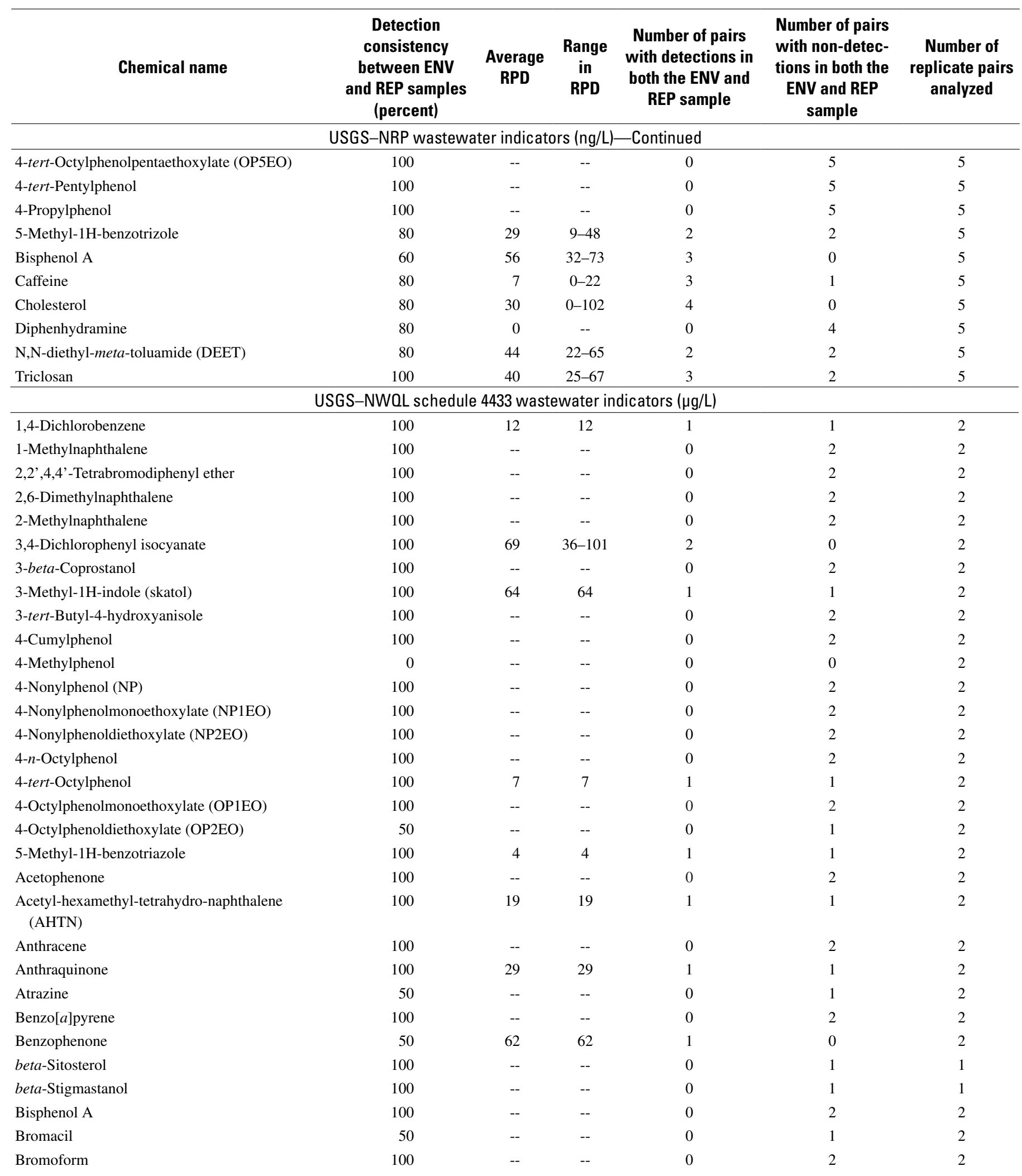


Table 8. Concentrations and detections of chemicals for which environmental and replicate samples were analyzed.—Continued

[ENV, environmental sample; REP, replicate sample; RPD, relative percent difference; USGS-NRP, U.S. Geological Survey National Research Program Laboratory; NWQL, National Water Quality Laboratory; mg/L, milligrams per liter; $\mu \mathrm{g} / \mathrm{L}$, micrograms per liter; ng/L, nanograms per liter; --, not determined]

\begin{tabular}{|c|c|c|c|c|c|c|}
\hline Chemical name & $\begin{array}{c}\text { Detection } \\
\text { consistency } \\
\text { between ENV } \\
\text { and REP samples } \\
\text { (percent) }\end{array}$ & $\begin{array}{c}\text { Average } \\
\text { RPD }\end{array}$ & $\begin{array}{c}\text { Range } \\
\text { in } \\
\text { RPD }\end{array}$ & $\begin{array}{l}\text { Number of pairs } \\
\text { with detections in } \\
\text { both the ENV and } \\
\text { REP sample }\end{array}$ & $\begin{array}{l}\text { Number of pairs } \\
\text { with non-detec- } \\
\text { tions in both the } \\
\text { ENV and REP } \\
\text { sample }\end{array}$ & $\begin{array}{c}\text { Number of } \\
\text { replicate pairs } \\
\text { analyzed }\end{array}$ \\
\hline \multicolumn{7}{|c|}{ USGS-NWOL schedule 4433 wastewater indicators $(\mu \mathrm{g} / \mathrm{L})$-Continued } \\
\hline Caffeine & 100 & 19 & 19 & 1 & 1 & 2 \\
\hline Camphor & 100 & -- & -- & 0 & 2 & 2 \\
\hline Carbaryl & 100 & -- & -- & 0 & 2 & 2 \\
\hline Carbazole & 100 & -- & -- & 0 & 2 & 2 \\
\hline Chlorpyrifos & 100 & -- & -- & 0 & 2 & 2 \\
\hline Cholesterol & 50 & 8 & 8 & 1 & 0 & 2 \\
\hline Cotinine & 100 & -- & -- & 0 & 2 & 2 \\
\hline Diazinon & 100 & -- & -- & 0 & 2 & 2 \\
\hline Dichlorvos & 100 & -- & -- & 0 & 2 & 2 \\
\hline Diethyl phthalate & 100 & -- & -- & 0 & 2 & 2 \\
\hline Diethylhexyl phthalate & 50 & -- & -- & 0 & 1 & 2 \\
\hline$d$-Limonene & 100 & -- & -- & 0 & 2 & 2 \\
\hline Fluoranthene & 100 & 4 & 4 & 1 & 1 & 2 \\
\hline $\begin{array}{l}\text { Hexahydrohexamethyl-cyclopenta-benzopyran } \\
\text { (HHCB) }\end{array}$ & 50 & 6 & 6 & 1 & 0 & 2 \\
\hline Indole & 100 & -- & -- & 0 & 2 & 2 \\
\hline Isoborneol & 100 & -- & -- & 0 & 2 & 2 \\
\hline Isophorone & 0 & -- & -- & 0 & 0 & 2 \\
\hline Isopropylbenzene (cumene) & 100 & -- & -- & 0 & 2 & 2 \\
\hline Isoquinoline & 100 & -- & -- & 0 & 2 & 2 \\
\hline Menthol & 100 & -- & -- & 0 & 2 & 2 \\
\hline Metalaxyl & 100 & -- & -- & 0 & 2 & 2 \\
\hline Methyl salicylate & 100 & -- & -- & 0 & 2 & 2 \\
\hline Metolachlor & 100 & 8 & 8 & 1 & 1 & 2 \\
\hline N,N-diethyl-meta-toluamide (DEET) & 100 & 9 & $8-10$ & 2 & 0 & 2 \\
\hline Naphthalene & 100 & -- & -- & 0 & 2 & 2 \\
\hline Pentachlorophenol & 100 & -- & -- & 0 & 2 & 2 \\
\hline Phenanthrene & 100 & -- & -- & 0 & 2 & 2 \\
\hline Phenol & 100 & -- & -- & 0 & 2 & 2 \\
\hline Prometon & 100 & 17 & 17 & 1 & 1 & 2 \\
\hline Pyrene & 100 & 13 & 13 & 1 & 1 & 2 \\
\hline Tetrachloroethene & 50 & -- & -- & 0 & 1 & 2 \\
\hline Tri(2-butoxyethyl) phosphate & 100 & 28 & $24-33$ & 2 & 0 & 2 \\
\hline Tri(2-chloroethyl) phosphate & 100 & 19 & $16-23$ & 2 & 0 & 2 \\
\hline Tri(dichloroisopropyl) phosphate & 50 & 20 & 20 & 1 & 0 & 2 \\
\hline Tributyl phosphate & 100 & -- & -- & 0 & 2 & 2 \\
\hline Triclosan & 50 & -- & -- & 0 & 1 & 2 \\
\hline Triethyl citrate (ethyl citrate) & 100 & 3 & 3 & 1 & 1 & 2 \\
\hline Triphenyl phosphate & 100 & 28 & $14-42$ & 2 & 0 & 2 \\
\hline
\end{tabular}


age RPD was 24 percent. The average detection consistency was 92 percent for these chemicals.

Five replicate pairs were analyzed for wastewater indicator chemicals at the USGS-NRP. The average RPD for 17 wastewater indicator chemicals detected among five environmental and corresponding replicate samples analyzed at the USGS-NRP was 31 percent. The average detection consistency was 32 percent for these chemicals.

Twenty-nine chemicals were detected among the two replicate and corresponding environmental sample pairs analyzed by using USGS-NWQL schedule 4433 (table 8, appendix 2). RPDs for those 29 chemicals ranged from 3 to 101 percent; the average RPD was 23 percent. The average detection consistency was 88 percent among all chemicals analyzed.

Two laboratory-set blanks and one replicate sample were analyzed at the USGS-NRP for quality assurance of bed-sediment samples (appendix 5). The chemicals 2,6-di-tert-butyl1,4-benzoquinone, TOP, and triclosan were detected in one laboratory-set blank sample during bed-sediment analyses. TOP and tricolsan were not detected in environmental samples. The chemical 2,6-di-tert-butyl-1,4-benzoquinone was detected in most environmental samples at concentrations ranging from 9.3 to $66 \mathrm{ng} / \mathrm{L}$, which were similar to the concentration found in the blank sample $(12 \mathrm{ng} / \mathrm{L})$. One field bedsediment replicate sample was collected at the South Branch Grindstone River (site GRINDD-US2) and analyzed for the chemicals listed in table 5. Five chemicals were detected in the environmental and replicate samples. Four of these chemicals were detected in both the environmental and corresponding replicate samples. The RPDs between the replicate and environmental samples ranged from 9 to 78 percent (average of 26 percent).

A rigorous quality-assurance protocol was followed at the St. Cloud State University Aquatic Toxicology Laboratory to ensure the accuracy of Vtg analyses and histopathology characterizations. All plasma samples were analyzed in triplicate to determine variation in test results. Coefficients of variation (standard deviation divided by the mean) were calculated to assess variation in analytical results for the Vtg data. The coefficients of variation were within 10 percent, which is the St. Cloud State University standard for variation. Vtg was detected in selected samples below the detection limit of $0.54 \mu \mathrm{g} / \mathrm{mL}$. The concentrations for these samples are estimated because the values are outside the acceptable quantification limits. All histopathology characterizations followed U.S. Environmental Protection Agency (2006b) protocols and was performed by one trained laboratory member. In the field, fish were kept alive until dissection to preserve organ and Vtg integrity. All dissections were performed by the same field crew of three people. Multiple sections of testes and livers were taken from each male fish in order to obtain a representative sample of these organs for histological characterization. In the laboratory, three slides of the testes and livers were made and blindly assessed according to U.S. Environmental Protection Agency (2006b) protocol.

\section{Data Description}

A description of the hydrologic characteristics, water and bed-sediment chemistry, WWTP monitoring data, and fish characteristics measured or collected during this study follow in this section. Hydrologic data are provided to set the context for the chemical and biological data. Links to Microsoft Excel files containing data (appendixes 1-10) are provided within each section.

\section{Hydrologic Characteristics}

Streamflow was greater in the Redwood and South Fork Crow Rivers than in the Grindstone River (figs. 13, 14, and 15; appendix 6). Streamflow was least during winter under ice cover and greatest March and April during snowmelt runoff. Before and during the first sample collection in February 2007, solid ice covered all streams. The remainder of sampling events occurred during snowmelt runoff, the recession of the snowmelt runoff, in early summer after precipitation events, and during summer and fall low-flow periods.

Instantaneous streamflow measurements made at the time of sampling and streamflow from USGS streamflow gaging stations at or near the sampling locations on each river were used to characterize hydrologic conditions during sampling (figs. 13-15). Two nearby USGS gaging stations were used to characterize the hydrologic conditions at the sampling locations on each stream. USGS gaging stations located on the Middle Fork of the Crow River near Spicer (05278000) and downstream from the sampling reach at the Crow River at Rockford, Minn. (05280000) were used to characterize the flow conditions for the South Fork Crow River. USGS gaging stations located at the upstream site on the Redwood River near Marshall (05315000), and at a downstream site at the Redwood River near Redwood Falls (05316500) were used to characterize the Redwood River. USGS gaging stations located on Stoney Brook near Brookston (04021520) and a station downstream from the sampling reach at the Kettle River below Sandstone (05336700) were used to characterize the Grindstone River.

The percentage of streamflow contributed by WWTP effluent was determined for three locations on each stream: the stream at the WWTP discharge location, the first site downstream from the WWTP, and the second site downstream from the WWTP. The percentage of streamflow contributed by WWTP effluent was calculated by dividing the discharge from the WWTP by streamflow. The percentage of streamflow contributed by WWTP effluent ranged from less than 1 to 79 percent among all sites and all sampling periods (appendix 6). In general, the percentage of flow composed of WWTP effluent was greatest in the South Fork Crow River and least in the Grindstone River. The greatest percentage of streamflow was contributed by WWTP effluent in the South Fork Crow and Redwood Rivers during February 2007, when streamflow was low (figs. 13 and 14). The lowest percent- 

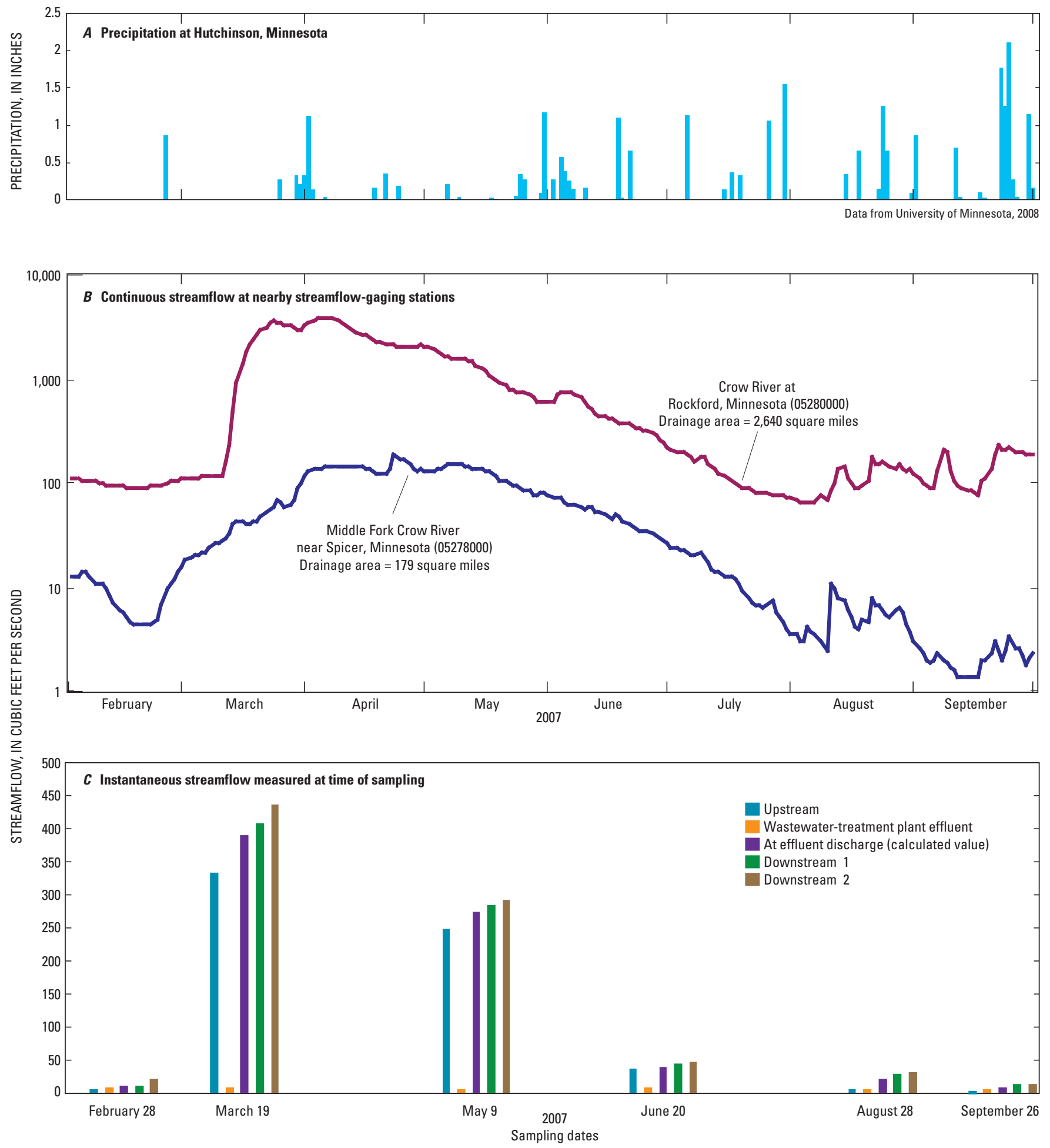

Figure 13. Hydrologic characteristics of $A$, precipitation, $B$, continuous streamflow at nearby streamflow gaging stations, and $C$, instantaneous streamflow measured at the time of sampling for the South Fork Crow River. 

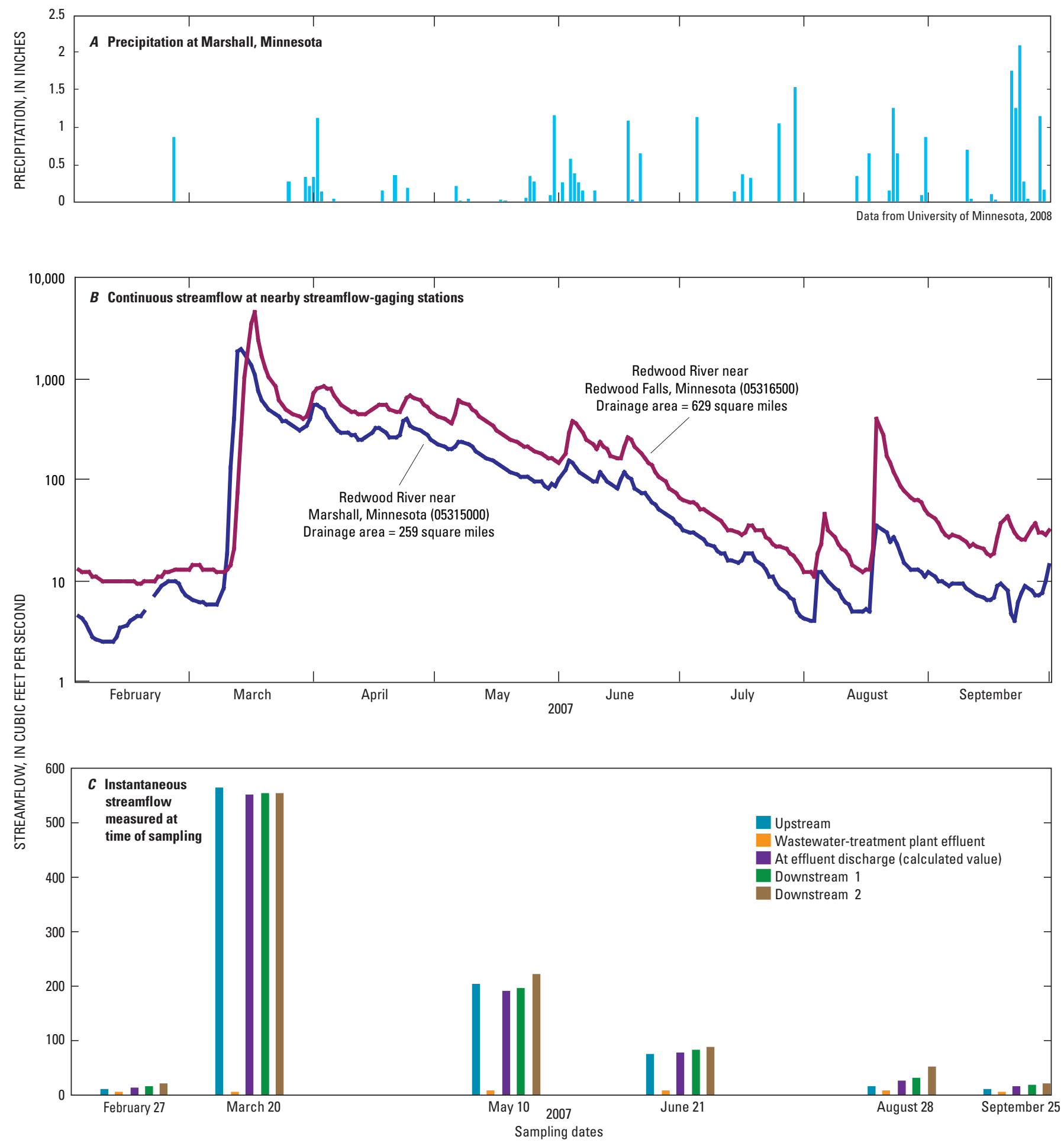

Figure 14. Hydrologic characteristics of $A$, precipitation, $B$, continuous streamflow at nearby streamflow gaging stations, and $C$, instantaneous streamflow measured at the time of sampling for the Redwood River. 

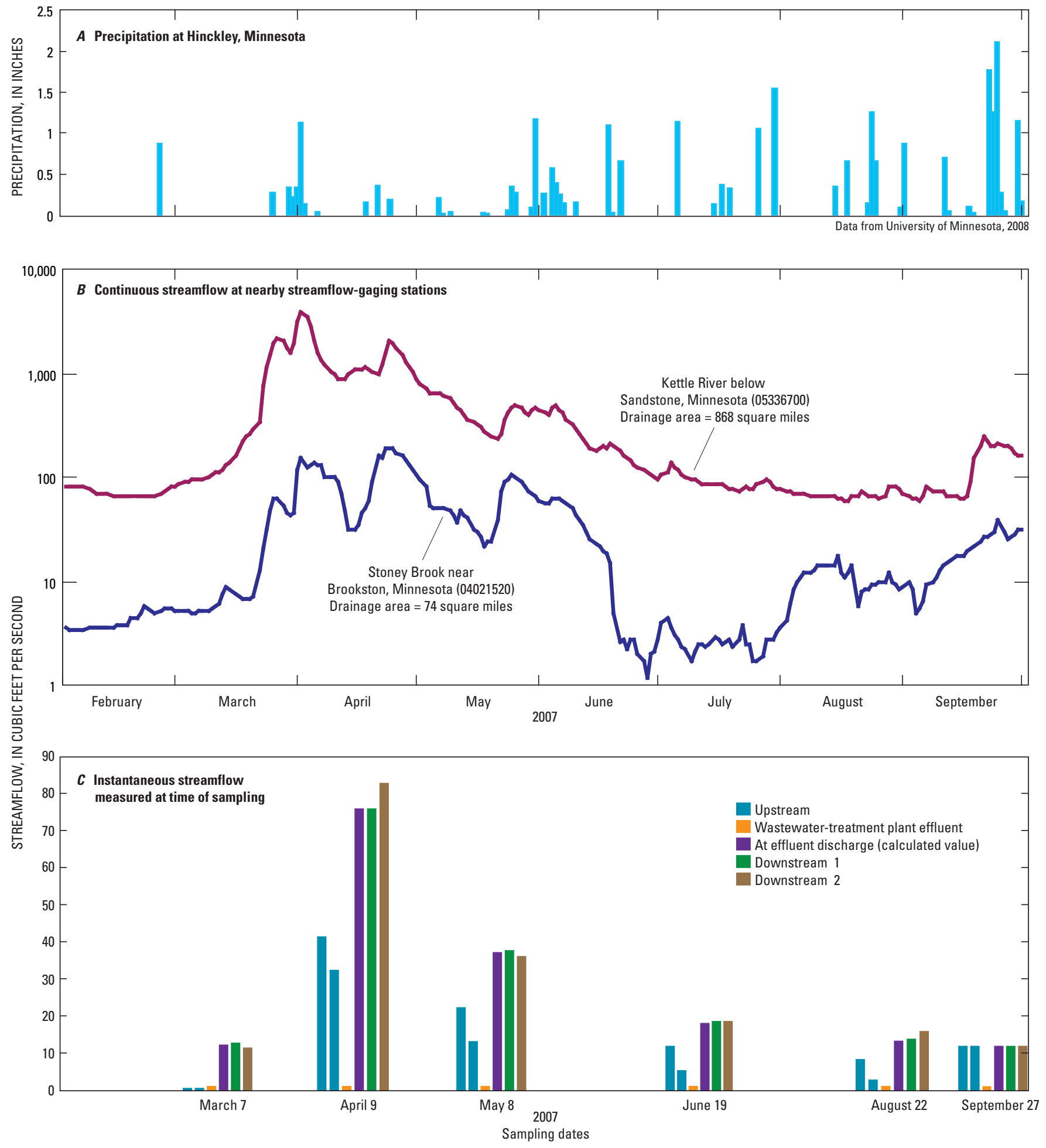

Figure 15. Hydrologic characteristics of $A$, precipitation, $B$, continuous streamflow at nearby streamflow gaging stations, and $C$, instantaneous streamflow measured at the time of sampling for the Grindstone River. 
ages of effluent on those two streams occurred during March and May when streamflows were at or near their peak. As the summer progressed, streamflows receded and the percentage of flow contributed by WWTP effluent gradually increased. By September, the percentage of effluent was nearly equal to the percentage of effluent during winter under-ice sampling. In contrast to the other two streams, WWTP effluent in the Grindstone River contributed the greatest percentage of flow during August as streamflows were receding and the amount of WWTP discharge increased. The percentage of flow contributed by WWTP effluent was less in the Grindstone River in September than in other streams owing to an increase in streamflow before sampling (fig. 15).

\section{Water Chemistry}

Physical measurements of dissolved oxygen, $\mathrm{pH}$, specific conductance, and water temperature provide information about the sampled streams that may influence the fate of the selected chemicals and those field measurements are listed in appendix 7. Dissolved oxygen concentrations ranged from $1.6 \mathrm{mg} / \mathrm{L}$ to $15.4 \mathrm{mg} / \mathrm{L}$ among stream samples and from 6.6 to $11.3 \mathrm{mg} / \mathrm{L}$ among WWTP effluent samples. Dissolved oxygen concentrations at the South Fork Crow River were lower at the upstream site than at the downstream sites during all sampling periods. This pattern also was observed at the Redwood River sites during most sampling periods.

Specific conductance values ranged from 78 to 2,500 microsiemens per centimeter at $25^{\circ} \mathrm{C}(\mu \mathrm{S} / \mathrm{cm})$ for stream samples and from 1,350 to 5,060 $\mu \mathrm{S} / \mathrm{cm}$ in WWTP effluent samples. Average specific conductance in stream samples was greatest at the Redwood River $(1,260 \mu \mathrm{S} / \mathrm{cm})$, followed by the South Fork Crow River $(799 \mu \mathrm{S} / \mathrm{cm})$ and the Grindstone River $(189 \mu \mathrm{S} / \mathrm{cm})$. Specific conductance values generally were greater at stream sites downstream from WWTP effluent than at the upstream sites, and the specific conductance values were much greater in WWTP effluent than in the streams.

Stream temperatures at upstream sites, measured when samples were collected, ranged from $32^{\circ} \mathrm{F}$ to $77^{\circ} \mathrm{F}$ among all streams and sampling periods. Temperatures in WWTP effluent ranged from 44 to $76^{\circ} \mathrm{F}$. Temperatures varied seasonally in streams. The coolest measured stream temperatures were in February and March, and the warmest measured stream temperatures were in June. WWTP effluent temperatures were greater than stream temperatures in the winter, spring, and fall and lower than stream temperatures during June.

Calcium, magnesium, potassium, sodium, and chloride concentrations were greater at upstream sites on the South Fork Crow and Redwood Rivers than upstream sites on the Grindstone River (appendix 4). Dissolved iron concentrations were greatest at the Grindstone River sites, whereas dissolved sulfate concentrations were greatest at the Redwood River sites. Most major-ion concentrations were lower in stream samples than WWTP effluent samples.
Total nitrogen and total phosphorus concentrations were greatest at the South Fork Crow River followed by the Redwood River, and then the Grindstone River (appendix 3). Total nitrogen (nitrate + nitrite + ammonia + organic $\mathrm{N}$ ) and total phosphorus concentrations were greater at sites downstream from WWTP effluent discharges than at the upstream sites along on each river.

TOC concentrations (appendix 1) ranged from 3.9 to $22.7 \mathrm{mg} / \mathrm{L}$ among environmental samples. Average TOC concentrations in stream samples were greatest in the South Fork Crow River $(12.4 \mathrm{mg} / \mathrm{L})$ followed by the Grindstone River $(11.8 \mathrm{mg} / \mathrm{L})$ and the Redwood River $(8.5 \mathrm{mg} / \mathrm{L})$. TOC concentrations in WWTP effluent ranged from 5.8 to $11.9 \mathrm{mg} / \mathrm{L}$ among all WWTPs. The Hinckley effluent (site HINCKWWTP) tended to have the lowest TOC concentrations among effluent samples. SUVA ${ }_{254}$ ranged from 1.5 to 4.6 among stream samples and was greatest in samples collected from the Grindstone River compared to other streams. SUVA $_{254}$ was lower in WWTP effluent than in stream samples from the Redwood and Grindstone Rivers.

Thirty five of the 38 chemicals (excluding TOC, $\mathrm{UV}_{254}, \mathrm{SUVA}_{254}$ ) analyzed at the USGS-NRP (table 4) were detected among all samples (appendix 1). The chemicals detected most frequently (in greater than 40 percent of the environmental samples) were 2,6-di-tert-butyl-1,4-benzoquinone, 2,6-di-tert-butyl-4-methylphenol, 3-beta-coprostanol, 4-methylphenol, NP, TOP, bisphenol A, cholesterol, EDTA, and triclosan.

EDTA concentrations ranged from 0.7 to $960 \mu \mathrm{g} / \mathrm{L}$ among all environmental samples (appendix 1). EDTA concentrations were greater in WWTP effluent samples than in stream samples. EDTA was detected in a few samples from sites upstream from WWTP effluent discharge at concentrations of $11 \mu \mathrm{g} / \mathrm{L}$ or less. EDTA was detected at sites downstream from WWTP effluent discharge in each stream at concentrations ranging from 0.7 to $62 \mu \mathrm{g} / \mathrm{L}$.

NP1EC, NP2EC, and 4-nonylphenoltriethoxycarboxylate (NP3EC) were detected in study samples, and NP1EC and NP2EC were the dominant NPECs detected (appendix 1). Excluding nondetections, the sum of NP1EC through NP3EC concentrations ranged from 5.1 to $260 \mu \mathrm{g} / \mathrm{L}$ among all samples. NPECs were detected in effluent samples from all three WWTPs. NPECs were detected at downstream sites on the Redwood River (sites REDWD-DS1 and REDWD-DS2) and on the South Fork Crow River (site SFCROW-DS1); NPECs were not detected at the downstream sites on the Grindstone River.

Alkylphenols and alkylphenol ethoxylates were detected in upstream, effluent, and downstream samples in each stream (appendix 1). NP was detected in 49 percent of environmental samples. Excluding nondetections, concentrations of NP ranged from 100 to $880 \mathrm{ng} / \mathrm{L}(0.10$ to $0.88 \mu \mathrm{g} / \mathrm{L})$ among all samples. The single greatest NP concentration in a stream sample was measured at the second downstream site on the Grindstone River. TOP concentrations in effluent samples were greatest at the Hinckley WWTP, where the maximum 
TOP concentration of $110 \mathrm{ng} / \mathrm{L}$ was measured. NP1EO and NP2EO were detected at upstream sites, effluent sites, and downstream sites along each stream. NP1EO was detected more commonly than NP2EO. NPEOs were detected more commonly at the Redwood River and Grindstone River sites than at the South Fork Crow River sites. Concentrations and frequency of detection of NP1EO and NP2EO in effluent samples were greater at Marshall and Hinckley than at Hutchinson (appendix 1).

Bisphenol A and triclosan were detected in upstream, effluent, and downstream samples in all three basins (appendix 1). Bisphenol A concentrations ranged from 4.87 to $137.40 \mathrm{ng} / \mathrm{L}$ among all samples. Triclosan concentrations ranged from 7.04 to $150 \mathrm{ng} / \mathrm{L}$ among all samples. Two additional wastewater indicator compounds (caffeine and 1,4dichlorobenzene) were commonly detected in effluent samples and in samples from sites downstream from effluent discharge; there were very few detections of these compounds in samples from upstream sites.

Thirty-seven organic chemicals were detected among all environmental samples (26 samples) by using USGS-NWQL analytical schedule 4433 (69 chemicals analyzed, excluding surrogates) (appendix 2). The chemicals 3,4-dichlorophenyl isocyanate, AHTN, benzophenone, cholesterol, DEET, HHCB, and tri(dichloroisopropyl) phosphate were the most commonly detected (in at least 30 percent of the samples) chemicals among all samples.

More chemicals analyzed by using NWQL analytical schedule 4433 were detected in WWTP effluent samples than in stream samples for most sites. The average number of chemicals detected was 2 per upstream sample, 16 per WWTP sample, 6 per downstream-1 sample, and 6 per downstream- 2 sample. More chemicals were detected during the fall than during the spring sampling period at the South Fork Crow River and Redwood River. Fewer chemicals were detected in the Grindstone River than in the other two streams.

The chemicals TOP and OP2EO were the only two APs or APEOs detected by using NWQL schedule 4433 (appendix 2). Some chemicals were detected in all effluent samples (1,4-dichlorobenzene, benzophenone, HHCB, DEET, triethyl citrate, tri(2-chloroethyl) phosphate, and tri(dichloroisopropyl) phosphate), and others were detected in most (five out of six) WWTP effluent samples (3-methyl-1H-indole, 4-methylphenol, and AHTN). Most of these chemicals also were detected in corresponding samples at downstream sites with the exception of 3-methyl-1H-indole, 4-methylphenol, 1,4-dichlorobenzene, and triethyl citrate, which were not detected in any downstream samples. The chemicals, AHTN, anthraquinone, atrazine, beta-sitosterol, cholesterol, diethylhexyl phthalate, fluoranthene, HHCB, isophorone, metolachlor, DEET, phenanthrene, and pyrene were detected at sites upstream from WWTP effluent discharge.

As part of the time-series data collection, atrazine was detected in 60 of the 62 samples collected, and concentrations ranged from 1.93 to $46.41 \mathrm{ng} / \mathrm{L}$ among all samples (appendix 8). Atrazine concentrations were lower and stable from April through the end of May, increased after June 1, and then began to decline at the end of June. Total APs were detected in 5 of the 62 samples collected at concentrations ranging from 50 to $65 \mathrm{ng} / \mathrm{L}$. The five AP detections occurred from May 10 through June 16, 2007.

\section{Wastewater-Treatment Plant Monitoring Data}

WWTPs monitor selected properties and constituents including CBOD5, percent removal of CBOD5, ammonia, total phosphorus, and total suspended solids as part of annual reporting requirements of the Minnesota Pollution Control Agency. These properties and constituents indicate effluent characteristics and treatment operations. Selected properties and constituents that were monitored at the three WWTPs (Marshall, Hutchinson, and Hinckley), upstream WWTPs, and at one major industrial discharger (discharge greater than $1 \mathrm{Mgal} / \mathrm{d}$ ) are listed in appendix 9; data presented are from Minnesota Pollution Control Agency, written commun., 2008.

The maximum weekly average CBOD5 varied during the sampling period (January-September 2007) among sites and within a site. For the three WWTPs studied, the maximum weekly average CBOD5 ranged from 2 to $8 \mathrm{mg} / \mathrm{L}$ among the sampling periods.

Monthly average nitrogen ammonia concentrations varied among WWTPs and sampling periods; concentrations ranged from less than 1 to $8 \mathrm{mg} / \mathrm{L}$ and were greatest in the Hinckley effluent and least in the Marshall effluent. Nitrogen ammonia concentrations in the Hinckley WWTP effluent were greater during May, June, and July than at other times sampled.

In effluent samples from the three selected WWTPs, total suspended solids concentrations ranged from 3 to $18 \mathrm{mg} / \mathrm{L}$ and generally were least in the Marshall WWTP effluent. Total phosphorus concentrations ranged from less than 1 to $8.8 \mathrm{mg} / \mathrm{L}$ and were greatest in the Marshall effluent, followed by the Hutchinson and Hinckley effluents.

\section{Bed-Sediment Chemistry}

Fewer chemicals were detected in bed-sediment samples than in water samples analyzed during the same sampling period. Nine of 29 chemicals analyzed for were detected in bed-sediment samples (appendix 5). The chemicals 2,6-di-tertbutyl-1,4-benzoquinone, 3-beta-coprostanol, 4-methylphenol, and cholesterol were detected in more than 90 percent of the bed-sediment samples. NP and NP1EO were detected only at sites downstream from WWTP discharge locations. NP was detected at downstream locations in all three streams at concentrations ranging from 24-260 nanograms per gram (ng/g), and NP1EO was detected at the two downstream locations on the Grindstone River at concentrations of 15 and $17 \mathrm{ng} / \mathrm{g}$. Excluding one nondetection, concentrations of 3-betacoprostanol ranged from 39 to $240 \mathrm{ng} / \mathrm{g}$ among all samples, and the average concentration was greater in bed-sediment 
samples from the Grindstone River than in bed-sediment samples from the other two streams. Cholesterol concentrations ranged from 220 to $820 \mathrm{ng} / \mathrm{g}$ among all bed-sediment samples and were greater at the Grindstone River than at other streams.

\section{Fish Characteristics}

During this study, 508 male and 397 female fish were collected (appendix 10). Weights and lengths were measured and condition factors were calculated for all fish. Complete plasma and histological analysis was attempted for all male fish collected, because male fish were the principal focus of the evaluation of biological responses to EACs. Among all sites, 56 male common carp, 152 male common shiners, 46 male creek chubs, 127 male fathead minnows, 74 male redhorse, and 53 male white suckers were collected. It was not possible to perform all analyses on all fish owing to fish size or reproductive stage, and availability of tissue or plasma for characterization. For example, gonads of male fish weighing less than 7 ounces (200 g) were not weighed because the analytical equipment for field applications was not accurate enough; this weight limit precluded GSI analyses for fish less than 7 ounces. Histological characterizations were not possible on male fish that were either too immature or in an advanced reproductive stage. Also it was not always possible to obtain a plasma sample that was sufficient for Vtg analyses.

Condition factors in all male fish ranged from 0.12 to 2.35; the average condition factor was 1.12 (appendix 10). When all species were combined, male fish at the South Fork Crow River had the greatest average condition factor (1.20, number sampled $(n)=199)$ compared with average conditions factors of 1.07 and 1.05 at the Grindstone and Redwood Rivers, respectively. The condition factors were similar at upstream and downstream sites (1.10 and 1.12, respectively) with all fish and sites combined.

Vtg analysis (minimum detection limit of $0.54 \mu \mathrm{g} / \mathrm{mL}$ ) was performed on 415 of the 508 male fish (histologically determined) from which sufficient blood was drawn. Of those fish, 112 male fish were from the Grindstone River, 157 were from the South Fork Crow River, and 146 were from the Redwood River. Vtg was detected in 57 percent of the male fish sampled; concentrations ranged from an estimated value of 0.1 to $330 \mu \mathrm{g} / \mathrm{mL}$ (average of $17.1 \mu \mathrm{g} / \mathrm{mL}$ ) for the 237 male fish with detections. Vtg analyses also were done on 137 female fish, in which Vtg was detected in 71 percent; concentrations ranged from 0.059 to $54,300 \mu \mathrm{g} / \mathrm{mL}$ (average of $1,538.4 \mu \mathrm{g} / \mathrm{mL}$ ). Male fathead minnows had the greatest average Vtg concentration $(102.50 \mu \mathrm{g} / \mathrm{mL}$; number of detections $(\mathrm{n})=31)$, whereas common carp had the lowest Vtg concentrations $(1.44 \mu \mathrm{g} / \mathrm{mL}$; $\mathrm{n}=29)$ among species. South Fork Crow River had the greatest average Vtg concentration $(42.8 \mu \mathrm{g} / \mathrm{mL} ; \mathrm{n}=76)$ and the Redwood River had the lowest average Vtg concentration (3.65 $\mu \mathrm{g} / \mathrm{mL} ; \mathrm{n}=85)$ among all streams with all species and sites combined. Fish from upstream sites had lower average
Vtg concentrations $(6.02 \mu \mathrm{g} / \mathrm{mL} ; \mathrm{n}=78)$ than fish from downstream locations $(22.5 \mu \mathrm{g} / \mathrm{mL} ; \mathrm{n}=158)$. However, the number of male fish with Vtg detections was greater at upstream (62 percent) than downstream (55 percent) locations.

GSIs were calculated for white sucker, common carp, and redhorse species. GSI values ranged from 0.06 to 8.82 among all male fish analyzed; the average was 3.62. For all species and sites combined, fish from the Redwood River $(5.20 ; n=12)$ had the greatest average GSI values followed by fish from the South Fork Crow River (4.00; $\mathrm{n}=59$ ) and the Grindstone River $(0.88 ; n=15)$. Overall, male common carp had the greatest average GSI $(5.16 ; n=56)$ among species. Hepatosomatic index values ranged from 0.12 to 7.1 among all male fish collected. Average hepatosomatic index values were greatest at the Redwood River with all species and sites combined (2.13; $\mathrm{n}=13$ ) and least at the Grindstone River (1.40; $\mathrm{n}=15)$.

Testicular histological analyses (occurrence of intersex, spermatazoa abundance, seminiferous tubule degradation, spermatogonia abundance, and Sertoli and Leydig cell hypertrophy) were performed on the 199 of the 508 male fish that were determined to be spawning. The remaining males were either in an advanced stage of spawning or immature and thus histological analyses were not possible. Liver histological analysis and liver adipocyte abundance were performed on 500 male fish (livers were not recovered from the remaining 8 males). Intersex was observed in only one male fathead minnow at the upstream Grindstone River site (GRIND-US1) (appendix 10).

Spermatogonia abundance scores ranged from 0 (absent) to 4 (abundant) among all male fish collected. Fish from Redwood River had the greatest $(2.4 ; n=26)$ average spermatogonia abundance score, and Grindstone River had the lowest $(1.2 ; n=19)$ average score with all sites and species combined. Common carp had the greatest $(3.6 ; n=56)$ average spermatogonia abundance score, and creek chubs had the least $(0.7 ; n=2)$ average spermatogonia abundance score. With all species and sites combined, fish from downstream sites had a greater average spermatagonia score $(2.2 ; n=150)$ than those from upstream locations $(1.8 ; \mathrm{n}=49)$.

Seminiferous tubule degradation scores ranged from 0 (absent) to 4 (abundant) among all male fish. With all species and sites combined, fish from the South Fork Crow River had the greatest $(1.2 ; n=154)$ average seminiferous tubule degradation score, and those from Redwood River had the least (0.7; $\mathrm{n}=26)$ average score. White sucker $(1.3 ; \mathrm{n}=4)$ and redhorse $(1.3 ; n=4)$ had the greatest average degradation scores among all species sampled. Creek chub had the lowest $(0.7 ; n=2)$ average degradation scores among species sampled. With all species and sites combined, fish from upstream sites had a greater average seminiferous tubule degradation $(1.2 ; n=49)$ than those from downstream sites $(1.1 ; n=150)$.

Spermatazoa abundance scores ranged from 0 (none present) to 4 (abundant). The South Fork Crow River had the greatest $(2.5 ; n=154)$ average spermatozoa abundance, and the Redwood River had the least $(1.9 ; n=26)$ average abundance among the three streams with all species and sites combined. 
Common shiners $(2.9 ; \mathrm{n}=36)$ and fathead minnows $(2.9 ; \mathrm{n}=97)$ had the greatest average spermatazoa abundance among all species, and the common carp $(1.3 ; \mathrm{n}=56)$ and redhorse spp. $(0 ; n=4)$ had the least average spermatozoa abundance among species sampled.

Sertoli and Leydig cell hypertrophy scores ranged from 0 (absent) to 4 (abundant) among all fish measured. White sucker had the greatest $(4.0 ; n=4)$ average score among all species, and fathead minnows had the least $(1.0, n=97)$ average score. Fish from the Grindstone River had the greatest (1.9; $\mathrm{n}=19$ ) average Sertoli and Leydig cell hypertrophy score, and those from the South Fork Crow River had the least (1.1; $\mathrm{n}=154$ ) average score among all streams with all species and sites combined.

Liver adipocyte abundance scores ranged from 0 (absent) to 4 (abundant) among all fish. Fish from the South Fork Crow River had the greatest $(0.6 ; n=197)$ average liver adipocyte abundance, and those from the Grindstone River had the least $(0.3 ; \mathrm{n}=131)$ average abundance with all species and sites combined. Creek chub had the greatest $(0.8 ; n=44)$ average score among all species, and white sucker had the lowest $(0.1$; $\mathrm{n}=53$ ) average score.

\section{Summary}

This report presents the study design and environmental data from an integrated biological and chemical investigation of the occurrence of, distribution of, and biological response to alkylphenols and other endocrine-active chemicals in three small streams in Minnesota (South Fork Crow River, Redwood River, and Grindstone River) receiving wastewater. This study was a cooperative effort among the U.S. Geological Survey (USGS), Minnesota Pollution Control Agency, and St. Cloud State University. A description of the sites; sample collection and laboratory analyses; environmental conditions during sampling; and the water chemistry, bed-sediment chemistry, and biological data are contained in this report.

USGS staff measured streamflow, dissolved oxygen, $\mathrm{pH}$, specific conductance, and water temperature, and collected water and quality-assurance samples during six sampling events in each of three studied streams during February through September 2007. Water and bed sediment were collected upstream from and at two successive points downstream from discharge of wastewater-treatment plant (WWTP) effluent and from the treated effluent six times from February through September 2007.

Water and bed-sediment samples were analyzed for endocrine-active chemicals (including alkylphenols, alkylphenol polyethoxylates, and nonylphenolethoxy carboxylates) at two USGS laboratories. Water samples were also analyzed for major ions, nutrients, and organic carbon. In addition, as part of an intensive time-series investigation, the USGS collected daily water samples for 8 weeks at the Redwood River near Marshall, Minn., to analyze them for atrazine and total alkylphenols.

To determine biological responses, St. Cloud State University staff collected multiple fish species at the same locations as the water and bed sediments were collected and at one additional site near the WWTP effluent discharge location.

Streamflow was greatest in the Redwood River followed by the South Fork Crow River and the Grindstone River and was least during winter under ice cover and greatest March and April during snowmelt runoff. The percentage of streamflow contributed by WWTP effluent (ranging from less than 1 to 79 percent) was greatest at the South Fork Crow River and least at the Grindstone River.

Calcium, magnesium, potassium, sodium, and chloride concentrations were greater at the upstream sites on the South Fork Crow and Redwood Rivers than the Grindstone River. Average specific conductance in stream samples was greatest at the Redwood River (1,260 microsiemens per centimeter $(\mu \mathrm{S} / \mathrm{cm}))$, followed by the South Fork Crow River $(799 \mu \mathrm{S} / \mathrm{cm})$ and the Grindstone River $(189 \mu \mathrm{S} / \mathrm{cm})$. Specific conductance values were greater in WWTP effluent samples and at stream sites downstream from WWTP effluent discharge locations than at the upstream sites.

Total nitrogen and total phosphorus concentrations were greatest in the South Fork Crow River followed by the Redwood River, and then the Grindstone River. Total nitrogen (nitrate + nitrite + ammonia + organic $\mathrm{N}$ ) and total phosphorus concentrations increased downstream from WWTP effluent discharges on each river.

Average total organic carbon (TOC) concentrations ranged from 3.9 to 22.7 milligrams per liter $(\mathrm{mg} / \mathrm{L})$ and were generally lower in the Redwood River than in the South Fork Crow or Grindstone Rivers. TOC concentrations in WWTP effluent ranged from 5.8 to $11.9 \mathrm{mg} / \mathrm{L}$ among all WWTPs, and Hinckley effluent samples tended to have the lowest TOC concentrations among WWTPs.

The most commonly detected organic chemicals (in greater than 40 percent of the environmental samples) analyzed at the USGS National Research Program Laboratory (USGS-NRP) were 2,6-di-tert-butyl-1,4-benzoquinone, 2,6-di-tert-butyl-4-methylphenol, 3-beta-coprostanol, 4-methylphenol, 4-nonylphenol (NP), 4-tert-octylphenol (TOP), bisphenol A, cholesterol, ethylenediaminetetraacetic acid (EDTA), and triclosan.

EDTA concentrations, which ranged from 0.7 to 960 micrograms per liter $(\mu \mathrm{g} / \mathrm{L})$ among all samples, were greater in WWTP effluent samples than in stream samples and were detected at sites downstream from WWTP effluent discharge in all three streams. EDTA was detected at sites downstream from WWTP effluent discharge in each stream at concentrations ranging from 0.7 to $62 \mu \mathrm{g} / \mathrm{L}$.

The chemicals 4-nonylphenolmonoethoxycarboxylate (NP1EC), 4-nonylphenoldiethoxycarboxylate (NP2EC), and 4-nonylphenoltriethoxycarboxylate (NP3EC) were detected in study samples, and NP1EC and NP2EC were the dominant NPECs detected. The sum of NP1EC through NP3EC con- 
centrations (ranging from 5.1 to $260 \mu \mathrm{g} / \mathrm{L}$ among all samples) were detected in effluent samples from all three WWTPs. NPECs were detected at downstream sites on the Redwood and South Fork Crow Rivers; NPECs were not detected at the downstream sites on the Grindstone River.

Alkylphenols and alkylphenol ethoxylates were detected in upstream, effluent, and downstream samples in each stream. NP and TOP were detected in upstream, effluent, and downstream samples in each stream. NP was detected in 49 percent of environmental samples. Excluding nondetections, concentrations of NP ranged from 100 to 880 nanograms per liter (ng/L) among all samples. The single greatest NP concentration in a stream sample was measured at the second downstream site on the Grindstone River. TOP concentrations in effluent samples were greatest at the Hinckley WWTP, where the maximum TOP concentration of $110 \mathrm{ng} / \mathrm{L}$ was measured. NP1EO and NP2EO were detected at upstream sites, effluent sites, and at downstream sites along each stream and were detected more commonly at the Redwood River and Grindstone River sites than at the South Fork Crow River sites. Concentrations and number of detections of NP1EO and NP2EO in effluent samples were greater at Marshall and Hinckley than at Hutchinson.

Bisphenol A and triclosan were detected in upstream, effluent, and downstream samples in all three streams. Bisphenol A concentrations ranged from 4.87 to $137.40 \mathrm{ng} / \mathrm{L}$ among all samples. Triclosan concentrations ranged from 7.04 to $150 \mathrm{ng} / \mathrm{L}$ among all samples.

The most commonly detected wastewater indicator chemicals analyzed at the USGS National Water Quality Laboratory (NWQL) using analytical schedule 4433 during two sampling periods were 3,4-dichlorophenyl isocyanate, acetyl-hexamethyltetrahydronaphthalene (AHTN), benzophenone, cholesterol, hexahydrohexamethyl-cyclopenta-benzopyran (HHCB), N,Ndiethyl-meta-toluamide (DEET), and tri(dichloroisopropyl) phosphate. Some chemicals were detected in all effluent samples (1,4-dichlorobenzene, benzophenone, HHCB, DEET, triethyl citrate, tri(2-chloroethyl) phosphate, and tri(dichloroisopropyl) phosphate), and others were detected in most (five out of six) WWTP effluent samples (3-methyl-1H-indole, 4-methylphenol, and AHTN). Most of these chemicals also were detected in corresponding samples at downstream sites.

As part of the time-series data collection, atrazine was detected in 60 of the 62 samples, and concentrations ranged from 1.93-46.41 ng/L among samples. Total APs were detected in 5 of the 62 samples collected at concentrations ranging from $50-65 \mathrm{ng} / \mathrm{L}$.

The most commonly detected chemicals in bed-sediment samples were 2,6-di-tert-butyl-1,4-benzoquinone, 3-betacoprostanol, 4-methylphenol, and cholesterol. NP and NP1EO were detected in bed sediment only at sites downstream from WWTP discharge locations. NP was detected at downstream locations in all three streams at concentrations ranging from 24-260 nanograms per gram (ng/g) and NP1EO was detected at the two downstream locations on the Grindstone River at concentrations of 15 and $17 \mathrm{ng} / \mathrm{g}$.
St. Cloud State University targeted six species of fish for collection at all water and bed-sediment sampling sites and at an additional site near the WWTP effluent discharge in each stream. During this study, 508 male and 397 female fish were collected. Weights and lengths were measured and condition factors were calculated for all fish. Female fish were collected incidentally and some biological characterizations were completed, but the focus of the study was on male fish responses. Complete plasma and histological analysis was attempted for all male fish collected, but it was not possible to perform all analyses on all fish owing to fish size, reproductive stage, and availability of tissue or plasma for characterization.

Vitellogenin (Vtg) was detected in 57 percent of the 415 male fish from which sufficient blood was drawn; Vtg concentrations ranged from an estimated value of 0.1 to $330 \mu \mathrm{g} / \mathrm{mL}$ (average of $17.1 \mu \mathrm{g} / \mathrm{mL}$ for the 237 male fish with detections). Vtg analyses were also done on 138 female fish, and Vtg was detected in 71 percent of them; concentrations ranged from 0.059 to $54,300 \mu \mathrm{g} / \mathrm{mL}$ (average $1,538.4 \mu \mathrm{g} / \mathrm{mL}$ ).

Gonadosomatic indexes (GSIs) for white sucker, common carp, and redhorse species ranged from 0.06 to 8.82 among all male fish collected; the average was 3.62. For all species and sites combined, fish from the Redwood River (5.20) had the greatest average GSI values followed by those from the South Fork Crow River (4.00) and the Grindstone River (0.88).

Testicular histological analyses (occurrence of intersex, spermatazoa abundance, seminiferous tubule degradation, spermatogonia abundance, Sertoli and Leydig cell hypertrophy, and intersex occurrence) were performed on the 199 of the 508 male fish that were determined to be spawning. Liver histological analysis was performed on 500 male fish. Male fish from the Redwood River had the greatest average spermatogonia abundance score (2.4), and Grindstone River had the lowest (1.2) average score with all sites and species combined. The Grindstone River had the greatest (1.2) average seminiferous tubule degradation score, and Redwood River had the least (0.7) average score. The South Fork Crow River had the greatest (2.5) average spermatozoa abundance and the Grindstone River had the least (1.9) average spermatozoa abundance among the three streams. Fish from the South Fork Crow River had the greatest (0.6) average liver adipocyte abundance, and those from the Grindstone River had the least (0.3) average abundance. Intersex (occurrence of oocytes in testes tissue) was observed in only one fathead minnow from an upstream site on the Grindstone River.

\section{References Cited}

Ahel, Marijan, Giger, Walter, and Koch, Markus, 1994a, Behaviour of alkylphenol polyethoxylate surfactants in the aquatic environment. I. Occurrence and transformation in sewage treatment: Water Research, v. 28, no. 5, p. 1,131-1,142. 
Ahel, Marijan, Giger, Walter, and Schaffner, Christian, 1994b, Behaviour of alkylphenol polyethoxylate surfactants in the aquatic environment. II. Occurrence and transformation in rivers: Water Research, v. 28, no. 5, p. 1,143-1,152.

Allen, Yvonne, Scott, A.P., Matthiessen, Peter, Haworth, Sarah, Thain, J.E., and Feist, Steve, 1999, Survey of estrogenic activity in United Kingdom estuarine and coastal waters and its effects on gonadal development of the flounder Platichthys flesus: Environmental Toxicology and Chemistry, v. 18 , no. 8 , p. 1,791-1,800.

Ankley, Gary, Francis, E., Gray, E., Kavlock, R., McMaster, S., Reese, D., Sayles, G., Sergeant, A., and Vallero, D., 1998, Research plan for endocrine disruptors: Research Triangle Park, N.C., Office of Research and Development, U.S. Environmental Protection Agency, EPA600/R-98/087, 47 p.

Barber, L.B., II, Leenheer, J.A., Pereira, W.E., Noyes, T.I., Brown, G.K., Tabor, C.F., and Writer, J.H., 1996, Organic contamination of the Mississippi River from municipal and industrial wastewater: U.S. Geological Survey Circular 1133, p. 114-135.

Barber, L.B., Brown, G.K., and Zaugg, S.D., 2000, Potential endocrine disrupting organic chemicals in treated municipal wastewater and river water, chap. 7 of Keith, L.H., JonesLepp, T.L., and Needham, L.L., eds., Analysis of environmental endocrine disruptors: Washington, D.C., American Chemical Society, American Chemical Society Symposium Series 747, p. 97-123.

Barber, L.B., Leenheer, J.A., Noyes, T.I., and Stiles, E.A., 2001, Nature and transformation of dissolved organic matter in treatment wetlands: Environmental Science and Technology, v. 35, p. 4,805-4,816.

Barber, L.B., Furlong, E.T., Keefe, S.H., Brown, G.K., and Cahill, J.D., 2003, Natural and contaminant organic compounds in the Boulder Creek watershed, Colorado, during high-flow and low-flow conditions, 2000, chap. 5 of U.S. Geological Survey Water-Resources Investigations Report 2003-4045, p. 103-144.

Barber, L.B., Keefe, S.H., Brown, G.K., Taylor, H.E., Antweiler, R.C., Peart, D.B., Plowman, T.I., Roth, D.A., and Wass, R.D., 2003, Organic and trace element contaminants in water biota, sediment, and semipermeable membrane devices at the Tres Rios Treatment Wetlands, Phoenix, Arizona: U.S. Geological Survey Water-Resources Investigations Report 03-4129, 89 p.

Barber, L.B., Lee, K.E., Swackhammer, D.L., and Schoenfuss, H.L., 2007, Reproductive responses of male fathead minnows exposed to wastewater treatment plant effluent, effluent treated with XAD8 resin, and an environmentally relevant mixture of alkylphenol compounds: Aquatic Toxicology, v. 82, p. 36-46.
Barnes, K.K., Kolpin, D.W., Meyer, M.T., Thurman, E.M., Furlong, E.T., Zaugg, S.D., and Barber, L.B., 2002, Waterquality data for pharmaceuticals, hormones, and other organic wastewater contaminants in U.S. streams, 19992000: U.S. Geological Survey Open-File Report 02-94.

Becker, G.C., 1983, Fishes of Wisconsin: Madison, Wisc., University of Wisconsin Press, p. 653-677.

Bistodeau, T.J., Barber, L.B., Bartell, S.E., Cediel, R.A., Grove, K.J., Klaustermeier, Jacob, Woodard, J.C., Lee, K.E., and Schoenfuss, H.L., 2006, Larval exposure to environmentally relevant mixtures of alkylphenolethoxylates reduces reproductive competence in male fathead minnows: Aquatic Toxicology, v. 79, p. 268-277.

Burkhardt, M.R., Zaugg, S.D., Smith, S.G., and ReVello, R.C., 2006, Determination of wastewater compounds in sediment and soil by pressurized solvent extraction, solid-phase extraction, and capillary-column gas chromatography/ mass spectrometry: U.S. Geological Survey Techniques and Methods, book 5, chap. B2, 90 p.

Childress, C.J.O., Foreman, W.T., Connor, B.F., and Maloney, T.J., 1999, New reporting procedures based on longterm method detection levels and some considerations for interpretations of water-quality data provided by the U.S. Geological Survey National Water Quality Laboratory: U.S. Geological Survey Open-File Report 99-193, 19 p.

Copes, F.C., 1978, Ecology of the creek chub Semotilus atromaculatus (Mitchill) in northern waters: University of Wisconsin-Stevens Point, Museum of Natural History Fauna and Flora, Wisconsin Report 12, p. 1-21.

Desbrow, C., Routledge, E.J., Brighty, G.C., Sumpter, J.P., and Waldock, M., 1998, Identification of estrogenic chemicals in STW effluent. 1. Chemical fractionation and in vitro biological screening: Environmental Science and Technology, v. 32 , p. $1,549-1,558$.

EC-BKH, 2000, Towards the establishment of a priority list of substances for further evaluation of their role in endocrine disruption (final report): Delft, The Netherlands, BKH Consulting Engineers, European Commission DG ENV, accessed July 14, 2008, at http://ec.europa.eu/environment/ docum/01262_en.htm

Eddy, S., and Underhill, J.C., 1974, Northern fishes, with special reference to the Upper Mississippi Valley ( $3 \mathrm{~d}$ ed.): Minneapolis, University of Minnesota Press, p. 218-220, 285-288.

Edwards, T.K., and Glysson, G.D., 1988, Field methods for measurement of fluvial sediment: U.S. Geological Survey Open-File Report 86-531, 118 p. 
Fishman, M.J., 1993, Methods of analysis by the U.S. Geological Survey National Water Quality Laboratory-Determination of inorganic and organic constituents in water and fluvial sediments: U.S. Geological Survey Open-File Report 93-125, $217 \mathrm{p}$.

Fishman, J.J., and Friedman, L.C., eds., 1989, Methods for the determination of inorganic substances in water and fluvial sediments: U.S. Geological Survey Techniques of Water Resources Investigations, book 5, chap. A1, 709 p.

Fishman, M.J., Raese, J.W., Gerlitz, C.N., and Husband, R.A., 1994, U.S. Geological Survey approved inorganic and organic methods for the analysis of water and fluvial sediment, 1954-94: U.S. Geological Survey Open-File Report 94-351, 55 p.

Folmar, L.C., Denslow, N.D., Kroll, K., Orlando, E.F., Enblom, J., Marcino, J., Metcalfe, C., and Guillette, L.J., Jr., 2001, Altered serum sex steroids and vitellogenin induction in walleye (Stizostedion vitreum) collected near a metropolitan sewage treatment plant: Archives of Environmental Contamination Toxicology, v. 40, p. 392-398.

Folmar, L.C., Denslow, N.D., Rao, V., Chow, M., Crain, D.A., Enblom, J., Marcino, J., and Guillette, L.J., Jr., 1996, Vitellogenin induction and reduced serum testosterone concentrations in feral male carp (Cyprinus carpio) captured near a major metropolitan sewage treatment plant: Environmental Health Perspectives, v. 104, p. 1,096-1,101.

Funk, J.L., 1955, Movement of stream fishes in Missouri: Transactions of the American Fisheries Society, v. 85, no. 1, p. 39-57.

Gabe, Manfred, 1976, Histological techniques: New York, Springer-Verlag, $106 \mathrm{p}$.

Giger, Walter, Ahel, M., Koch, M., Laubscher, H.U., Schaffner, C., and Schneider, J., 1987, Behaviour of alkylphenol polyethoxylate surfactants and of nitrilotriacetate in sewage treatment: Water, Science and Technology, v. 19, p. 449-460.

Giger, Walter, Stephanou, E., and Schaffner, C., 1981, Persistent organic chemicals in sewage effluents. I. Identifications of nonylphenols and nonylphenolethoxylates by glass capillary gas chromatography/mass spectrometry: Chemosphere, v. 10, p. 1,253-1,263.

Glassmeyer, S.T., Furlong, E.T., Kolpin, D.W., Cahill, J.D., Zaugg, A.D., Werner, S.L., Meyer, M.T., and Kryak, D.D., 2005, Transport of chemical and microbial compounds from known wastewater discharges - Potential for use as indicators of human fecal contamination: Environmental Science and Technology, v. 39, no. 14, p. 5,157-5,169.
Global Water Research Coalition, 2003, Endocrine disrupting compounds, priority list of EDCs, September 2003: London, Global Water Research Coalition, 29 p.

Hackney, P.A., Tatum, W.M., and Spencer, S.L., 1968, Life history study of the river redhorse, M. carinatum (Cope) in the Cahaba River, AL, with notes on the management of the species as a sport fish: Southeast Association of Game Fish Commissioners, Proceedings, v. 21, p. 324-332.

Harries, J.E., Runnalls, T., Hill, E., Harris, C.A., Maddix, S., Sumpter, J.P., and Tyler, C.R., 2000, Development of a reproductive performance test for endocrine disrupting chemicals using pair-breeding fathead minnows (Pimephales promelas): Environmental Science and Technology, v. 34, p. 3,003-3,011.

Hem, J.D., 1985, Study and interpretation of the chemical characteristics of natural water ( $3 \mathrm{~d}$ ed.): U.S. Geological Survey Water-Supply Paper 2254, 263 p.

Institute of Environmental Health, 2005, Chemicals purported to be endocrine disrupters-A compilation of published lists: Leicester, U.K., MRC Institute for Environment and Health, Web Report W20, accessed July 13, 2008, at http:// www.silsoe.cranfield.ac.uk/ieh/pdf/w20.pdf

Jahns, N.D., 2008, Endocrine disruption of fishes in three point and non-point source influenced tributaries of the Mississippi River: St. Cloud, Minn., St. Cloud State University, Masters thesis, variously paged.

Jobling, Susan, Sheahan, D., Osborne, J.A., Matthiessen, P., and Sumpter, J.P., 1996, Inhibition of testicular growth in rainbow trout (Oncorhynchus mykiss) exposed to estrogenic alkylphenolic chemicals: Environmental Toxicology and Chemistry, v. 15, p. 194-202.

Jobling, Susan, and Sumpter, J.P., 1993, Detergent components in sewage effluent are weakly oestrogenic to fish-An in vitro study using rainbow trout (Oncorhynchus mykiss) hepatocytes: Aquatic Toxicology, v. 27, p. 361-372.

Johnson, A.C., and Sumpter, J.P., 2001, Removal of endocrinedisrupting chemicals in activated sludge treatment works: Environmental Science and Technology, v. 35, p. 4,697-4,703.

Kime, D.E., 1998, Endocrine disruption in fish: Boston, Kluwer Academic Publishers, 396 p.

Kolpin, D.W., Furlong, E.T., Meyer, M.T., Thurman, E.M., Zaugg, S.D., Barber, L.B., and Buxton, H.T., 2002, Pharmaceuticals, hormones, and other organic wastewater contaminants in U.S. streams, 1999-2000-A national reconnaissance: Environmental Science and Technology, v. 36, p. 1,202-1,211. 
Korner, Oliver, Vermeirssen, L.M., and Burkhardt-Holm, P., 2005 , Intersex in feral brown trout from Swiss midland rivers: Journal of Fish Biology, v. 67, p. 1,734-1,740.

Lee, K.E., Blazer, V.S., Denslow, N.D., Goldstein, R.M., and Talmage, P.J., 2000, Use of biological characteristics of common carp (Cyprinus carpio) to indicate exposure to hormonally active agents in selected Minnesota streams, 1999: U.S. Geological Survey Water-Resources Investigations Report 00-4202, 47 p.

Lee, K.E., Barber, L.B., Furlong, E.T., Cahill, J.D., Kolpin, D.W., Meyer, M.T., and Zaugg, S.D., 2004, Presence and distribution of organic wastewater compounds in wastewater, surface, ground, and drinking waters, Minnesota, 2000-02: U.S. Geological Survey Scientific Investigations Report 2004-5138, 47 p.

Lee, K.E., and Blazer, V., 2005, Endocrine disruption in Minnesota fish-A comparison of fish species Cyprinus carpio and Micropterus: Madison, Wisc., Society of Environmental Toxicology and Chemistry, v. 136, p. 381-388.

Lee, K.E., Yaeger, C.S., Jahns, N.D., and Schoenfuss, H.L., 2008, Occurrence of endocrine active compounds and biological responses in the Mississippi River-Study design and data, June-August 2006: U.S. Geological Survey Data Series 368, 27 p., online at http://pubs.usgs.gov/ds/368/.

Leenheer, J.A., Rostad, C.E., Barber, L.B., Schroeder, R.A., Anders, R., and Davisson, M.L., 2001, Nature and chlorine reactivity of organic constituents from reclaimed water in groundwater, Los Angeles County, California: Environmental Science and Technology, v. 35, p. 3,869-3,876.

Martinovic, Dalma, Denny, J.S., Schmieder, P.K., Ankley, G.T., and Sorensen, P.W., 2008, Temporal variation in the estrogenicity of a sewage treatment plant effluent and its biological significance: Environmental Science and Technology, v. 42, p. 3,421-3,427.

Miles-Richardson, S.R., Kramer, V.J., Fitzgerald, S.D., Render, J.A., Yamini, B., Barbee, S.J., and Giesy, J.P., 1999, Effects of waterborne exposure of $17 \beta$-estradiol on secondary sex characteristics and gonads of fathead minnows (Pimephales promelas): Aquatic Toxicology, v. 47, p. 129-145.

Miller, R.J., 1964, Behavior and ecology of some North American cyprinid fishes: American Midland Naturalist, v. 72, p. 313-357.

Minnesota Pollution Control Agency, 2000, Upper Mississippi River Basin Information Document, St. Paul, Minn., Minnesota Pollution Control Agency: accessed June 10, 2008, at http://www.pca.state.mn.us/water/basins/uppermiss/biduppermiss.pdf
Minnesota Pollution Control Agency, 2004, Public Notice of Intent to Reissue National Pollutant Discharge Elimination System and State Disposal System Permit for the Marshall Wastewater Treatment Facility, MN0022179: accessed July 8, 2008, at http://www.pca.state.mn.us/news/data/bdc.cfm?n otice $I D=262524 \&$ blobID $=6268 \&$ docType $I D=4$

Minnnesota Pollution Control Agency, 2005, Public Notice of Intent to Reissue National Pollutant Discharge Elimination System and State Disposal System Permit for the Hinckley Wastewater Treatment Facility, MN0023701: accessed July 8, 2008, at http://www.pca.state.mn.us/news/data/bdc.cfm?n otice $I D=266304 \&$ blobID $=9228 \&$ docType $I D=4$

Minnesota Pollution Control Agency, 2007, Public Notice of Intent to Reissue National Pollutant Discharge Elimination System and State Disposal System Permit for the Hutchinson Wastewater Treatment Facility, MN0055832: accessed July 8, 2008, at http://proteus.pca.state.mn.us/news/data/ bdc.cfm $?$ notice $I D=275052 \&$ blobID $=17610 \&$ docType $I D=4$

Minnesota Pollution Control Agency, 2008, Minnesota River Basin, Redwood River watershed basin report: accessed July 8, 2008, at http://www.pca.state.mn.us/water/basins/ mnriver/watershed-redwood.pdf

Morlock, S.E., Nguyen, H.T., and Ross, J.H., 2002, Feasibility of acoustic doppler velocity meters for the production of discharge records from U.S. Geological Survey streamflow gaging stations: U.S. Geological Survey Water-Resources Investigations Report 01-4157, 59 p.

National Research Council, 1999, Hormonally active agents in the environment: Washington, D.C., National Academy Press, 429 p.

Naylor, C.G., Mieure, J.P., Adams, W.J., Weeks, J.A., Castaldi, F.J., Ogle, L.D., Romano, R.R., 1992, Alkylphenol ethoxylates in the environment: Journal of American Oil Chemical Society, v. 69, p. 675-703.

Nishihara, T., Nishikawa, J., Hori, S., Saito, K., Kanayama, T., Imagawa, M., Takatori, S., Kitagawa, K., Dakeyama, F., and Utsumi, H., 2000, Oestrogenic activities of 517 chemicals by yeast two-hybrid assay: Journal of Health Sciences, v. 46, p. 282-298.

Olson, D.E., and Scidmore, W.J., 1963, Homing tendency of spawning white suckers in Many Point Lake, Minnesota: Transactions of the American Fisheries Society, v. 92, no. 1, p. 13-16.

Patterson, C.J., 1997, Contributions to the Quaternary geology of southwestern Minnesota: Minnesota Geological Survey, University of Minnesota Report of Investigations 47, 78 p. 
Patton, C.J., and Truitt, E.P., 1992, Methods of analysis by the U.S. Geological Survey National Water Quality Laboratory-Determination of total phosphorus by a Kjeldahl digestion method and an automated colorimetric finish that includes dialysis: U.S. Geological Survey Open-File Report 92-146, 39 p.

Rantz, S.E., and others, 1982a, Measurement and computation of streamflow. 1. Measurement of stage and discharge: U.S. Geological Survey Water-Supply Paper 2175, p. 1-284.

Rantz, S.E., and others, 1982b, Measurement and computation of streamflow. 2. Computation of discharge: U.S. Geological Survey Water-Supply Paper 2175, p. 285-631.

Schaffner, C., and Giger, W., 1984, Determination of nitrilotriacetic acid in water by high-resolution gas chromatography: Journal of Chromatography, v. 312, p. 413-421.

Schlosser, I.J., 1995, Dispersal, boundary processes, and trophic-level interactions in streams adjacent to beaver ponds: Ecology, v. 76 , no. 3, p. 908-925.

Schoenfuss, H.L., Bartell, S.E., Bistodeau, T.B., Cediel, R.A., Grove, K.J., Zintek, L., Lee, K.E., and Barber, L.B., 2008, Impariment of the reproductive potential of male fathead minnows by environmentally relevant exposures to 4-nonylphenol: Aquatic Toxicology, v. 86, p. 91-98.

Talmage, S.S., 1994, Environmental and human safety of major surfactants-Alcohol ethoxylates and alkylphenol ethoxylates: Boca Raton, Fla., Lewis Publishers, 374 p.

Terasaki, M., Shiraishi, F., Nishikawa, T., Edmonds, J.S., Morita, M., and Makino, M., 2005, Estrogenic activity of impurities in industrial grade bisphenol A: Environmental Science and Technology, v. 39, no. 10, p. 3,703-3,707.

Ternes, T.A., 1998, Occurrence of drugs in German sewage treatment plants and rivers: Water Research, v. 32, no. 11, p. $3,245-3,260$.

Ternes, T.A., Stumpf, M., Mueller, J., Haberer, K., Wilken, R.D., and Servos, M., 1999, Behavior and occurrence of estrogens in municipal sewage treatment plants. I. Investigations in Germany, Canada, and Brazil: Science of the Total Environment, v. 225, p. 81-90.

Thorpe, K.L., Hutchinson, T.H., Hetheridge, M.J., Scholze, M., Sumpter, J.P., and Tyler, C.R., 2001, Assessing the biological potency of binary mixtures of environmental estrogens using vitellogenin induction in juvenile rainbow trout (Oncorhynchus mykiss): Environmental Science and Technology, v. 35, p. 2,476-2,481.

Thurman, E.M., 1985, Organic geochemistry of natural waters: Boston, Martinus Nijhoff/Dr W. Junk Publishers, $497 \mathrm{p}$.
Timme, P.J., 1995, National Water Quality Laboratory 1995 services catalog: U.S. Geological Survey Open-File Report 95-352, p. 92.

Tremblay, Louis, and Van der Kraak, Glen, 1998, Use of a series of homologous in vitro and in vivo assays to evaluate the endocrine modulating actions of $b$-sitosterol in rainbow trout: Aquatic Toxicology, v. 43, p. 149-162.

University of Minnesota, 2008, Historical climate data retrieval: accessed May 2008, at http://climate.umn.edu/doc/ historical.htm

U.S. Department of Agriculture Natural Resources Conservation Service, 2006, Land resource regions and major land resource areas of the United States, the Caribbean, and the Pacific Basin: U.S. Department of Agriculture Handbook 296.

U.S. Environmental Protection Agency, 1993, Methods for the determination of inorganic substances in environmental samples-Environmental Monitoring Systems Laboratory, Cincinnati, Ohio: U.S. Environmental Protection Agency EPA/600/R-93/100, variously paginated.

U.S. Environmental Protection Agency, 2002, Guidelines establishing test procedures for the analysis of pollutants (Part 136, Appendix B). Definition and procedure for the determination of the method detection limit-Revision 1.11: U.S. Code of Federal Regulations, Title 40, revised July 1, 2002, p. 635-638.

U.S. Environmental Protection Agency, 2005a, Ambient aquatic life water quality criteria for nonylphenol (final): Washington, D.C., EPA/822-R-05-005.

U.S. Environmental Protection Agency, 2005b, EPI suite website: accessed January 12, 2006, at http://www.epa.gov/oppt/ exposure/pubs/episuite.htm

U.S. Environmental Protection Agency, 2006a, Inert reassessments-Four exemptions from the requirement of tolerances for nonylphenol ethoxylates: Office of Prevention Pesticides and Toxic Substances Action Memorandum, July 31, 2006, accessed June 20, 2008, at http://www.epa.gov/opprd001/ inerts/nonylphenol.pdf

U.S. Environmental Protection Agency, 2006b, Histopathology guidelines for the fathead minnow (Pimephales promelas) 21-day reproduction assay, July 6, 2006, accessed June 20, 2008, at http://www.epa.gov/endo/pubs/att- $h_{-}$ histopathologyguidlines_fhm.pdf

U.S. Geological Survey, 2003, National field manual for the collection of water-quality data: Techniques of WaterResources Investigations, book 9, available at http://water. usgs.gov/owq/FieldManual/, variously paged. 
Vajda, A.M., Barber, L.B., Gray, J.L., Lopez, E.M., Woodling, J.D., and Norris, D.O., 2008, Reproductive disruption in fish downstream from an estrogenic wastewater effluent: Environmental Science and Technology, v. 42, p. 3,407-3,414.

Versonnen, B.J., Arijs, K., Verslycke, T., Lema, W., and Janssen, C.R., 2003, In vitro and in vivo estrogenicity and toxicity of o-, m-, and p-dichlorobenzene: Environmental Toxicology and Chemistry, v. 22, no. 2, p. 329-335.

Waters, T.F., 1977, The streams and rivers of Minnesota: Minneapolis, Minn., University of Minnesota Press, 373 p.

Weishaar, J.L., Aiken, G.R., Bergamaschi, B.A., Fram, M.S., Fujii, R., and Mopper, K., 2003, Evaluation of specific ultraviolet absorbance as an indicator of the chemical composition and reactivity of dissolved organic carbon: Environmental Science and Technology, v. 27, p. 4,702-4,708.

Zaugg, S.D., Smith, S.G., and Schroeder, M.P., 2006, Determination of wastewater compounds in whole water by continuous liquid-liquid extraction and capillary-column gas chromatography/mass spectrometry: U.S. Geological Survey Techniques and Methods, book 5, chap. B4, 30 p.

Zaugg, S.D., Smith, S.G., Schroeder, M.P., Barber, L.B., and Burkhardt, M.R., 2002, Methods of analysis by the U.S. Geological Survey National Water-Quality LaboratoryDetermination of wastewater compounds by polystyrenedivinylbenzene solid-phase extraction and capillary-column gas chromatography/mass spectrometry: U.S. Geological Survey Water-Resources Investigations Report 01-4186, $37 \mathrm{p}$.

Zhang, Fagen, Bartels, M.J., Brodeur, J.C., and Woodburn, K.B., 2004, Quantitative measurement of fathead minnow vitellogenin by liquid chromatography combined with tandem mass spectrometry using a signature peptide of vitellogenin: Environmental Toxicology and Chemistry, v. 23 , p. $1,408-1,415$. 


\section{Appendixes}

\section{Appendix 1. Concentrations of organic chemicals in water analyzed at U.S. Geological Survey National Research Program Laboratory}

The Excel spreadsheet Appendixl.xls contains concentration data for 41 chemicals and 8 surrogate standards for which water samples in this study were analyzed. Analyses of water samples collected from 13 sites during February-September 2007 and associated qualityassurance samples are contained in this spreadsheet.

\section{Appendix 2. Concentrations of organic chemicals in water samples analyzed using Laboratory Schedule $\mathbf{4 4 3 3}$ at the U.S. Geological Survey National Water Quality Laboratory.}

The Excel spreadsheet Appendix2.xls contains concentration data for 69 organic chemicals and 4 surrogate standards for which water samples in this study were analyzed. Analyses of water samples collected from 13 sites during May and August 2007 and associated qualityassurance samples are contained in this spreadsheet.

\section{Appendix 3. Concentrations of nutrients in water samples analyzed at U.S. Geological Survey National Water Quality Laboratory}

The Excel spreadsheet Appendix3.xls contains concentration data for six nutrients for which water samples in this study were analyzed. Analyses of water samples collected during February, March, and August 2007 from 13 sites and associated quality-assurance samples are contained in this spreadsheet.

\section{Appendix 4. Dissolved concentrations of major ions in water samples analyzed at the U.S. Geological Survey National Water Quality Laboratory}

The Excel spreadsheet Appendix4.xls contains concentration data for 10 major ions and residue on evaporation for which water samples in this study were analyzed. Analyses of water samples collected February through September 2007 from 13 sites and associated quality-assurance samples are contained in this spreadsheet.

\section{Appendix 5. Concentrations of organic chemicals analyzed in bed- sediment samples at the U.S. Geological Survey National Research Program Laboratory}

The Excel spreadsheet Appendix5.xls contains concentration data for 29 chemicals and 7 surrogate standards for which bed-sediment samples in this study were analyzed. Analyses of bed-sediment samples collected from 10 sites during June 2007 and associated qualityassurance samples are contained in this spreadsheet. 


\section{Appendix 6. Hydrologic characteristics of sampling sites}

The Excel spreadsheet Appendix6.xls contains hydrologic characteristics for the sampling sites. Hydrologic data contained in this spreadsheet include instantaneous discharge, drainage area, and percentage of flow composed of WWTP effluent.

\section{Appendix 7. Physical measurements of water quality}

The Excel spreadsheet Appendix7.xls contains data on physical measurements of water quality for samples collected February-October 2007. Physical measurements of dissolved oxygen, pH, specific conductance, and water temperature are contained in the spreadsheet.

\section{Appendix 8. Concentrations of total alkylphenols and atrazine in water analyzed at U.S. Geological Survey National Research Program Laboratory}

The Excel spreadsheet Appendix8.xls contains concentration data for total alkylphenols and atrazine in water samples collected daily from the Redwood River near Marshall, Minn., during April through July 2007.

\section{Appendix 9. Chemical characteristics of wastewater-treatment plant effluents studied}

The Excel spreadsheet Appendix9.xls contains monitoring data for three primary wastewatertreatment plants, one major industrial discharger, and wastewater-treatment plants upstream from sampling reaches on each stream. Data include year 2007 monthly or weekly average values for total suspended solids, carbonaceous biological oxygen demand, total nitrogen, and total phosphorus.

\section{Appendix 10. Characteristics of fish collected and analyzed by St. Cloud State University}

The Excel spreadsheet Appendix10.xls contains characteristics of six species of fish collected for this study. Analyses of fish samples collected from 13 sites during May through July 2007 are contained in this spreadsheet.

Publishing support provided by:

Rolla Publishing Service Center

For more information concerning this publication, contact:

Director, USGS Minnesota Water Science Center

2280 Woodale Drive

Mounds View, Minnesota 55112

(763) 783-3100

Or visit the Minnesota Water Science Center Web site at:

http://mn.water.usgs.gov 

